\title{
THE EXPLICIT SOLUTIONS OF LINEAR LEFT-INVARIANT SECOND ORDER STOCHASTIC EVOLUTION EQUATIONS ON THE 2D EUCLIDEAN MOTION GROUP
}

\author{
BY \\ REMCO DUITS (Department of Mathematics/Computer Science and Department of Biomedical \\ Engineering, Eindhoven University of Technology, Den Dolech 2, P.O. Box 513, 5600MB Eindhoven, \\ The Netherlands) \\ AND
}

MARKUS VAN ALMSICK (Department of Biomedical Engineering, Eindhoven University of Technology, Den Dolech 2, P.O. Box 513, 5600MB Eindhoven, The Netherlands)

\begin{abstract}
We provide the solutions of linear, left-invariant, second order stochastic evolution equations on the 2D Euclidean motion group. These solutions are given by group-convolution with the corresponding Green's functions which we derive in explicit form. A particular case coincides with the hitherto unsolved forward Kolmogorov equation of the so-called direction process, the exact solution of which is required in the field of image analysis for modeling the propagation of lines and contours. By approximating the left-invariant basis of the generators by left-invariant generators of a Heisenberg-type group, we derive simple, analytic approximations of the Green's functions. We provide the explicit connection and a comparison between these approximations and the exact solutions. Finally, we explain the connection between the exact solutions and previous numerical implementations, which we generalize to cope with all linear, left-invariant, second order stochastic evolution equations.
\end{abstract}

1. Introduction. Image analysis usually starts with the sampling of an image $f \in$ $\mathbb{L}_{2}\left(\mathbb{R}^{2}\right)$ by a function $\psi \in \mathbb{L}_{2}\left(\mathbb{R}^{2}\right)$ via $f \mapsto(\psi, f)_{\mathbb{L}_{2}\left(\mathbb{R}^{2}\right)}$. To probe an image at every location $\mathbf{x} \in \mathbb{R}^{2}$ and in every direction $e^{i \theta} \in \mathbb{T}$, one translates and rotates an anisotropic wavelet $\psi$ by means of a representation $g \mapsto \mathcal{U}_{g}$ of the Euclidean motion group $\mathcal{U}_{g} \psi(\mathbf{y})=\psi\left(R_{\theta}^{-1}(\mathbf{y}-\mathbf{x})\right), g=\left(\mathbf{x}, e^{i \theta}\right)$. The result of such an image sampling is a function $U_{f} \in \mathbb{L}_{2}(G)$ on the Euclidean motion group manifold $G=\mathbb{R}^{2} \rtimes \mathbb{T}$, which is

Received May 2, 2006.

2000 Mathematics Subject Classification. Primary 22E25, 37L05, 68U10; Secondary 34B30, 47D06.

Key words and phrases. Lie groups, stochastic evolution equations, image analysis, direction process, completion field.

The Netherlands Organization for Scientific Research is gratefully acknowledged for financial support. E-mail address: R.Duits@tue.nl

E-mail address: M.v.Almsick@tue.nl 

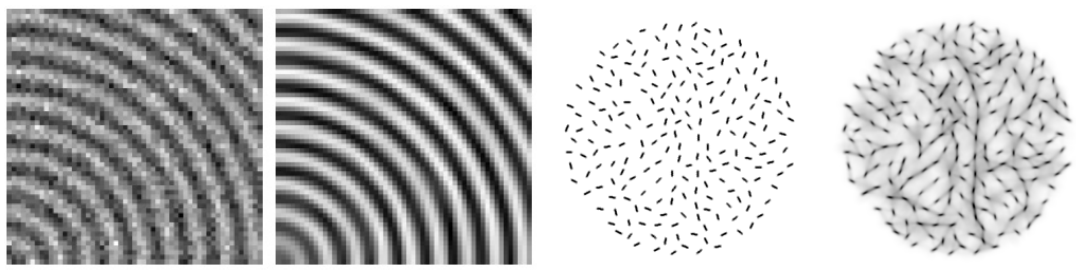

FIG. 1. Simple illustrations of automated contour enhancement (left) and contour completion (right) via left-invariant evolution equations on invertible orientation scores. For details on (medical) image analysis applications, see [27], [15], [47], [26] and [19].

given by $U_{f}(g)=\left(\mathcal{U}_{g} \psi, f\right)_{\mathbb{L}_{2}\left(\mathbb{R}^{2}\right)}$. Throughout this article we refer to function $U_{f}$ as the orientation score of image $f$.

The generation of orientation scores and the reconstruction of images thereof has been the subject of previous publications ([13], [15], [18], [35]). The subsequent Section 2 will provide a brief overview and an embedding in wavelet theory. For the remainder of this article we assume the orientation score as given and focus on operations on $U_{f}$ that are inspired by stochastic processes modeling the propagation of lines and contours.

As a class of left-invariant operators we consider in Section 3 all linear, second order, left-invariant evolution equations and their resolvents on $\mathbb{L}_{2}\left(\mathbb{R}^{2} \rtimes \mathbb{T}\right)$, which correspond to the forward Kolmogorov equations of left-invariant stochastic processes on the Euclidean motion group $\mathbb{R}^{2} \rtimes \mathbb{T}$ (i.e. the space of positions and orientations). We distinguish between two types of stochastic processes on the Euclidean motion group:

(1) stochastic processes for contour completion, including the direction process as proposed by Mumford [40],

(2) stochastic processes for contour enhancement, including the cortical model of the visual system for contour enhancement as proposed by Citti et al. [9].

See Figure 1. The mathematical difference between these stochastic processes is that the generator of their forward Kolmogorov equation of the first category, in contrast to the second category, contains a convection part that fills and bridges gaps in contours.

In this article we mainly consider linear stochastic processes for contour completion. For linear and non-linear stochastic processes for contour enhancement and their applications in image processing, see [27], [14], [12]. In this article we show that the solutions of all left-invariant linear evolution equations are given by convolution with the corresponding Green's function, which we explicitly derive. To cope with the cyclic boundary conditions in direction $\theta$, we follow two separate approaches. In the first approach, we expand the Green's kernel in series of Mathieu functions as described in the literature about the Mathieu equation [39]. The resulting series of Mathieu functions converges only slowly for group elements near the unity element, but we utilize this solution for our generalization (explained in Section 5) of a numerical algorithm of direction process by August [5] and derive a new and exact computation scheme ${ }^{1}$. In the second approach, we unwrap the torus $\mathbb{T}$ in $\theta$ and solve the partial differential equations for absorbing

\footnotetext{
${ }^{1}$ We provide the complete bi-orthogonal basis of eigenvectors of the matrix in this linear algorithm.
} 
$\theta$-boundaries at plus and minus infinity to eventually wrap the solution back onto the torus $\mathbb{T}$. Adding all rewrapped branches of the solution renders the Green's function for cyclic boundaries as a sum (which we explicitly calculate in subsection 4.2.1) of rapidly decaying terms. Both approaches are described for the special case of the direction process in Section 4. These approaches can be directly applied to the general case, including the stochastic processes for contour enhancement ([12]), where we derive the heat kernels on $\mathbb{R}^{2} \rtimes \mathbb{T}$.

In Section 4.3 we approximate the left-invariant basis of the Euclidean group generators by left-invariant generators of a Heisenberg-type group. The resulting equations render simple, analytic approximations of the exact Green's functions and provide explicit and simple formulas for the modes of so-called completion fields ([44]). A completion field is a probability density of line/contour propagation like the Green's function. The Green's function, however, is a probability density of a line with a given starting point and a given initial direction. A completion field is the probability density of collision of oriented grey-value particles moving from a source distribution, with oriented grey-value particles of a sink distribution. The modes of these completion fields represent the most probable connection between source and sink $([40])$. If both the source distribution and the sink distributions are delta-spikes in $\mathbb{R}^{2} \rtimes \mathbb{T}$, a completion field is the product of two (time-integrated) Green's functions.

The numeric algorithm that solves all linear, left-invariant, second order stochastic evolution equations on a discrete grid in Fourier space and its relation to our first analytic approach is the subject of Section 5 .

The reader who is not so much interested in the context of the underlying image analysis application can skip Section 2 .

2. Orientation scores. In many image analysis applications an object $U_{f} \in \mathbb{L}_{2}(G)$ defined on the 2D Euclidean motion group $G=\mathbb{R}^{2} \rtimes \mathbb{T}$ is constructed from a 2D greyvalue image $f \in \mathbb{L}_{2}\left(\mathbb{R}^{2}\right)$. Such an object provides an overview of all local orientations in an image. This is important for image analysis and perceptual organization ([35], [25], [38], [21], [18], [50], [42], [6]) and is inspired by our own visual system, in which receptive fields exist that are tuned to various locations and orientations ([46], [8]). In addition to the approach given in the introduction, other schemes to construct $U_{f}: \mathbb{R}^{2} \rtimes \mathbb{T} \rightarrow \mathbb{C}$ from an image $f: \mathbb{R}^{2} \rightarrow \mathbb{R}$ exist, but only a few methods put emphasis on the stability of the inverse transformation $U_{f} \mapsto f$.

In this section we provide an example on how to obtain such an object $U_{f}$ from an image $f$. This leads to the concept of invertible orientation scores, which we developed in previous work $([13],[18],[15])$ and which we briefly explain here.

An orientation score $U_{f}: \mathbb{R}^{2} \rtimes \mathbb{T} \rightarrow \mathbb{C}$ of an image $f: \mathbb{R}^{2} \rightarrow \mathbb{R}$ is obtained by means of an anisotropic convolution kernel $\check{\psi}: \mathbb{R}^{2} \rightarrow \mathbb{C}$ via

$$
U_{f}(g)=\int_{\mathbb{R}^{2}} \overline{\psi\left(R_{\theta}^{-1}(\mathbf{y}-\mathbf{x})\right)} f(\mathbf{y}) \mathrm{d} \mathbf{y}, \quad g=\left(\mathbf{x}, e^{i \theta}\right) \in G=\mathbb{R}^{2} \rtimes \mathbb{T}, R_{\theta} \in \mathrm{SO}(2),
$$


where $\check{\psi}(\mathbf{x})=\psi(-\mathbf{x})$. Assume $\psi \in \mathbb{L}_{1}\left(\mathbb{R}^{2}\right) \cap \mathbb{L}_{2}\left(\mathbb{R}^{2}\right)$. Then the transform $\mathcal{W}_{\psi}$ which maps image $f \in \mathbb{L}_{2}\left(\mathbb{R}^{2}\right)$ onto its orientation score $U_{f} \in \mathbb{L}_{2}\left(\mathbb{R}^{2} \rtimes \mathbb{T}\right)$ can be rewritten as

$$
U_{f}(g)=\left(\mathcal{W}_{\psi} f\right)(g)=\left(\mathcal{U}_{g} \psi, f\right)_{\mathbb{L}_{2}\left(\mathbb{R}^{2}\right)},
$$

where $g \mapsto \mathcal{U}_{g}$ is a unitary (group-)representation of the Euclidean motion group $\mathbb{R}^{2} \rtimes \mathbb{T}$ into $\mathbb{L}_{2}\left(\mathbb{R}^{2}\right)$ given by $\mathcal{U}_{g} f(\mathbf{y})=f\left(R_{\theta}^{-1}(\mathbf{y}-\mathbf{x})\right)$ for all $g=\left(\mathbf{x}, e^{i \theta}\right)$ and all $f \in \mathbb{L}_{2}\left(\mathbb{R}^{2}\right)$. Note that the representation $\mathcal{U}$ is reducible as it leaves the following closed subspaces invariant: $\left\{f \in \mathbb{L}_{2}\left(\mathbb{R}^{2}\right) \mid \operatorname{supp} \mathcal{F}[f] \subset B_{\mathbf{0}, \varrho}\right\}, \varrho>0$, where $B_{\mathbf{0}, \varrho}$ denotes the ball with center $\mathbf{0} \in \mathbb{R}^{2}$ and radius $\varrho>0$.

This differs from standard continuous wavelet theory, see for example [36] and [3], where the wavelet transform is constructed by means of a quasi-regular representation of the similitude group $\mathbb{R}^{d} \rtimes \mathbb{T} \times \mathbb{R}^{+}$, which is unitary, irreducible and square integrable (admitting the application of the more general results in [29]). For the image analysis this means that we do allow a stable reconstruction already at a single scale orientation score for a proper choice of $\psi$. In standard wavelet reconstruction schemes, however, it is not possible to obtain an image $f$ in a well-posed manner from a "fixed scale layer", that is, from $\mathcal{W}_{\psi} f(\cdot, \cdot, \sigma) \in \mathbb{L}_{2}\left(\mathbb{R}^{2} \rtimes \mathbb{T}\right)$, for fixed scale $\sigma>0 .^{2}$

Moreover, the general wavelet reconstruction results [29] do not apply to the transform $f \mapsto U_{f}$, since our representation $\mathcal{U}$ is reducible. In earlier work we therefore provided a general theory $([13],[10],[11])$ to construct wavelet transforms associated with admissible vectors/distributions. ${ }^{3}$ With these wavelet transforms we construct orientation scores $U_{f}: \mathbb{R}^{2} \rtimes \mathbb{T} \rightarrow \mathbb{C}$ by means of admissible line detecting vectors ${ }^{4} \psi \in \mathbb{L}_{2}\left(\mathbb{R}^{2}\right)$ such that the transform $\mathcal{W}_{\psi}$ is unitary onto the unique reproducing kernel Hilbert space $\mathbb{C}_{K}^{G}$ of functions on $G$ with reproducing kernel $K(g, h)=\left(\mathcal{U}_{g} \psi, \mathcal{U}_{h} \psi\right)$, which is a closed vector subspace of $\mathbb{L}_{2}(G)$. For the abstract construction of the unique reproducing kernel space $\mathbb{C}_{K}^{\mathbb{I}}$ on a set $\mathbb{I}$ (not necessarily a group) from a function of positive type $K: \mathbb{I} \times \mathbb{I} \rightarrow \mathbb{C}$, we refer to the early work of Aronszajn [4]. Here we only provide the essential Plancherel formula, which can also be found in a slightly different way in the work of Führ [28] for the wavelet transform $\mathcal{W}_{\psi}$ and which provides a more tangible description of the norm on $\mathbb{C}_{K}^{G}$ rather than the abstract one in [4]. To this end we note that we can write

$$
\left(\mathcal{W}_{\psi} f\right)\left(\mathbf{x}, e^{i \theta}\right)=\left(\mathcal{U}_{\left(\mathbf{x}, e^{i \theta}\right)} \psi, f\right)_{\mathbb{L}_{2}\left(\mathbb{R}^{2}\right)}=\left(\mathcal{F} \mathcal{T}_{x} \mathcal{R}_{\theta} \psi, \mathcal{F} f\right)_{\mathbb{L}_{2}\left(\mathbb{R}^{2}\right)}=\mathcal{F}^{-1}\left(\overline{\mathcal{R}_{\theta} \mathcal{F} \psi} \cdot \mathcal{F} f\right)(\mathbf{x})
$$

where the rotation and translation operators on $\mathbb{L}_{2}\left(\mathbb{R}^{2}\right)$ are defined by $\mathcal{R}_{\theta} f(\mathbf{y})=f\left(R_{\theta}^{-1} \mathbf{y}\right)$ and $\mathcal{T}_{\mathbf{x}} f(\mathbf{y})=f(\mathbf{y}-\mathbf{x})$. Consequently, we find that

$$
\begin{aligned}
\left\|\mathcal{W}_{\psi} f\right\|_{\mathbb{C}_{K}^{G}}^{2} & =\iint_{\mathbb{R}^{2}} \int_{\mathbb{T}}\left|\left(\mathcal{F} \mathcal{W}_{\psi} f\right)\left(\boldsymbol{\omega}, e^{i \theta}\right)\right|^{2} \mathrm{~d} \theta \frac{1}{M_{\psi}(\boldsymbol{\omega})} \mathrm{d} \boldsymbol{\omega} \\
& =\int_{\mathbb{R}^{2}} \int_{\mathbb{T}}|(\mathcal{F} f)(\boldsymbol{\omega})|^{2}\left|\mathcal{F} \psi\left(R_{\theta}^{T} \boldsymbol{\omega}\right)\right|^{2} \mathrm{~d} \theta \frac{1}{M_{\psi}(\boldsymbol{\omega})} \mathrm{d} \boldsymbol{\omega} \\
& =\int_{\mathbb{R}^{2}}|(\mathcal{F} f)(\boldsymbol{\omega})|^{2} \mathrm{~d} \boldsymbol{\omega}=\|f\|_{\mathbb{L}_{2}\left(\mathbb{R}^{2}\right)}^{2},
\end{aligned}
$$

\footnotetext{
${ }^{2}$ The same problem arises in linear scale space theory where it is impossible to reconstruct the original image in a stable $\mathbb{L}_{2}$-preserving matter from a fixed scale restriction $u_{f}^{\alpha}(\cdot, s)$ of a scale space representation $u_{f}^{\alpha}: \mathbb{R}^{d} \times \mathbb{R}^{+} \rightarrow \mathbb{R}$ obtained by an evolution equation on $\mathbb{R}^{d}$ generated by $-(-\Delta)^{\alpha}$, $0<\alpha \leq 1$ ([17]).

${ }^{3}$ This depends on whether images are assumed to be bandlimited or not; for full details see [11].

${ }^{4}$ Or rather admissible distributions $\psi \in \mathbb{H}^{-(1+\epsilon), 2}\left(\mathbb{R}^{2}\right), \epsilon>0$ if one does not want a restriction to bandlimited images.
} 
where $M_{\psi} \in C\left(\mathbb{R}^{2}, \mathbb{R}\right)$ is given by $M_{\psi}(\boldsymbol{\omega})=\int_{0}^{2 \pi}\left|\mathcal{F} \psi\left(R_{\theta}^{T} \boldsymbol{\omega}\right)\right|^{2} \mathrm{~d} \theta$. If $\psi$ is chosen such that $M_{\psi}=1$, then we gain $\mathbb{L}_{2}$-norm preservation. However, this is not possible as $\psi \in \mathbb{L}_{2}\left(\mathbb{R}^{2}\right) \cap \mathbb{L}_{1}\left(\mathbb{R}^{2}\right)$ implies that $M_{\psi}$ is a continuous function vanishing at infinity. Now, theoretically speaking, one can use a Gelfand-triple structure generated by $\sqrt{1+|\Delta|}$ to allow distributional wavelets $\psi \in \mathbb{H}^{-k}\left(\mathbb{R}^{2}\right), k>1$, with the property $M_{\psi}=1$, so that $\psi$ has equal length in each irreducible subspace (which uniquely correspond to the dual orbits of $S O(2)$ on $\mathbb{R}^{2}$ ); for details and generalizations see [11]. In practice, however, because of finite grid sampling, we can as well restrict $\mathcal{U}$ (which is well-defined) to the space of bandlimited images.

With this well-posed, unitary transformation between the space of images and the space of orientation scores at hand, we can perform image processing via orientation scores; see [16], [15], [16], [18], [35]. However, for the remainder of the article we assume that the object $U_{f}$ is some given function in $\mathbb{L}_{2}\left(\mathbb{R}^{2} \rtimes \mathbb{T}\right)$ and we write $U \in \mathbb{L}_{2}\left(\mathbb{R}^{2} \rtimes \mathbb{T}\right)$ rather than $U_{f} \in \mathbb{C}_{K}^{G}$. For all image analysis applications where an object $U_{f} \in \mathbb{L}_{2}\left(\mathbb{R}^{2} \rtimes\right.$ $\mathbb{T})$ is constructed from an image $f \in \mathbb{L}_{2}\left(\mathbb{R}^{2}\right)$, operators on the object $U \in \mathbb{L}_{2}\left(\mathbb{R}^{2} \rtimes \mathbb{T}\right)$ must be left-invariant to ensure Euclidean invariant image processing [13, p. 153]. This applies also to the cases where the original image cannot be reconstructed in a stable manner as in channel representations [24] and steerable tensor voting [26].

3. Left-invariant evolution equations on the Euclidean motion group. In order to construct the left-invariant evolution equations on the Euclidean motion group and to understand their structure, we first compute the left-invariant vector fields and their commutators on the Euclidean motion group. This structure has more or less been overlooked in previous work on the forward Kolmogorov equation of the well-known direction process ([40], [44], [51] and [5]). This structure will be relevant for our derivation of the solution of this evolution equation. Moreover, it provides a full overview on linear second order left-invariant evolution equations on the Euclidean motion group and thereby it provides more general and alternative left-invariant stochastic processes on the Euclidean motion group. We stress that all of these left-invariant stochastic processes are relevant for (image processing) applications. However, as explained in the introduction, here we focus on the ones relevant for contour-completion, such as the direction process.

Let $G=\mathbb{R}^{2} \rtimes \mathbb{T}$ be the Euclidean motion group with group product

$$
g g^{\prime}=\left(\mathbf{x}, e^{i \theta}\right)\left(\mathbf{x}^{\prime}, e^{i \theta^{\prime}}\right)=\left(\mathbf{x}+R_{\theta} \mathbf{x}^{\prime}, e^{i\left(\theta+\theta^{\prime}\right)}\right), g=\left(\mathbf{x}, e^{i \theta}\right), g^{\prime}=\left(\mathbf{x}^{\prime}, e^{i \theta^{\prime}}\right) \in \mathbb{R}^{2} \rtimes \mathbb{T},
$$

with unity element $e=(\mathbf{0}, 1)$ and $R_{\theta}=\left(\begin{array}{rr}\cos \theta & -\sin \theta \\ \sin \theta & \cos \theta\end{array}\right)$. Let $\left\{\mathbf{e}_{x}, \mathbf{e}_{y}\right\}$ be a positively oriented orthonormal basis in $\mathbb{R}^{2}$. Let $\mathbf{e}_{\theta}$ be a unit tangent vector at the unit element of $\mathbb{T}$. Then the tangent space at the unity element $T_{e}(G)$ is spanned by

$$
T_{e}(G)=\left\{A_{1}, A_{2}, A_{3}\right\}:=\left\{\mathbf{e}_{\theta}, \mathbf{e}_{x}, \mathbf{e}_{y}\right\} .
$$

As we will see, this basis yields the following left-invariant vector fields on $G:\left\{\mathbf{e}_{\theta}, \mathbf{e}_{\xi}, \mathbf{e}_{\eta}\right\}$, which are defined by

$$
\begin{aligned}
& \mathbf{e}_{\theta}\left(\mathbf{x}, e^{i \theta}\right)=\mathbf{e}_{\theta}, \\
& \mathbf{e}_{\xi}\left(\mathbf{x}, e^{i \theta}\right)=\cos \theta \mathbf{e}_{x}+\sin \theta \mathbf{e}_{y}, \\
& \mathbf{e}_{\eta}\left(\mathbf{x}, e^{i \theta}\right)=-\sin \theta \mathbf{e}_{x}+\cos \theta \mathbf{e}_{y},
\end{aligned}
$$


where we identified $T_{g=\left(\mathbf{x}, e^{i \theta}\right)}\left(\mathbb{R}^{2}, e^{i \theta}\right)$ with $T_{e}\left(\mathbb{R}^{2}, e^{i 0}\right)$ and $T_{g=\left(\mathbf{x}, e^{i \theta}\right)}(\mathbf{x}, \mathbb{T})$ with $T_{e}(\mathbf{0}, \mathbb{T})$, by means of parallel transport (on $\mathbb{R}^{2}$, respectively $\mathbb{T}$ ). The tangent space $T_{e}(G)$ is a $3 \mathrm{D}$ Lie algebra equipped with Lie product

$$
[A, B]=\lim _{t \downarrow 0} \frac{a(t) b(t)(a(t))^{-1}(b(t))^{-1}-e}{t^{2}},
$$

where $t \mapsto a(t)$, resp. $t \mapsto b(t)$, are any smooth curves in $G$ with $a(0)=b(0)=e$ and $a^{\prime}(0)=A$ and $b^{\prime}(0)=B$. The Lie products of the basis elements in (3.1) are

$$
\left[A_{1}, A_{2}\right]=A_{3}, \quad\left[A_{1}, A_{3}\right]=-A_{2}, \quad\left[A_{2}, A_{3}\right]=0 .
$$

The left, respectively right, regular representations of $G$ onto $\mathbb{L}_{2}(G)$ are given by $\mathcal{L}: G \rightarrow$ $\mathcal{B}\left(\mathbb{L}_{2}(G)\right): g \mapsto \mathcal{L}_{g}$ and $\mathcal{R}: G \rightarrow \mathcal{B}\left(\mathbb{L}_{2}(G)\right): g \mapsto \mathcal{R}_{g}$, where $\mathcal{L}_{g}$ and $\mathcal{R}_{g}$ are given by $\mathcal{L}_{g} \Phi(h)=\Phi\left(g^{-1} h\right)$ and $\mathcal{R}_{g} \Phi(h)=\Phi(h g)$, for all $g, h \in G$ and $\Phi \in \mathbb{L}_{2}(G)$. An operator $\Phi$ on $\mathbb{L}_{2}(G)$ is called left-invariant if it commutes with the left-regular representation, that is, $\Phi \circ \mathcal{L}_{g}=\mathcal{L}_{g} \circ \Phi$ for all $g \in G$. A vector field (now considered as differential operators) $\tilde{\mathcal{A}}$ on $G$ is called left-invariant if it satisfies

$$
\tilde{\mathcal{A}}_{g} \phi=\tilde{\mathcal{A}}_{e}\left(\phi \circ L_{g}\right)=\tilde{\mathcal{A}}_{e}(h \mapsto \phi(g h)),
$$

for all infinitely differentiable functions $\phi \in C_{c}^{\infty}\left(\Omega_{g}\right)$ where $\Omega_{g}$ is an open set around $g$ within $G$ and with the left multiplication $L_{g}: G \rightarrow G$ given by $L_{g}(h)=g h$.

Recall that the linear space of left-invariant vector fields $\mathcal{L}(G)$ equipped with the Lie product $[\tilde{A}, \tilde{B}]=\tilde{A} \tilde{B}-\tilde{B} \tilde{A}$ is isomorphic to $T_{e}(G)$ by means of the isomorphism

$$
T_{e}(G) \ni A \leftrightarrow \tilde{A} \in \mathcal{L}(G) \Leftrightarrow \tilde{A}_{g}(\phi)=A\left(\phi \circ L_{g}\right)=A(h \mapsto \phi(g h))
$$

for all smooth $\phi: G \supset \Omega_{g} \rightarrow \mathbb{R}$. By means of the derivative of the right regular representation $\mathrm{d} \mathcal{R}: T_{e}(G) \rightarrow \mathcal{L}(G)$ which is given by

$$
(\mathrm{d} \mathcal{R}(A) \Phi)(g)=\lim _{t \rightarrow 0} \frac{\left(\mathcal{R}_{\exp (t A)} \Phi\right)(g)-\Phi(g)}{t}, \quad A \in T_{e}(G), \Phi \in \mathbb{L}_{2}(G), g \in G,
$$

we obtain the corresponding basis of left-invariant vector fields on $G$ :

$$
\left\{\tilde{\mathcal{A}}_{1}, \tilde{\mathcal{A}}_{2}, \tilde{\mathcal{A}}_{3}\right\}:=\left\{\mathrm{d} \mathcal{R}\left(A_{1}\right), \mathrm{d} \mathcal{R}\left(A_{2}\right), \mathrm{d} \mathcal{R}\left(A_{3}\right)\right\}
$$

or explicitly in coordinates

$$
\left\{\tilde{\mathcal{A}}_{1}, \tilde{\mathcal{A}}_{2}, \tilde{\mathcal{A}}_{3}\right\}=\left\{\partial_{\theta}, \partial_{\xi}, \partial_{\eta}\right\}=\left\{\partial_{\theta}, \cos \theta \partial_{x}+\sin \theta \partial_{y},-\sin \theta \partial_{x}+\cos \theta \partial_{y}\right\}
$$

and indeed the Lie products of these basis elements are

$$
\left[\tilde{\mathcal{A}}_{1}, \tilde{\mathcal{A}}_{2}\right]=\tilde{\mathcal{A}}_{3}, \quad\left[\tilde{\mathcal{A}}_{1}, \tilde{\mathcal{A}}_{3}\right]=-\tilde{\mathcal{A}}_{2}, \quad\left[\tilde{\mathcal{A}}_{2}, \tilde{\mathcal{A}}_{3}\right]=0 .
$$

In this article we will derive exact analytic solutions and close analytic approximations which are much more tangible/easier to compute of the following second order linear left-invariant evolution equations

$$
\left\{\begin{array}{l}
\partial_{t} \wp=A \wp, \\
\lim _{t \downarrow 0} \wp(\cdot, t)=U(\cdot), \text { in } \mathbb{L}_{2}\left(\mathbb{R}^{2} \rtimes \mathbb{T}\right),
\end{array}\right.
$$


where the negative definite generator $A$ acting on $\mathbb{L}_{2}(G)$ is given by

$$
A=-\sum_{i=1}^{3} a_{i} \tilde{A}_{i}+\sum_{i, j=1}^{3} D_{i j} \tilde{A}_{i} \tilde{A}_{j}, \quad a_{i}, D_{i j} \in \mathbb{R}, i=1, \ldots, 3,
$$

where we restrict ${ }^{5}$ ourselves to the case $D_{i j}=D_{i i} \delta_{i j}$, with $D_{i i} \geq 0, i=1, \ldots, 3$, in which case the generator becomes

$$
A=\left[-a_{1} \partial_{\theta}-a_{2} \partial_{\xi}-a_{3} \partial_{\eta}+D_{11}\left(\partial_{\theta}\right)^{2}+D_{22}\left(\partial_{\xi}\right)^{2}+D_{33}\left(\partial_{\eta}\right)^{2}\right] .
$$

The first order part of the generator takes care of transport (convection) along the exponential curves, which are given by

$$
\begin{array}{r}
t \mapsto \exp \left(t\left(\sum_{i=1}^{3} a_{i} A_{i}\right)\right)=\left(x_{0}+\frac{a_{3}}{a_{1}}\left(\cos \left(a_{1} t+\theta_{0}\right)-\cos \theta_{0}\right)+\frac{a_{2}}{a_{1}}\left(\sin \left(a_{1} t+\theta_{0}\right)-\sin \theta_{0}\right),\right. \\
\left.y_{0}+\frac{a_{3}}{a_{1}}\left(\sin \left(a_{1} t+\theta_{0}\right)-\sin \theta_{0}\right)-\frac{a_{2}}{a_{1}}\left(\cos \left(a_{1} t+\theta_{0}\right)-\cos \theta_{0}\right), e^{i\left(a_{1} t+\theta_{0}\right)}\right),
\end{array}
$$

for $a_{1} \neq 0$, which is a circular spiral with radius $\frac{\sqrt{a_{2}^{2}+a_{3}^{2}}}{a_{1}}$ and central point

$$
\left(-\frac{a_{3}}{a_{1}} \cos \theta_{0}-\frac{a_{2}}{a_{1}} \sin \theta_{0}+x_{0}, \frac{a_{2}}{a_{1}} \cos \theta_{0}-\frac{a_{3}}{a_{1}} \sin \theta_{0}+y_{0}\right) .
$$

This result is easily deduced by the method of characteristics for first order PDE's. For $a_{1}=0$ we get a straight line in the plane $\theta=\theta_{0}$ :

$t \mapsto \exp \left(t\left(a_{2} A_{2}+a_{3} A_{3}\right)\right)=\left(x_{0}+t a_{2} \cos \theta_{0}-t a_{3} \sin \theta_{0}, y_{0}+t a_{2} \sin \theta_{0}+t a_{3} \cos \theta_{0}, e^{i \theta_{0}}\right)$, which coincides with (3.7) by taking the limit $a_{1} \rightarrow 0$.

The second order part takes care of diffusion in the Euclidean motion group $G$. Note that the non-commutative nature of the Euclidean motion group, recall (3.3), makes these evolution equations complicated. Furthermore we note that these evolution equations are indeed left-invariant as their generator is left-invariant (since it is constructed by linear combinations of products of left-invariant vector fields).

The motivation for considering these left-invariant evolution equations comes from probability theory. Consider the following stochastic equation on $\mathbb{R}^{2} \rtimes \mathbb{T}$ :

$$
\left\{\begin{array}{l}
\partial_{t} g(t)=\mathbf{a}(g(t), t)+B(g(t), t) \boldsymbol{\xi}(t), \\
g(0)=g_{0}
\end{array}\right.
$$

with $\boldsymbol{\xi}(t)=\left(\xi^{1}(t), \xi^{2}(t), \xi^{3}(t)\right)$, where the components are independently Gaussian white noise distributed, i.e. their expected value $\left\langle\xi^{i}(t)\right\rangle=0$ is zero and the correlation equals $\left\langle\xi^{i}\left(t_{0}\right) \xi^{j}\left(t_{1}\right)\right\rangle=2 \delta^{i j} D_{i i} \delta\left(t_{1}-t_{0}\right), D_{i i}>0, i, j=1, \ldots, 3$. The solution of (3.8) is

$$
g(t)=g_{0}+\int_{0}^{t} \mathbf{a}(g(s), s) \mathrm{d} s+\int_{0}^{t} B(g(s), s) \boldsymbol{\xi}(s) \mathrm{ds} .
$$

For the exact meaning of the stochastic differential equation (3.8) and the corresponding stochastic integral (3.9) and further details on stochastic processes, see [41]. In this article we shall only consider the case where $B(g(s), s)$ and $a(g(s), s)$ are left-invariant and not explicitly dependent on $s$. Then it does not matter whether one uses Stranovitch or Itŏ

\footnotetext{
${ }^{5}$ If one wants to include a priori curvature in the diffusion, one must consider non-diagonal $D$ ([27], $[12])$, but this is beyond the scope of this article.
} 


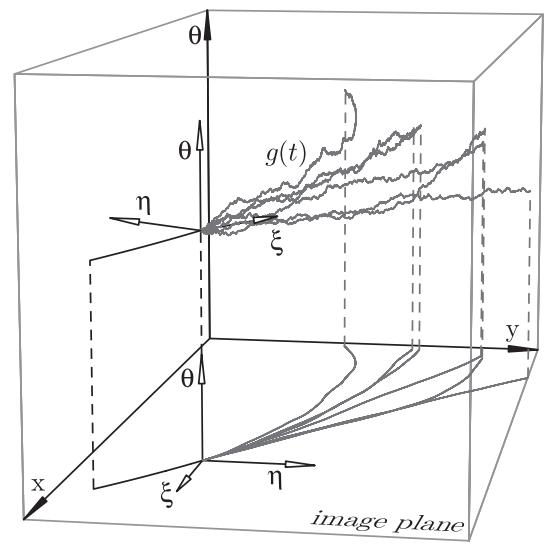

FIG. 2. Some random walks in $\mathbb{R}^{2} \rtimes \mathbb{T}$ (and their projection on $\mathbb{R}^{2}$ ) of direction processes with $\mathbf{a}=\left(\kappa_{0}, 1,0\right), D=\operatorname{diag}\left\{D_{11}, D_{22}, D_{33}\right\}$ (see (3.6), (3.10) and (3.11)) for various parameter settings of $\kappa_{0} \geq 0$ and $D_{i i}>0$.

calculus for the stochastic integrals, and Itŏ's formula for functions on the process $t \mapsto$ $(t, g(t))$ yields the following left-invariant evolution equation for the transition densities:

$$
\partial_{t} p\left(g, t \mid g_{0}, t_{0}\right)=-\sum_{i=1} \tilde{A}_{i} a_{i} p\left(g, t \mid g_{0}, t_{0}\right)+\frac{1}{2} \sum_{i, j=1}^{3} \tilde{A}_{i} \tilde{A}_{j}\left[B B^{T}\right]_{i j} p\left(g, t \mid g_{0}, t_{0}\right),
$$

which is known as the forward Kolmogorov equation of the stochastic process given by (3.8). In equation (3.10) we omitted the dependence of $a_{i}$ and $B_{i j}$ on $g$ because we have shown that to ensure left-invariance of the generator, the components of $a$ and $B$ with respect to the basis $\left\{\tilde{A}_{i}\right\}_{i=1}^{3}$ must be constant ([47]). The forward Kolmogorov equations of left-invariant stochastic processes (with constant $a, B, D=\frac{1}{2} B^{T} B$ ) are given by (3.4) and (3.5). These transition densities (3.10) are to be considered as limiting distributions of conditional probability densities of discrete processes (random walks) on the Euclidean motion group; see Figure 2. Consider for example the special case of the well-known direction process [40], [6], [50] where a random walker moves in the spatial plane along its current direction in the spatial plane (that is, along $\xi=x \cos \theta+y \sin \theta$ ) and where the average curvature of its path $^{6}$ is Gaussian distributed with variance $\sigma^{2}=2 D_{11}$ :

$$
\left\{\begin{array}{l}
e^{i \theta\left(s_{k}+\Delta s\right)}=e^{i\left(\theta\left(s_{k}\right)+\Delta s \eta\right)}, \quad \operatorname{Var}(\eta)=N \sigma^{2} \\
\mathbf{x}\left(s_{k}+\Delta s\right)=\mathbf{x}\left(s_{k}\right)+\Delta s\left(\begin{array}{c}
\cos \theta\left(s_{k}\right), \\
\sin \theta\left(s_{k}\right)
\end{array}\right) \\
\Delta s=\frac{L}{N}, \text { with } L \sim N E(\alpha), k=0, \ldots, N-1 .
\end{array}\right.
$$

The corresponding forward Kolmogorov equation is a special case (namely put $a_{1}=a_{3}=$ $\left.D_{22}=D_{33}=0\right)$ of (3.4) and (3.5).

In Section 4 we shall consider these evolution equations and provide the exact analytic solutions (and even more tangible analytic approximations) of both the evolution equations and their resolvent equations, which were strongly required (but not yet found)

\footnotetext{
${ }^{6}$ That is, $\bar{\kappa}=\frac{1}{L} \int_{0}^{L} k(s) \mathrm{ds}=\frac{1}{\mathrm{~L}} \int_{0}^{\mathrm{L}}|\dot{\theta}(\mathrm{s})| \mathrm{ds} \sim \mathcal{N}\left(0, \sigma^{2}\right)$, which explains the $N$ in (3.11).
} 
in the fields of applied mathematics and image analysis. In Subsection 4.6 we consider modifications of the direction process, where we include for example an a priori curvature $^{7} \kappa_{0} \geq 0$. For the case $a_{1}=a_{2}=a_{3}=0, D_{i i}>0, i=1,2,3$, so we are considering only diffusion, where $D_{i j}$ may even depend on $U$, we refer to [27], [12]. Furthermore in Section 5 we discuss an efficient method to compute the exact Green's functions in all cases (with periodic boundary conditions), where we explicitly put the connection with the exact solutions (with periodic boundary conditions) in the special cases above. We also point to Appendix A where we use Fourier transforms on the Euclidean motion group $\mathbb{R}^{2} \rtimes \mathbb{T}$ rather than Fourier transforms on $\mathbb{R}^{2}$ to obtain alternative (but similar) formulas for the solutions.

In Section 4 we shall use the following conventions:

- The unit-step function $\mathrm{u}: \mathbb{R} \rightarrow \mathbb{R}$ is given by $\mathrm{u}(x)=1$ if $x>0$ and $\mathrm{u}(x)=0$ if $x<0$ and $\mathrm{u}(0)=\frac{1}{2}$.

- The Fourier transform $\mathcal{F}: \mathbb{L}_{2}\left(\mathbb{R}^{2}\right) \rightarrow \mathbb{L}_{2}\left(\mathbb{R}^{2}\right)$ is almost everywhere defined by

$$
[\mathcal{F}(f)](\boldsymbol{\omega})=\hat{f}(\boldsymbol{\omega})=\frac{1}{(2 \pi)} \int_{\mathbb{R}^{2}} f(\mathbf{x}) e^{-i \boldsymbol{\omega} \cdot \mathbf{x}} \mathrm{d} \mathbf{x} .
$$

We use the following notation for Euclidean/polar coordinates in spatial and Fourier domains, respectively: $\mathbf{x}=(x, y)=(r \cos \phi, r \sin \phi), \boldsymbol{\omega}=\left(\omega_{x}, \omega_{y}\right)=$ $(\rho \cos \varphi, \rho \sin \varphi)$, with $\phi, \varphi \in[0,2 \pi), r, \rho>0$.

- Let $G=\mathbb{R}^{2} \rtimes \mathbb{T}$ be the Euclidean motion group. Then $\mathcal{D}(G)$ represents the vector space consisting of all infinitely differentiable functions with compact support within $G$. Let $a$ be a point on the manifold $G$. The Dirac distribution $\delta_{a}$ : $\mathcal{D}(G) \rightarrow \mathbb{C}$ is given by $\left\langle\delta_{a}, \phi\right\rangle=\delta_{a}(\phi)=\phi(a)$. Note that $\mathcal{D}(G)=\overline{\mathcal{D}\left(\mathbb{R}^{2}\right) \otimes \mathcal{D}(\mathbb{T})}$ and we write ${ }^{8}$

$$
\delta_{g^{\prime}}=\delta_{\left(x^{\prime}, y^{\prime}, e^{i \theta^{\prime}}\right)}=\delta_{x^{\prime}}^{x} \otimes \delta_{y^{\prime}}^{y} \otimes \delta_{\theta^{\prime}}^{\theta} .
$$

- The Gaussian kernel $G_{s}^{d}: \mathbb{R}^{d} \rightarrow \mathbb{R}^{+}$at scale $s=\frac{1}{2} \sigma^{2}$ is given by

$$
G_{s}^{d}(\mathbf{x})=\frac{1}{(4 \pi s)^{d / 2}} e^{-\frac{\|\mathbf{x}\|^{2}}{4 s}}
$$

4. The special case of the direction process. In this section we consider the evolution process $^{9}$

$$
\left\{\begin{array}{l}
\partial_{t} \wp=A \wp=\left(-\partial_{\xi}+D_{11}\left(\partial_{\theta}\right)^{2}\right) \wp, \\
\wp(\cdot, \cdot, 0, t)=\wp(\cdot, \cdot, 2 \pi, t) \text { for all } t>0, \\
\wp(\cdot, \cdot, \cdot, 0)=U(\cdot), \\
\wp(\cdot, t) \in \mathbb{L}_{2}(G), \text { for all } t>0 .
\end{array}\right.
$$

which is the forward Kolmogorov equation of the direction process, with probability density $\wp: G \times \mathbb{R}^{+} \rightarrow \mathbb{R}^{+}$, traveling time $T$ and initial condition $U \in \mathbb{L}_{2}(G)$. However if

\footnotetext{
${ }^{7}$ Relevant for our applications as it is possible to get curvature estimates from orientation scores ([27]).

${ }^{8}$ The upper indices in the Dirac distributions in the right hand side are indices to clarify the domain of the test functions on which these Dirac distributions apply.

${ }^{9}$ We use shorthand notation for partial derivatives $\partial_{\theta}=\frac{\partial}{\partial \theta}$.
} 
$T$ is negatively exponentially distributed ${ }^{10}, T \sim \mathrm{NE}(\alpha)$, i.e. the probability density of the random variable $T$ is given by $t \mapsto \alpha e^{-\alpha t}$, with expected traveling time $E(T)=\frac{1}{\alpha}$, then the unconditional probability density $p$ of finding an oriented particle with orientation $\theta$ and position $\mathbf{b}$ is given by

$$
\begin{aligned}
p(g) & =p(\mathbf{b}, \theta)=\int_{0}^{\infty} \wp(\mathbf{b}, \theta \mid T=t) p(T=t) \mathrm{dt} \\
& =\alpha \int_{0}^{\infty}\left[e^{t A} U\right](\mathbf{b}, \theta) e^{-t \alpha} \mathrm{dt}=-\alpha\left[(A-\alpha I)^{-1} U\right](\mathbf{b}, \theta) .
\end{aligned}
$$

So by application of the Laplace transform with respect to traveling time we obtain for the unconditional probability density $p$

$$
\left\{\begin{array}{l}
\left(\partial_{\xi}-D_{11}\left(\partial_{\theta}\right)^{2}+\alpha\right) p=\alpha U, \quad U \in \mathbb{L}_{2}(G), \\
p(\cdot, \cdot, 0)=p(\cdot, \cdot, 2 \pi), \\
p \in \mathbb{L}_{2}(G)
\end{array}\right.
$$

which is the resolvent equation of the strongly continuous (cf. Jørgensen [34, lemma 3.4] and the more detailed [20, IV.4.5]) semi-group on $\mathbb{L}_{2}(G)$ given by (4.1).

The problem (4.1) was first formulated (in the context of elastica in computer vision) by Mumford (cf. [40, p. 497]), who conjectured from further results in his paper that the solution may be expressed in elliptic functions of some kind, but he did not provide it. In image analysis Thornber and Williams [44] claimed to have found the analytic solution of this problem, but this claim is somewhat misleading: As they pointed out in a more careful exposition of their results [45, App. C], the Green's function for their random process on the space of positions and velocities is an approximation of the Green's function on the space of positions and directions. Next we show in detail that their kernels are Green's functions of left-invariant evolution equations on a group of Heisenberg-type rather than Green's functions of left-invariant evolution equations on the Euclidean motion group! Furthermore, we analyze the quality of these useful approximations for different parameter values and we provide generalizations and improvements (in Subsection 4.5).

Here we shall present the exact solution of both (4.2) and (4.1) in an explicit form by means of Fourier expansions (in the theta direction we use cosine elliptic functions, i.e. even Mathieu functions), which coincides with Mumford's conjecture [40, p. 497] on the existence of such a solution. At first glance these exact solutions may not seem very useful from the engineering point of view (but appearances are deceptive as their practical relevance become clear in Section 5). Therefore, in Subsection 4.2 we unwrap the torus yielding much more tangible solutions. Then we relate these solutions to the exact ones, yielding more tangible exact solutions. Moreover in Subsection 4.3 we will consider local Heisenberg-group approximations of the Euclidean motion group and solve for the Green's functions of the involved resolvent equations in spatial and Fourier domains, yielding somewhat more practical approximations of solutions for Green's functions of the resolvent equations and we use these solutions to derive the completion fields (as explained in the introduction) and their modes.

Both the generator $A$ and the operators $A-\alpha I$ and $A-\partial_{t}$ of the evolution system (4.1) are Hörmander operators of the second type. By the results of Hebisch [30] it now

\footnotetext{
${ }^{10}$ Which must be the case in a Markov process, as the only continuous memoryless distribution is the negatively exponential distribution.
} 
follows that the solution is a $G$-convolution in the distributional sense:

$$
\wp(\cdot, t)=\delta_{\exp \left(-t \partial_{\xi}\right)} * \tilde{K}_{t} * U,
$$

where the kernels $\tilde{K}_{t} \in \mathbb{L}_{2}(H)$ (for Gaussian estimates on $\tilde{K}_{t}$ and more details, see [30, Theorem 1.2, p. 3]), with $H$ the Lie group generated by the Lie algebra generated by $Y_{j, k}=\left\{\operatorname{ad}^{k}\left(\partial_{\xi}\right) \partial_{\theta}\right\}=\left\{\partial_{\theta}, \partial_{\eta}\right\}, k=0,1$, so $H=G$ and if we define $K_{t}=\delta_{\exp \left(-t \partial_{\xi}\right)} * \tilde{K}_{t}$, we get an ordinary ${ }^{11} G$-convolution with this kernel which is smooth on $G \backslash\{e\}$ due to the hypo-ellipticity of $A-\partial_{t}$. So we have

$$
\wp(g, t)=\left(K_{t} *_{G} U\right)(g),
$$

for all $g \in G$ and all $t>0$. Furthermore, the solution of (4.2) is a $G$-convolution with a Green's function $S_{\alpha, D_{11}}$ within $\mathbb{L}_{1}(G) \cap \mathbb{L}_{2}(G)$ which is (by a theorem of Hörmander; cf. [32]) smooth on $G \backslash\{e\}$ :

$$
\begin{aligned}
p(g) & =\left(S_{\alpha, D_{11}} *_{G} U\right)(g)=\int_{G} S_{\alpha, D_{11}}\left(h^{-1} g\right) U(h) \mathrm{d} \mu_{G}(h) \\
& =\frac{1}{2 \pi} \int_{\mathbb{R}^{2}} \int_{0}^{2 \pi} S_{\alpha, D_{11}}\left(R_{\theta^{\prime}}^{-1}\left(\mathbf{x}-\mathbf{x}^{\prime}\right), e^{i\left(\theta-\theta^{\prime}\right)}\right) U\left(\mathbf{x}^{\prime}, e^{i \theta^{\prime}}\right) \mathrm{d} \mathbf{x}^{\prime} \mathrm{d} \theta^{\prime}
\end{aligned}
$$

for all $g=\left(\mathbf{x}, e^{i \theta}\right) \in G$, where $\mu_{G}$ denotes the left-invariant Haar measure of the Euclidean motion group; for details see [13, p. 164]. This Green's function $g \mapsto S_{\alpha, D_{11}}(g)$, $g=\left(x, y, e^{i \theta}\right) \in \mathbb{R}^{2} \rtimes \mathbb{T}$, satisfies

$$
\left\{\begin{array}{l}
\left(\partial_{\xi}-D_{11}\left(\partial_{\theta}\right)^{2}+\alpha\right) S_{\alpha, D_{11}}=\alpha \delta_{e}, \\
S_{\alpha, D_{11}}(\cdot, \cdot, 0)=S_{\alpha, D_{11}}(\cdot, \cdot, 2 \pi), \\
S_{\alpha, D_{11}} \in \mathbb{L}_{2}(G) \cap \mathbb{L}_{1}(G) .
\end{array}\right.
$$

Notice that the Green's functions $K_{t}$ and $S_{\alpha, D_{11}}$ are connected via the Laplace transform: $S_{\alpha, D_{11}}=\alpha \mathcal{L}\left(t \mapsto K_{t}\right)(\alpha)$.

4.1. Explicit exact solution of the direction process. As the solution of (4.2) is given by a $G$-convolution with the Green's function $S_{\alpha, D_{11}}$, recall (4.4), it suffices to derive the unique solution of (4.5).

The first step is to perform a Fourier transform only with respect to the spatial part $\equiv \mathbb{R}^{2}$ of $G=\mathbb{R}^{2} \rtimes \mathbb{T}$, which yields $\hat{S}_{\alpha, D_{11}} \in \mathbb{L}_{2}(G) \cap C(G)$ given by

$$
\hat{S}_{\alpha, D_{11}}\left(\omega_{1}, \omega_{2}, \theta\right)=\mathcal{F}\left[S_{\alpha, D_{11}}(\cdot, \cdot, \theta)\right]\left(\omega_{1}, \omega_{2}\right) .
$$

Then $\hat{S}_{\alpha, D_{11}}$ satisfies

$$
\left\{\begin{array}{l}
\left(\cos \theta\left(i \omega_{x}\right)+\sin \theta\left(i \omega_{y}\right)-D_{11}\left(\partial_{\theta}\right)^{2}+\alpha\right) \hat{S}_{\alpha, D_{11}}\left(\omega_{x}, \omega_{y}, \cdot\right)=\frac{1}{2 \pi} \delta_{0}^{\theta}, \\
\hat{S}_{\alpha, D_{11}}\left(\omega_{x}, \omega_{y}, 0\right)=\hat{S}_{\alpha, D_{11}}\left(\omega_{x}, \omega_{y}, 2 \pi\right), \text { for all }\left(\omega_{x}, \omega_{y}\right) \in \mathbb{R}^{2}, \\
\hat{S}_{\alpha, D_{11}} \in \mathbb{L}_{2}(G) \cap C(G),
\end{array}\right.
$$

where we notice that $\mathcal{F}\left(\delta_{e}\right)=\frac{1}{2 \pi} 1_{\mathbb{R}^{2}} \otimes \delta_{0}$. Furthermore, we notice that the operator

$$
\mathcal{B}_{\omega_{x}, \omega_{y}}=\cos \theta\left(i \omega_{x}\right)+\sin \theta\left(i \omega_{y}\right)-D_{11}\left(\partial_{\theta}\right)^{2}+\alpha,
$$

${ }^{11}$ This is due to the fact that $\left\{\partial_{\theta}, \partial_{\eta}\right\}$ (are not commutative and) generate the full Lie algebra of $G$. Consider for example the case where $G=\mathbb{R}^{2}$ and $A=\left(\partial_{x}\right)^{2}+\partial_{y}$. Then $H=(\mathbb{R}, 0) \neq G$ and $(4.3)$ reads $\left(e^{t A} f\right)(x, y)=\int_{\mathbb{R}} G_{t}(x-v) f(v, y-t) \mathrm{dv}=\delta_{\exp \left(-t \partial_{y}\right)} * G_{t} * f(x, y)$, which is a singular convolution. Notice that in this case the Green's function of the resolvent is only singular at the origin as we have $-\lambda(A-\lambda I)^{-1} f=S_{\lambda} * f$, where $S_{\lambda}(x, y)=\lambda \mathcal{L}\left(t \mapsto\left(\delta_{\exp \left(-t \partial_{y}\right)} * G_{t}\right)(x, y)\right)(\lambda)=\lambda G_{y}(x) e^{-\lambda y} \mathrm{u}(y)$. 
for $\left(\omega_{x}, \omega_{y}\right) \in \mathbb{R}^{2}$ fixed is not a normal (so in particular not a self-adjoint) operator on $\mathbb{L}_{2}(\mathbb{T})$. However, it does satisfy

$$
\mathcal{B}_{\omega_{x}, \omega_{y}}^{*} \Theta=\overline{\mathcal{B}_{\omega_{x}, \omega_{y}} \bar{\Theta}} \text { for all } \Theta \in \mathbb{L}_{2}(\mathbb{T}) .
$$

The second step is to determine the complete basis of bi-orthogonal eigenfunctions within $\mathbb{L}_{2}(\mathbb{T})$ of operator $\mathcal{B}_{\omega_{x}, \omega_{y}}$ :

$$
\mathcal{B}_{\omega_{x}, \omega_{y}} \Theta=\lambda \Theta \Leftrightarrow\left(\cos \theta\left(i \omega_{x}\right)+\sin \theta\left(i \omega_{y}\right)-D_{11}\left(\partial_{\theta}\right)^{2}+\alpha\right) \Theta=\lambda \Theta,
$$

with $\Theta(0)=\Theta(2 \pi)$. Let $\varphi \in[0,2 \pi)$ be the polar angle in the Fourier domain, i.e. $\varphi=\arg \left(\omega_{x}+i \omega_{y}\right)$ and $\cos \varphi=\frac{\omega_{x}}{\|\boldsymbol{\omega}\|}, \sin \varphi=\frac{\omega_{y}}{\|\boldsymbol{\omega}\|}$. So then we have

$$
i\|\boldsymbol{\omega}\| \cos (\theta-\varphi)=i\left(\omega_{x} \cos \theta+\omega_{y} \sin \theta\right)
$$

and thereby (4.8) can be written

$$
\left\{\begin{array}{l}
\left(\partial_{\theta}^{2}-i \frac{\|\boldsymbol{\omega}\|}{D_{11}} \cos (\theta-\varphi)-\frac{\alpha}{D_{11}}\right) \Theta(\theta)=-\frac{\lambda}{D_{11}} \Theta(\theta) \\
\Theta(0)=\Theta(2 \pi)
\end{array}\right.
$$

Now set

$$
z=\frac{\theta-\varphi}{2} \in[0, \pi) \text { and } y(z)=y\left(\frac{\theta-\varphi}{2}\right)=\Theta(\theta)
$$

Then we have

$$
\left\{\begin{array}{l}
\left(\frac{1}{4} \partial_{z}^{2}-i \frac{\|\boldsymbol{\omega}\|}{D_{11}} \cos (2 z)-\frac{\alpha}{D_{11}}\right) y(z)=-\frac{\lambda}{D_{11}} y(z) \\
y(0)=y(\pi)
\end{array}\right.
$$

or equivalently

$$
\left\{\begin{array}{l}
y^{\prime \prime}(z)-2 h^{2} \cos (2 z) y(z)+a y(z)=0, \quad a=\frac{4(-\alpha+\lambda)}{D_{11}}, h^{2}=\frac{2\|\boldsymbol{\omega}\|}{D_{11}} i, \\
y(0)=y(\pi)
\end{array}\right.
$$

which is the well-known equation of Mathieu; cf. [39] and [1, Chapter 20]. A complete system of eigenfunctions consists of cosine elliptic functions ce $_{n}$ given by

$\mathrm{ce}_{n}\left(z ; h^{2}\right)=2^{\frac{1}{2}} \sum_{r=-\infty}^{\infty}\left(1+\delta_{r 0}\right)^{-1} c_{2 r}^{n}\left(h^{2}\right) \cos ((n+2 r) z)$, with $\lim _{r \rightarrow \infty}\left|c_{2 r}\right|^{\frac{1}{r}}=0, \quad n \in \mathbb{N} \cup\{0\}$,

where the Floquet exponent $\nu=n \in \mathbb{N} \cup\{0\}$; recall Floquet's Theorem ${ }^{12}$ [39, p. 101]. An alternative complete system of eigenfunctions are the Mathieu elliptic functions

$$
\mathrm{me}_{2 n}\left(z ; h^{2}\right)=\sum_{r=-\infty}^{\infty} c_{2 r}^{\nu=2 n}\left(h^{2}\right) e^{i(2 n+2 r) z}
$$

which satisfy $\operatorname{me}_{n}\left(z ; h^{2}\right)=2^{\frac{1}{2}} \operatorname{ce}_{n}\left(z ; h^{2}\right)$ for $n \in \mathbb{N} \cup\{0\}, \operatorname{me}_{-n}\left(z ; h^{2}\right)=i^{-1} 2^{\frac{1}{2}} \operatorname{se}_{n}\left(z ; h^{2}\right)$, where $\operatorname{se}_{2 n}\left(z ; h^{2}\right)$ denotes the sine-elliptic function (for details see [39]). By setting

$$
\begin{aligned}
& A_{0}^{2 n}\left(h^{2}\right)=2^{-\frac{1}{2}} c_{-2 n}^{2 n}\left(h^{2}\right), \\
& A_{r}^{m}\left(h^{2}\right)=2^{\frac{1}{2}} c_{r-m}^{m}\left(h^{2}\right) \text { for } r \neq 0, m=0,1,2, \ldots,
\end{aligned}
$$

\footnotetext{
${ }^{12}$ Due to the periodicity constraint the only allowed exponents are $\nu \in \mathbb{N} \cup\{0\}$.
} 
the Floquet solutions (4.11) can be rewritten as

$$
\operatorname{ce}_{2 n}\left(z ; h^{2}\right)=\sum_{r=0}^{\infty} A_{2 r}^{2 n}\left(h^{2}\right) \cos (2 r z), \quad \operatorname{ce}_{2 n+1}\left(z ; h^{2}\right)=\sum_{r=0}^{\infty} A_{2 r+1}^{2 n+1}\left(h^{2}\right) \cos ((2 r+1) z) .
$$

The coefficients $\left\{c_{2 r}^{2 n}\right\}$ and $\left\{A_{r}^{m}\right\}$ are determined by the 2-fold recursion systems: ${ }^{13}$

$$
\begin{aligned}
& \left\{\begin{array}{l}
\left(a_{2 n}\left(h^{2}\right)-4 r^{2}\right) c_{2 r}^{2 n}-h^{2}\left(c_{2 r+2}^{2 n}+c_{2 r-2}^{2 n}\right)=0, \quad r \in \mathbb{Z}, n \in \mathbb{Z}, \\
\lim _{r \rightarrow \pm \infty}\left|c_{2 r}^{2 n}\right|^{\frac{1}{r}}=0,
\end{array}\right. \\
& \left\{\begin{array}{l}
a_{2 n}\left(h^{2}\right) A_{0}^{2 n}-h^{2} A_{2}^{2 n}=0, \\
\left(a_{2 n}\left(h^{2}\right)-4\right) A_{2}^{2 n}-h^{2}\left(2 A_{0}^{2 n}+A_{4}^{2 n}\right)=0, \\
\left(a_{2 n}\left(h^{2}\right)-4 r^{2}\right) A_{2 r}^{2 n}-h^{2}\left(A_{2 r-2}^{2 n}+A_{2 r+2}^{2 n}\right)=0, \quad r \in \mathbb{N} \backslash\{1\}, n \in \mathbb{N} \cup\{0\},
\end{array}\right. \\
& \left\{\begin{array}{l}
\left(a_{2 n+1}\left(h^{2}\right)-1-h^{2}\right) A_{1}^{2 n+1}-h^{2} A_{3}^{2 n+1}=0, \\
\left(a_{2 n+1}\left(h^{2}\right)-(2 r+1)^{2}\right) A_{2 r+1}^{2 n+1}-h^{2}\left(A_{2 r-1}^{2 n+1}+A_{2 r+3}^{2 n+1}\right)=0, r \in \mathbb{N}, n \in \mathbb{N} \cup\{0\},
\end{array}\right.
\end{aligned}
$$

where the corresponding eigenvalues $a_{n}\left(h^{2}\right), n=0,1, \ldots$, are the countable solutions ${ }^{14}$ of the characteristic equations containing continued fractions:

$$
\begin{aligned}
& 0=-a+-2 h^{4} / /\left(2^{2}-a\right)+-h^{2} / /\left(4^{2}-a\right)+\ldots \text { for } \nu \text { even } \\
& 0=1+h^{2}-h^{4} / /\left(3^{2}-a\right)-h^{4} / /\left(5^{2}-a\right)-\ldots \text { for } \nu \text { odd } .
\end{aligned}
$$

Since these eigenvalues are analytical with respect to $h^{2}$ (with convergence radius $\rho_{n}$ ), they can be expanded in Taylor expansions in $h^{2}$ (the cases $n \neq 1$ even in $h^{4}$ ); see [39, pp. 120-121] or [1, p. 730]. Here we only give the expansions for $n \neq 1,2$ (for the cases $n=1,2$, see $[1$, p. 730$])$

$$
a_{n}\left(h^{2}\right)=n^{2}+\frac{1}{2\left(n^{2}-1\right)} h^{4}+\frac{5 n^{2}+7}{32\left(n^{2}-1\right)^{3}\left(n^{2}-4\right)} h^{8}+O\left(h^{12}\right) .
$$

The convergence radii $\rho_{n}$ (for example $\rho_{0} \approx 1.4688$ and $\rho_{2} \approx 3.7699$ ) are limited by the radii of the branching points of the analytic functions $\rho \mapsto a(\rho)$ and

$$
\liminf _{n \rightarrow \infty} \frac{\rho_{n}}{n^{2}} \geq 2.041823
$$

cf. [7] and [49]. The eigenfunctions $\left\{\mathrm{ce}_{n}\right\}_{n \in \mathbb{N} \cup\{0\}}$ and $\left\{\mathrm{me}_{2 n}\right\}_{n \in \mathbb{Z}}$ both form a complete bi-orthogonal system in $\mathbb{L}_{2}([0, \pi))$ :

$$
\begin{aligned}
& \left(\overline{\mathrm{ce}_{n}}, \mathrm{ce}_{m}\right)=\int_{0}^{\pi} \mathrm{ce}_{n}(z) \mathrm{ce}_{m}(z) \mathrm{dz}=\delta_{n m} \frac{\pi}{2}, \quad n, m=0,1,2,3, \ldots, \\
& \left(\overline{\mathrm{mĕ}_{2 n}}, \mathrm{me}_{2 m}\right)=\int_{0}^{\pi} \mathrm{me}_{2 n}(-z) \mathrm{me}_{2 m}(z) \mathrm{dz}=\delta_{n m} \pi, \quad n, m \in \mathbb{Z} .
\end{aligned}
$$

\footnotetext{
${ }^{13}$ These recursions follow directly by substitution of (4.11) in the Mathieu equation (4.10).

${ }^{14}$ The numeration in $n$ is rather a numeration over the eigenfunctions than a numeration over the Floquet exponents, as the only different relevant solutions are $\nu=1$ (the odd cases) and $\nu=0$ (the even cases).
} 
Moreover if a function $f$ is Lebesgue integrable on the interval $[0, \pi]$, we have for every $\nu>0$ and corresponding non-singular value of $h^{2}$ that

$$
\begin{aligned}
& f(z)=\sum_{n=0}^{\infty} \frac{1}{\pi} \int_{0}^{\pi} f(\tau) \mathrm{me}_{\nu+2 n}\left(-\tau ; h^{2}\right) \mathrm{d} \tau \mathrm{me}_{\nu+2 n}\left(z ; h^{2}\right), \\
& f(z)=\sum_{n=0}^{\infty} \frac{2}{\pi} \int_{0}^{\pi} f(\tau) \mathrm{ce}_{\nu+n}\left(\tau ; h^{2}\right) \mathrm{d} \tau \operatorname{ce}_{\nu+n}\left(z ; h^{2}\right)
\end{aligned}
$$

where for $h=0$ the resulting Fourier series, respectively Fourier cosine series,

$$
\begin{aligned}
& f(z)=\sum_{n=0}^{\infty} \frac{1}{\pi} \int_{0}^{\pi} f(\tau) e^{-i(\nu+2 n) \tau} \mathrm{d} \tau e^{i(\nu+2 n) t}, \\
& f(z)=\sum_{n=0}^{\infty} \frac{2}{\pi\left(1+\delta_{n 0}\right)} \int_{0}^{\pi} f(\tau) \cos ((\nu+n) \tau) \mathrm{d} \tau \cos ((\nu+n) \tau),
\end{aligned}
$$

are uniformly converging on $[0, \pi]$, from which it can be deduced that all convergence and summation properties (including the Gibss phenomenon) on standard Fourier series is carried over to the Mathieu series expansions; see [39, Satz 16, p. 128]. Note that the bi-orthogonality of the eigenfunctions follows from property (4.7):

$$
\left(\Theta_{n}, \overline{\Theta_{m}}\right)=\frac{1}{\overline{\lambda_{n}}}\left(\mathcal{B}_{\omega_{x}, \omega_{y}} \Theta_{n}, \overline{\Theta_{m}}\right)=\frac{1}{\overline{\lambda_{n}}}\left(\Theta_{n}, \overline{\mathcal{B}_{\omega_{x}, \omega_{y}} \Theta_{m}}\right)=\overline{\left(\frac{\lambda_{m}}{\lambda_{n}}\right)}\left(\Theta_{n}, \overline{\Theta_{m}}\right),
$$

so we have

$$
\text { either } 1-\frac{\lambda_{m}}{\lambda_{n}}=0 \text { or }\left(\Theta_{n}, \overline{\Theta_{m}}\right)=0,
$$

where we notice that operator $\mathcal{B}_{\omega_{x}, \omega_{y}}$ is coercive even for $\alpha=0$, so $\lambda_{n} \neq 0$ for all $n \in \mathbb{N}$. We stress that the bi-orthogonality only holds for eigenfunctions with different eigenvalues. For Mathieu equations with real-valued parameter $h$, it is well known that the corresponding real-valued eigenvalues are distinct. For the case of purely imaginary $h^{2}$, however, there exist countably many distinct singular values $\left(\rho_{n}\right) i=h_{2 n}^{2}, n=0,1,2, \ldots$, of purely imaginary $h^{2} \in \mathbb{R}^{+} i$ where the characteristic equation has double branching points where the two eigenvalues $a_{4 n}\left(h^{2}\right)$ and $a_{4 n+2}\left(h^{2}\right)$ merge, leading to two linearly independent eigenfunctions $\operatorname{ce}_{4 n}\left(\cdot ; h_{2 n}^{2}\right)$ and $\operatorname{ce}_{4 n+2}\left(\cdot ; h_{2 n}^{2}\right)$ with the same eigenvalue. According to (4.19) these eigenfunctions need not be bi-orthogonal to each other. Moreover, at these points (4.17) is no longer valid for $m=n$. The singular values $h_{2 n}^{2}=\left(\rho_{2 n}\right) i$ are the only branching points on the imaginary axis, and by (4.16) the series $\left\{h_{2 n}^{2}\right\}_{n \in \mathbb{N} \cup\{0\}}$ does not contain a density point. The other branching points where $a_{2 n+1}\left(h^{2}\right)$ and $a_{2 n+3}\left(h^{2}\right)$ coincide do not lie on the imaginary (or real) axis. For a complete overview via asymptotic analysis we refer to [33]; for precise numerical computation of the branching points we refer to [7]. Although the odd branching points do not lie on the imaginary axis, they do provide the convergence radii $\rho_{2 n+1}=\left|h_{2 n+1}^{2}\right|$ of the Taylor expansions in (4.15). If the purely imaginary $h^{2}$ passes a branching point $h_{n}^{2}$, the eigenvalues $a_{4 n}$ and $a_{4 n+2}$ become complex conjugate and in these cases one can use the following asymptotic formulae (for derivation see [33, pp. 117-119]):

$$
\overline{a_{2 m+2}(q)}=a_{2 m}(q) \sim 2 q+2(2 m+1) \sqrt{-q}-\frac{1}{4}\left(2 m^{2}+2 m+1\right)+O\left[(-q)^{\frac{-1}{2}}\right],
$$

with $q=h^{2}$ and $m=2 n$ and where the real part of $(-q)^{\frac{1}{2}}$ is positive, by placing a branch cut on the positive real axis, so $\sqrt{-t i}=e^{\frac{1}{2}\left(\log t-\frac{\pi}{2} i\right)}$ for $t>0$. For example, this 
asymptotic formula $(m=2)$ gives $a_{4}(20 i)=\overline{a_{6}}(20 i)=28.37+8.38 i$ whereas the exact eigenvalues are (to given precision) $28.96 \pm 8.35 i$, where we notice that the branching point where $a_{4}$ and $a_{6}$ coincide is given by $q=h^{2} \approx 17.3831 i$. Recall that the convergence radii grow with the order of $n^{2}$, so the asymptotic formula will become much more accurate for higher values of $n$.

So we conclude that a complete set of solutions of the eigenvalue problem (4.8) is given by

$$
\begin{cases}\Theta_{n}(\theta)=\operatorname{ce}_{n}\left(\frac{\theta-\varphi}{2}, h^{2}\right), & h^{2}=i \frac{2 \rho}{D_{11}}, \rho=\|\boldsymbol{\omega}\|, \\ -\lambda_{n}\left(h^{2}\right)=-\alpha-\frac{a_{n}\left(h^{2}\right) D_{11}}{4}<0, & n=0,1,2 \ldots,\end{cases}
$$

and that $\left\{\Theta_{n}\right\}$ form a complete bi-orthogonal basis on $\mathbb{L}_{2}([0,2 \pi])$ (or rather $\mathbb{L}_{2}(\mathbb{T})$ ) as long as $h^{2}$ is unequal to the branching points $h_{2 n}^{2}$.

Theorem 4.1. The Green's function $S_{\alpha, D_{11}} \in C^{\infty}(G \backslash\{e\})$ of the direction process, i.e. the unique solution of

$$
\left\{\begin{array}{l}
\left(\partial_{\xi}-D_{11}\left(\partial_{\theta}\right)^{2}+\alpha\right) S_{\alpha, D_{11}}=\alpha \delta_{e} \\
S_{\alpha, D_{11}}(\cdot, \cdot, 0)=S_{\alpha, D_{11}}(\cdot, \cdot, 2 \pi) \\
S_{\alpha, D_{11}} \in \mathbb{L}_{2}(G) \cap \mathbb{L}_{1}(G)
\end{array}\right.
$$

is given by

$$
S_{\alpha, D_{11}}(x, y, \theta)=\mathcal{F}^{-1}\left(\boldsymbol{\omega} \mapsto \frac{\alpha}{\pi^{2}} \sum_{n=0}^{\infty}\left(\frac{\operatorname{ce}_{n}\left(\frac{-\varphi}{2}, i \frac{2 \rho}{D_{11}}\right) \operatorname{ce}_{n}\left(\frac{\theta-\varphi}{2}, i \frac{2 \rho}{D_{11}}\right)}{\lambda_{n}}\right)\right)(x, y),
$$

with $\boldsymbol{\omega}=(\rho \cos \varphi, \rho \sin \varphi)$ and where the series converges in the $\mathbb{L}_{2}$-sense. The Green's function $S_{\alpha, D_{11}}$ is indeed a probability kernel as we have

$$
S_{\alpha, D_{11}} \geq 0 \text { and }\left\|S_{\alpha, D_{11}}\right\|_{\mathbb{L}_{1}(G)}=1 \quad \text { for all } \alpha>0 .
$$

Proof. Because $\left\{\Theta_{n}\right\}$ forms a complete bi-orthogonal system on $\mathbb{L}_{2}([0,2 \pi])$, we have

$$
2^{-1} \delta_{0}=\sum_{n=0}^{\infty} \frac{\Theta_{n}(0) \Theta_{n}(\cdot)}{\pi}
$$

in the distributional sense on $\mathcal{D}([0,2 \pi))$, i.e. we have

$$
2^{-1}\left\langle\delta_{0}, \phi\right\rangle=\lim _{N \rightarrow \infty}\left(\frac{1}{\pi} \sum_{n=0}^{N} \overline{\Theta_{n}(0) \Theta_{n}}(\cdot), \phi\right)=2^{-1} \phi(0),
$$

for all $\phi \in \mathcal{D}([0,2 \pi))$.

Now by the above derivations and (4.22) we have

$$
B_{\omega_{x}, \omega_{y}} \sum_{n=0}^{\infty} \frac{\Theta_{n}(0) \Theta_{n}(\cdot)}{\pi \lambda_{n}}=\sum_{n=0}^{\infty} \frac{\lambda_{n}}{\lambda_{n}} \frac{\Theta_{n}(0) \Theta_{n}(\cdot)}{\pi}=2^{-1} \delta_{0}=2^{-1} \delta_{0},
$$


for all $\omega_{x} \in \mathbb{R}$ and all $\omega_{y} \in \mathbb{R}$ and thereby we indeed get

$$
\begin{aligned}
\left\langle\left(\partial_{\xi}-D_{11}\left(\partial_{\theta}\right)^{2}+\alpha\right) S_{\alpha, D_{11}}, \phi\right\rangle & =\left\langle\mathcal{F}\left(\partial_{\xi}-D_{11}\left(\partial_{\theta}\right)^{2}+\alpha\right) S_{\alpha, D_{11}}, \mathcal{F}[\phi]\right\rangle \\
& =\left\langle\boldsymbol{\omega} \mapsto B_{\omega_{x}, \omega_{y}} \hat{S}_{\alpha, D_{11}}(\boldsymbol{\omega}, \cdot), \mathcal{F}\left[\phi_{1}\right] \otimes \phi_{2}\right\rangle \\
& =\left\langle\frac{1}{2 \pi} \otimes 2 \alpha \sum_{n=0}^{\infty} \frac{\lambda_{n}}{\lambda_{n}} \frac{\Theta_{n}(0) \Theta_{n}(\cdot)}{\pi}, \mathcal{F}\left[\phi_{1}\right] \otimes \phi_{2}\right\rangle \\
& =\phi_{2}(0) \frac{\alpha}{2 \pi} \int_{\mathbb{R}^{2}} \mathcal{F}\left[\phi_{1}\right](\boldsymbol{\omega}) \mathrm{d} \boldsymbol{\omega}=\alpha \phi_{2}(0) \phi_{1}(\mathbf{0})=\alpha \phi(e)
\end{aligned}
$$

for all test functions $\phi=\phi_{1} \otimes \phi_{2} \in \mathcal{D}\left(\mathbb{R}^{2}\right) \otimes \mathcal{D}(\mathbb{T})$. Now since $\overline{\mathcal{D}\left(\mathbb{R}^{2}\right) \otimes \mathcal{D}(\mathbb{T})}=\mathcal{D}\left(\mathbb{R}^{2} \rtimes \mathbb{T}\right)$, the result follows. Note that the series in (4.21) converges both in the $\mathbb{L}_{2}$-sense and uniformly on all compact sub-domains of $[0, \pi] \times \mathbb{R}^{2}$ that do not cross the lines

$$
\|\boldsymbol{\omega}\|=\frac{D_{11} h_{2 n}^{2}}{2 i}=\frac{D_{11} \rho_{2 n}}{2},
$$

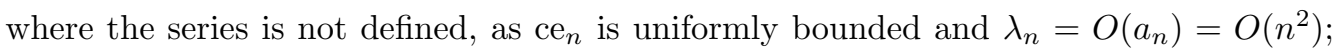
recall (4.15). Further, we note the rings (4.23) are a set of zero measure, so initially the solution $S_{\alpha, D_{11}}$ is almost everywhere given by (4.21), and since (Hörmander) it must be smooth on $G \backslash\{e\}$, it is everywhere given by (4.21).

Finally we notice that $\alpha>0$ and $-A+\alpha I>0$ imply that $-\alpha(A-\alpha I)^{-1}>0$ and thereby ${ }^{15} S_{\alpha, D_{11}}>0$; moreover a simple calculation yields

$$
\begin{aligned}
& \int_{G} S_{\alpha, D_{11}}(g) \mathrm{dg}=2 \pi \int_{0}^{2 \pi} \hat{S}_{\alpha, D_{11}}(0,0, \theta) \mathrm{d} \theta=\frac{\alpha}{2 \pi} \int_{0}^{2 \pi}\left(\sum_{n=0}^{\infty} \frac{e^{+\varphi n i} e^{(\theta-\varphi) n i}}{n^{2}+\alpha}\right) \mathrm{d} \theta \\
& =\frac{\alpha}{2 \pi} \sum_{n=0}^{\infty} \int_{0}^{2 \pi} \frac{e^{i n \theta}}{n^{2}+\alpha} \mathrm{d} \theta=\frac{\alpha}{\alpha}=1 .
\end{aligned}
$$

REMARK 4.2. Instead of using the bi-orthogonal basis (4.11), we may as well use the bi-orthogonal basis (4.12) in which case the solution can be written

$$
\hat{S}_{\alpha, D_{11}}(\boldsymbol{\omega}, \theta)=\frac{\alpha}{4 \pi^{2}} \sum_{n \in \mathbb{Z}} \frac{\mathrm{me}_{2 n}\left(\frac{-\varphi}{2}, \frac{2 i \rho}{D_{11}}\right) \mathrm{me}_{2 n}\left(\frac{-\theta+\varphi}{2}, \frac{2 i \rho}{D_{11}}\right)}{\alpha+\frac{a_{2 n}(q) D_{11}}{4}}, \quad q=\frac{2 i \rho}{D_{11}} .
$$

Analogously to the above we can construct the analytic solution of the time evolution process (4.1) which follows from Theorem 4.1 and the inverse Laplace transform.

TheOREM 4.3. The Green's function $\mathcal{S}_{D_{11}}=e^{t A} \delta_{e}$ of the direction process (4.1) is given by

$\mathcal{S}_{D_{11}}(x, y, \theta, t)=\mathcal{F}^{-1}\left(\boldsymbol{\omega} \mapsto \sum_{n=0}^{\infty} \frac{e^{\frac{-t a_{n}\left(h^{2}\right) D_{11}}{4}}}{2 \pi^{2}} \operatorname{ce}_{n}\left(\frac{-\varphi}{2}, \frac{2 \rho i}{D_{11}}\right) \operatorname{ce}_{n}\left(\frac{\theta-\varphi}{2}, \frac{2 \rho i}{D_{11}}\right)\right)(x, y)$,

with $\mathcal{S}_{D_{11}}>0$ and $\left\|\mathcal{S}_{D_{11}}(\cdot, \cdot, \cdot, t)\right\|_{\mathbb{L}_{1}(G)}=1$ for all $t>0$. This solution has the property that the solutions of the direction process (4.1) depend continuously on $D_{11} \geq 0$, i.e.

$$
\mathcal{S}_{D_{11}} \rightarrow \delta_{t}^{x} \otimes \delta_{0}^{y} \otimes \delta_{0}^{\theta}
$$

${ }^{15}$ These positive operators satisfy both $\forall_{U \in \mathbb{L}_{2}(G)}(A U, U)>0$ and $U>0 \Rightarrow A U>0$. 
in the distributional sense as $D_{11} \downarrow 0$.

Proof. The first part follows from Theorem 4.1 by means of the inverse Laplace transform. With respect to the second part we mention that if $D_{11}=0$, we obviously have $S_{\alpha, D_{11}=0}=\delta_{t}^{x} \otimes \delta_{0}$, i.e. the distributional Green's function of the direction process with $D_{11}$ is a deterministic unit speed transport of the $\delta$-distribution in $G$ along the direction $\xi$ which is along the $x$-axis at $\theta=0$.

Now we consider the case $D_{11}>0$ with $D_{11}$ tending to zero. Then by means of the asymptotic formula for $a_{n}(q)$ for $|q|$ large $\left[1\right.$, p. 726], we have $a_{n}(q) \sim-2 q$, so

$$
e^{-t \frac{a_{n}\left(h^{2}\right) D_{11}}{4}} \rightarrow e^{i \rho t}
$$

where we recall that $h^{2}=\frac{2 i \rho}{D_{11}}$, and by (4.17) we have that

$$
\sum_{n=0}^{\infty} \operatorname{ce}_{n}\left(-\frac{\varphi}{2}, \frac{2 \rho i}{D_{11}}\right) \operatorname{ce}_{n}\left(\frac{\theta-\varphi}{2}, \frac{2 \rho i}{D_{11}}\right)=\frac{\pi}{2} \delta_{0}^{\theta}
$$

for all $\rho, D_{11}>0$, in the distributional sense on $\mathcal{D}([0,2 \pi))$. Now $\lim _{D_{11} \downarrow 0} \hat{S}_{D_{11}}\left(\omega_{x}, \omega_{y}, \theta\right)$ is independent of $\omega_{y}$ and therefore it follows by the asymptotic behavior (4.25) that

$$
\mathcal{S}_{D_{11}} \rightarrow \mathcal{F}^{-1}\left[e^{-i \omega_{x} t} \otimes \frac{1}{2 \pi} \otimes \delta_{0}^{\theta}\right]=\delta_{t}^{x} \otimes \delta_{0}^{y} \otimes \delta_{0}^{\theta}
$$

in the distributional sense on $\mathcal{D}\left(\mathbb{R}^{2} \rtimes \mathbb{T}\right)$ as $D_{11} \downarrow 0$.

4.2. Unwrapping the torus. From the computational point of view there exist several disadvantages of the exact solutions in Theorem 4.1 and Theorem 4.3. First it requires a lot of samplings from various periodic Mathieu functions with imaginary parameters and the standard expansions of the coefficients in $h^{2}=i \frac{2 \rho}{D_{11}}$ are only valid before the first branching point. Secondly, the bi-orthogonal series expansion slowly converges close to the unity element where the Green's function has a singularity.

To overcome these computational deficiencies, we first assume $\theta \in \mathbb{R}$ rather than $\theta \in[0,2 \pi)$ and replace the periodic boundary conditions in (4.2) by the condition

$$
p(\cdot, \theta) \rightarrow 0 \text { unformly on compact domains within } \mathbb{R}^{2} \text { as }|\theta| \rightarrow \infty,
$$

and secondly we can expand the exact Green's function $S_{\alpha, D_{11}}$ as an infinite sum over $2 \pi$-shifts of the solution $S_{\alpha, D_{11}}^{\infty}$ for the unbounded case:

$$
S_{\alpha, D_{11}}\left(x, y, e^{i \theta}\right)=\lim _{N \rightarrow \infty} \sum_{k=-N}^{N} S_{\alpha, D_{11}}^{\infty}(x, y, \theta-2 k \pi) .
$$

Typically, $D_{11} / \alpha$ is small and this sum may be truncated at $k=0$ : For $D_{11} / \alpha$ small the homotopy number of the path of an orientation of the random walker is most likely to be 0 . However, theoretically, the further $D_{11} / \alpha>0$ increases, the larger the probability that the orientation of the random walker makes one or more rounds on the torus, that is, the more terms are required in the series expansion in (4.27). For parameter ranges relevant for image analysis applications, the series can already be truncated at $N=0,1$ or at most at $N=2$ for almost exact approximation. But, we will also provide a simple expression for the true exact solution, that is, without truncating the series (4.27) in Subsection 4.2.1. 
The following lemma will be used to construct the unique solution $S_{D_{11}, \alpha}^{\infty}: \mathbb{R}^{2} \times \mathbb{R} \rightarrow$ $\mathbb{R}^{+}$which satisfies (4.26).

Lemma 4.4. Let $\beta>0$ and let $a, c \in \mathbb{R}$. Then the unique Green's function $\mathcal{G} \in C^{\infty}(\mathbb{R} \backslash$ $\{0,0,0\}, \mathbb{C})$ that satisfies

$$
\left\{\begin{array}{l}
\left(-i a \sin \theta+\left(\partial_{\theta}\right)^{2}-i c \cos \theta-\beta\right) \mathcal{G}=\delta_{0} \\
\mathcal{G}(\theta) \rightarrow 0 \text { as }|\theta| \rightarrow \infty
\end{array}\right.
$$

is given by

$$
\begin{aligned}
\mathcal{G}(\theta) & =\frac{1}{i W_{-4 \beta, 2 i R}}\left[\mathrm{me}_{\nu}\left(\frac{\gamma}{2}, 2 i R\right) \mathrm{me}_{-\nu}\left(\frac{\gamma-\theta}{2}, 2 i R\right) \mathrm{u}(\theta)\right. \\
& \left.+\mathrm{me}_{-\nu}\left(\frac{\gamma}{2}, 2 i R\right) \mathrm{me}_{\nu}\left(\frac{\gamma-\theta}{2}, 2 i R\right) \mathrm{u}(-\theta)\right]
\end{aligned}
$$

with $R=\sqrt{a^{2}+c^{2}}>0$, with $\gamma=\arg (c+i a)$ and where the non-periodic complex-valued Mathieu functions are by definition ${ }^{16}$ ([39, p. 115], [1, p. 732]) given by

$$
\mathrm{me}_{ \pm \nu}(z, 2 i R)=\mathrm{ce}_{\nu}(z, 2 i R) \pm i \mathrm{se}_{\nu}(z, 2 i R)=\sum_{r=-\infty}^{\infty} c_{2 r}^{ \pm \nu}(2 i R) e^{i( \pm \nu+2 r) z}
$$

with $\nu$ the Floquet exponent ${ }^{17}$ of the Mathieu equation ${ }^{18} \nu=\nu(-4 \beta, 2 i R)$ and where $W_{-4 \beta, 2 i R}$ equals the Wronskian of $z \mapsto \mathrm{ce}_{\nu}(z, 2 i R)$ and $z \mapsto \mathrm{se}_{\nu}(z, 2 i R)$, so

$$
W_{-4 \beta, 2 i R}=\mathrm{ce}_{\nu(-4 \beta, 2 i R)}(0,2 i R) \operatorname{se}_{\nu(-4 \beta, 2 i R)}^{\prime}(0,2 i R) .
$$

Proof. The system (4.28) is equivalent to

$$
\left\{\begin{array}{l}
\left(\left(\partial_{\theta}\right)^{2}-i R \cos (\theta-\gamma)-\beta\right) \mathcal{G}=\delta_{0} \\
\mathcal{G}(\theta) \rightarrow 0 \text { as }|\theta| \rightarrow \infty
\end{array}\right.
$$

The linear space of infinitely differentiable solutions of

$$
\mathcal{G}^{\prime \prime}(\theta)-(i R \cos (\theta-\gamma)+\beta) \mathcal{G}(\theta)=0
$$

is spanned by $\left\{\theta \mapsto \operatorname{me}_{\nu}\left(\frac{\gamma-\theta}{2}, 2 i R\right), \theta \mapsto \mathrm{me}_{-\nu}\left(\frac{\gamma-\theta}{2}, 2 i R\right)\right\}$. Now we notice that $\nu=$ $\nu(-4 \beta, 2 i R)$, for $\beta>0, R>0$, lies on the positive imaginary axis, and as a result the only solutions that vanish as $\theta \rightarrow+\infty$ are given by $\theta \mapsto \mathrm{me}_{\nu}\left(\frac{\gamma-\theta}{2}, 2 i R\right)$, whereas the only solutions that vanish as $\theta \rightarrow-\infty$ are given by $\theta \mapsto \operatorname{me}_{-\nu}\left(\frac{\gamma-\theta}{2}, 2 i R\right)$. Furthermore by the Hörmander theorem it follows that $\mathcal{G}$ must be infinitely differentiable outside the origin, so we must have

$$
G(\theta)=C_{1} \mathrm{me}_{\nu}\left(\frac{\gamma-\theta}{2}, 2 i R\right) \mathrm{u}(-\theta)+\mathrm{C}_{2} \mathrm{me}_{-\nu}\left(\frac{\gamma-\theta}{2}, 2 \mathrm{iR}\right) \mathrm{u}(\theta),
$$

\footnotetext{
${ }^{16}$ There exist several definitions of Mathieu solutions (for an overview see [1, p. 744, Table 20.10]), each with different normalizations. In this article we always follow the consistent conventions by Meixner and Schaefke [39]. However, for example, Mathematica 5.2 chooses an unspecified convention not consistent with bi-orthogonality. This requires a slight modification of (4.30); see [2].

${ }^{17}$ Since the Floquet exponents come in conjugate pairs and since the Mathieu exponent $\nu(a, q)$ is purely imaginary for purely imaginary $q$ (for a proof see [14, Appendix A, Lemma A.3]), we may assume that the Floquet exponent for imaginary parameter $q$ lies on the positive imaginary axis. This convention is used throughout this article. For details on how to compute the Floquet exponent $\nu(a, q)$ of Mathieu's equation, see [1, pp. 727-728].

${ }^{18}$ Since we dropped the periodicity constraint, we no longer have $\nu \in \mathbb{N}$.
} 
where we recall that $\mathrm{u}$ is the unit step function (or Heaviside's distribution). By applying the Fourier transform with respect to $\theta$, it directly follows that $\hat{G} \in \mathbb{L}_{1}(\mathbb{R})$ and thereby it follows that $\mathcal{G}$ must be a continuous function vanishing at infinity. Now $\mathcal{G}$ is continuous at $\theta=0$ iff $C_{1}=\lambda \mathrm{me}_{-\nu}\left(\frac{\gamma}{2}, 2 i R\right), C_{2}=\lambda \mathrm{me}_{\nu}\left(\frac{\gamma}{2}, 2 i R\right)$ for some $\lambda \neq 0$, to be determined. The constant $\lambda$ directly follows by (4.32) and (4.28):

$$
\begin{aligned}
\delta_{0}= & \left(-i a \sin \theta+\left(\partial_{\theta}\right)^{2}-i c \cos \theta-\beta\right) \mathcal{G} \\
= & -\frac{\lambda}{2}\left(\mathrm{me}_{\nu}\left(\frac{\gamma}{2}, 2 i R\right) \mathrm{me}_{-\nu}^{\prime}\left(\frac{\gamma}{2}, 2 i R\right)-\mathrm{me}_{-\nu}\left(\frac{\gamma}{2}, 2 i R\right) \mathrm{me}_{\nu}^{\prime}\left(\frac{\gamma}{2}, 2 i R\right)\right) \delta_{0} \\
& +0 \cdot \mathrm{u}(-\theta)+0 \cdot \mathrm{u}(\theta)+\lambda \mathcal{G}(\theta)\left(\delta_{0}^{\prime}-\delta_{0}^{\prime}\right) \\
= & -(\lambda / 2) W\left[\mathrm{me}_{\nu}(\cdot, 2 i R), \mathrm{me}_{-\nu}(\cdot, 2 i R)\right] \delta_{0} \\
= & i \lambda W\left[\mathrm{ce}_{\nu}(\cdot, 2 i R), \mathrm{se}_{\nu}(\cdot, 2 i R)\right] \delta_{0}=i \lambda W_{-4 \beta, 2 i R} \delta_{0},
\end{aligned}
$$

where the Wronskian is given by $W[p, q]=p q^{\prime}-q p^{\prime}$ from which the result follows. Finally, the Wronskian is independent of $z$ and (4.31) follows at $z=0$.

Now by setting $\gamma=\varphi, R=\frac{\rho}{D_{11}}$ and $\beta=\frac{\alpha}{D_{11}}$ (recall that $\rho$ and $\varphi$ are the polar coordinates in the Fourier domain, i.e. $\boldsymbol{\omega}=(\rho \cos \varphi, \rho \sin \varphi))$, we get

Theorem 4.5. The solution $S_{\alpha, D_{11}}^{\infty}: \mathbb{R}^{3} \backslash\{0,0,0\}$ of the problem

$$
\left\{\begin{array}{l}
\left(\partial_{\xi}-D_{11}\left(\partial_{\theta}\right)^{2}+\alpha\right) S_{\alpha, D_{11}}^{\infty}=\alpha \delta_{e}, \\
S_{\alpha, D_{11}}^{\infty}(\cdot, \cdot, \theta) \rightarrow 0 \text { uniformly on compacta as }|\theta| \rightarrow \infty \\
S_{\alpha, D_{11}}^{\infty} \in \mathbb{L}_{1}\left(\mathbb{R}^{3}\right)
\end{array}\right.
$$

is given by

$$
S_{\alpha, D_{11}}^{\infty}(x, y, \theta)=\mathcal{F}^{-1}\left[\left(\omega_{x}, \omega_{y}\right) \mapsto \hat{S}_{\alpha, D_{11}}^{\infty}\left(\omega_{x}, \omega_{y}, \theta\right)\right](x, y)
$$

where

$$
\begin{aligned}
\hat{S}_{\alpha, D_{11}}^{\infty}\left(\omega_{x}, \omega_{y}, \theta\right)=\frac{-\alpha}{2 \pi D_{11}} & \frac{1}{i W_{\frac{-4 \alpha}{D_{11}}, \frac{2 i \rho}{D_{11}}}}\left[\mathrm{me}_{\nu}\left(\frac{\varphi}{2}, i \frac{2 \rho}{D_{11}}\right) \mathrm{me}_{-\nu}\left(\frac{\varphi-\theta}{2}, i \frac{2 \rho}{D_{11}}\right) \mathrm{u}(\theta)\right. \\
& \left.+\mathrm{me}_{-\nu}\left(\frac{\varphi}{2}, i \frac{2 \rho}{D_{11}}\right) \mathrm{me}_{\nu}\left(\frac{\varphi-\theta}{2}, i \frac{2 \rho}{D_{11}}\right) \mathrm{u}(-\theta)\right]
\end{aligned}
$$

with Floquet exponent $\nu=\nu\left(\frac{-4 \alpha}{D_{11}}, \frac{2 i \rho}{D_{11}}\right)$ and Wronskian $W_{\frac{-4 \alpha}{D_{11}}, \frac{2 i \rho}{D_{11}}}$; recall (4.31).

Notice that $\hat{S}_{\alpha, D_{11}}^{\infty}$ has a much simpler form than the Fourier transform of the true Green's function with periodic boundary conditions (4.21) and clearly (4.27) together with Theorem 4.5 is preferable over Theorem 4.1 as the series converges much faster and now we no longer have numerical problems nearby the branching points on the imaginary axis, that is, on the circles $\rho=\|\boldsymbol{\omega}\|=\frac{D_{11} \varrho_{2 n}}{2}$ (recall (4.23)). However, if one is not interested in a homotopy decomposition of the exact Green's function $S_{\alpha, D_{11}}$, the next section provides a much better formula for $S_{\alpha, D_{11}}$.

4.2.1. The exact solution for the resolvent Green's function $S_{\alpha, D_{11}}$ of the direction process. The summation (4.27) can be calculated by means of the property me $\operatorname{ta}_{ \pm \nu}\left(\frac{\varphi-\theta}{2}+k \pi\right)$ $=e^{ \pm i \nu k} \mathrm{me}_{\nu}\left(\frac{\varphi-\theta}{2}\right)$ due to the well-known Floquet theorem! Now by our convention on Mathieu functions (similar to [39]) we have (4.30) and $\nu\left(\frac{-4 \alpha}{D_{11}}, \frac{2 i \rho}{D_{11}}\right)$ lies on the positive imaginary axis in the complex plane, so $\left|e^{i \nu}\right|<1$ and $\sum_{k=0}^{\infty} r^{k}=\frac{1}{1-r}$ for $r=e^{i \nu}$. This directly yields the following practical solution $S_{\alpha, D_{11}}\left(\mathbf{x}, e^{i \theta}\right)=\mathcal{F}^{-1}\left[\boldsymbol{\omega} \mapsto \hat{S}_{\alpha, D_{11}}\left(\boldsymbol{\omega}, e^{i \theta}\right)\right](\mathbf{x})$ 
with

$$
\begin{aligned}
& \hat{S}_{\alpha, D_{11}, e^{i \theta}}\left(\boldsymbol{\omega}, e^{i \theta}\right)=\frac{\alpha}{2 \pi D_{11} \operatorname{ce}_{\nu}\left(0, \frac{2 \rho i}{D_{11}}\right) \operatorname{se}_{\nu}^{\prime}\left(0, \frac{2 \rho i}{D_{11}}\right)} \\
& \times\left\{\left(-\cot \left(\frac{\nu \pi}{2}\right)\left(\operatorname{ce}_{\nu}\left(\frac{\varphi}{2}, \frac{2 \rho i}{D_{11}}\right) \operatorname{ce}_{\nu}\left(\frac{\varphi-\theta}{2}, \frac{2 \rho i}{D_{11}}\right)+\operatorname{se}_{\nu}\left(\frac{\varphi}{2}, \frac{2 \rho i}{D_{11}}\right) \operatorname{se}_{\nu}\left(\frac{\varphi-\theta}{2}, \frac{2 \rho i}{D_{11}}\right)\right)\right.\right. \\
& \left.\quad+\operatorname{ce}_{\nu}\left(\frac{\varphi}{2}, \frac{2 \rho i}{D_{11}}\right) \operatorname{se}_{\nu}\left(\frac{\varphi-\theta}{2}, \frac{2 \rho i}{D_{11}}\right)-\operatorname{se}_{\nu}\left(\frac{\varphi}{2}, \frac{2 \rho i}{D_{11}}\right) \operatorname{ce}_{\nu}\left(\frac{\varphi-\theta}{2}, \frac{2 \rho i}{D_{11}}\right)\right) \mathrm{u}(\theta) \\
& +\left(-\cot \left(\frac{\nu \pi}{2}\right)\left(\operatorname{ce}_{\nu}\left(\frac{\varphi}{2}, \frac{2 \rho i}{D_{11}}\right) \operatorname{ce}_{\nu}\left(\frac{\varphi-\theta}{2}, \frac{2 \rho i}{D_{11}}\right)-\operatorname{se}_{\nu}\left(\frac{\varphi}{2}, \frac{2 \rho i}{D_{11}}\right) \operatorname{se}_{\nu}\left(\frac{\varphi-\theta}{2}, \frac{2 \rho i}{D_{11}}\right)\right)\right. \\
& \left.\left.\quad+\operatorname{ce}_{\nu}\left(\frac{\varphi}{2}, \frac{2 \rho i}{D_{11}}\right) \operatorname{se}_{\nu}\left(\frac{\varphi-\theta}{2}, \frac{2 \rho i}{D_{11}}\right)+\operatorname{se}_{\nu}\left(\frac{\varphi}{2}, \frac{2 \rho i}{D_{11}}\right) \operatorname{ce}_{\nu}\left(\frac{\varphi-\theta}{2}, \frac{2 \rho i}{D_{11}}\right)\right) \mathrm{u}(-\theta)\right\} .
\end{aligned}
$$

For details and similar solutions including a priori curvature $\kappa_{0}>0$ (as we consider in Section 4.6) following the Mathieu conventions of Mathematica 5.2, see [2].

4.2.2. Singularities of the Green's functions at the unity element. The function $\hat{S}_{\alpha, D_{11}}^{\infty}(\cdot, \theta)$ vanishes at $\|\boldsymbol{\omega}\| \rightarrow \infty$ for all $\theta \in \mathbb{R}$, but rather slowly, and $S_{\alpha, D_{11}}^{\infty}$ has a singularity at its origin (the unity element $e$ ). This singularity has disadvantages in applications and can be avoided by applying some extra spatial isotropic diffusion $s>0$ :

$$
\begin{aligned}
e^{s \partial_{\xi}^{2}+s \partial_{\eta}^{2}} S_{\alpha, D_{11}}^{\infty}(x, y, \theta) & =\left(e^{s \Delta} S_{\alpha, D_{11}}^{\infty}\right)(x, y, \theta) \\
& =\mathcal{F}^{-1}\left[\left(\omega_{x}, \omega_{y}\right) \mapsto e^{-s \rho^{2}} \hat{S}_{\alpha, D_{11}}^{\infty}\left(\omega_{x}, \omega_{y}, \theta\right)\right](x, y),
\end{aligned}
$$

with diffusion constant $s>0$. In general we notice that the left-invariant operators

$$
U \mapsto-\alpha e^{s B}(A-\alpha I)^{-1} U
$$

or $U \mapsto-\alpha e^{s B} e^{t A} U$ with $A=\sum_{i=1}^{3}-a_{i} A_{i}+D_{i i}\left(A_{i}\right)^{2}$ and $B=\sum_{i=1}^{3} \tilde{D}_{i i}\left(A_{i}\right)^{2}$, with $s \tilde{D}_{i i}$ relatively small, are more suitable for image analysis purposes since their Green's functions do not have singularities at the unity element.

In the next section we shall derive a nice approximation $T_{\alpha, D_{11}}(\cdot, \theta)$ of the unwrapped Green's function $S_{\alpha, D_{11}}^{\infty}(\cdot, \theta)$ in closed form in both spatial and Fourier domains. The Fourier transform of this nice approximation $\hat{T}_{\alpha, D_{11}}(\cdot, \theta)$ again does not converge quickly to zero at infinity, but the difference $\hat{S}_{\alpha, D_{11}}^{\infty}(\cdot, \theta)-\hat{T}_{\alpha, D_{11}}(\cdot, \theta)$ does. Thereby one can use the approximation $\hat{T}_{\alpha, D_{11}}$ for accurate sampling of the exact Green's function by means of a discrete Fourier transform, without introducing any extra spatial isotropic diffusion. We used this to obtain Figure 5 via Figure 7.

4.3. Analytic approximations. The basis element of the generator $\left\{\tilde{A}_{i}\right\}_{i=1}^{3}$ can be approximated by

$$
\left\{\tilde{A}_{1}, \tilde{A}_{2}, \tilde{A}_{3}\right\} \approx\left\{\hat{A}_{1}, \hat{A}_{2}, \hat{A}_{3}\right\}=\left\{\partial_{\theta}, \partial_{x}+\theta \partial_{y},-\theta \partial_{x}+\partial_{y}\right\}
$$

simply by approximation $\cos \theta \approx 1$ and $\sin \theta \approx \theta$. At first glance this approximation may seem rather crude, but as we will clearly show at the end of this section, it leads to a close approximation of the true Green's function of the direction process as long as $D_{11} / \alpha$ is small. In Subsection 4.5 we derive and analyse a further improvement of this approximation using cylindrical coordinates; see also [47]. Here we explicitly derive the Green's functions (in both spatial and Fourier domains) obtained by approximation (4.36) which are (relative to the ones studied in Subsection 4.5) easier to compare with the exact solutions. The explicit form of these Green's functions in the spatial domain (for the special case $\kappa_{0}=0$ ), as will be given in Theorem 4.6, is related to the one given in [44] (without proof), where the authors incorrectly claim that this solution is the exact analytic Green's function of the direction process. By Theorem 4.6 (with explicit proof 
and derivation) we provide important insight from a group theoretical point of view. For more details concerning these analytic approximations, we refer to [13, Ch. 4.9.2].

The approximative basis elements of the generator

$$
\left\{\left(\hat{A}_{1}\right)^{2}, \hat{A}_{1}, \hat{A}_{2}, \hat{A}_{3}\right\}=\left\{\left(\partial_{\theta}\right)^{2}, \partial_{\theta}, \partial_{x}+\theta \partial_{y},-\theta \partial_{x}+\partial_{y},\right\}
$$

do generate a finite-dimensional nil-potent Lie algebra of Heisenberg type, in contrast to the Lie algebra of the true generators of the direction process (!), which is spanned by

$$
\left\{\partial_{x}, \partial_{\theta}, \partial_{y},-\theta \partial_{y},-\theta \partial_{x}, \partial_{x} \partial_{\theta}, \partial_{y} \partial_{\theta},\left(\partial_{x}\right)^{2},\left(\partial_{y}\right)^{2},\left(\partial_{\theta}\right)^{2}\right\}
$$

Using this important observation, we get

TheOREM 4.6. Let $T_{\alpha, \kappa_{0}, D_{11}}: G \rightarrow \mathbb{R}$ be the Green's function of the operator

$$
\alpha^{-1}(\alpha I-\hat{A}):=\alpha^{-1}\left(\alpha I-D_{11}\left(\hat{A}_{1}\right)^{2}+\sum_{i=1}^{3} a_{i} \hat{A}_{i}\right),
$$

with $\left(a_{1}, a_{2}, a_{3}\right)=\left(\kappa_{0}, 1,0\right)$; i.e. it is the unique solution of

$$
\left(\alpha I-D_{11}\left(\hat{A}_{1}\right)^{2}+\sum_{i=1}^{3} a_{i} \hat{A}_{i}\right) T_{\alpha, \kappa_{0}, D_{11}}=\alpha \delta_{e}
$$

which is infinitely differentiable on $G \backslash\{e\}$. It is a strictly positive function, with ${ }^{19}$

$$
\left\|T_{\alpha, \kappa_{0}, D_{11}}\right\|_{\mathbb{L}_{1}(G)} \approx\left\|T_{\alpha, \kappa_{0}, D_{11}}\right\|_{\mathbb{L}_{1}\left(\mathbb{R}^{2} \times \mathbb{R}\right)}=1
$$

for all $\alpha, D_{11}, \kappa_{0}>0$, and is given by

$$
T_{\alpha, \kappa_{0}, D_{11}}(x, y, \theta)=\alpha \frac{\sqrt{3}}{2 D_{11} \pi x^{2}} e^{-\alpha x} e^{-\frac{3(x \theta-2 y)^{2}+x^{2}\left(\theta-\kappa_{0} x\right)^{2}}{4 x^{3} D_{11}}} \mathrm{u}(x) .
$$

The Green's function of the non-integrated time process $e^{t \hat{A}} \delta_{e}$ is given by

$$
K_{t}^{\kappa_{0}, D_{11}}(x, y, \theta)=\delta(x-t) \frac{\sqrt{3}}{2 D_{11} \pi x^{2}} e^{-\frac{3(x \theta-2 y)^{2}+x^{2}\left(\theta-\kappa_{0} x\right)^{2}}{4 x^{3} D_{11}}} \mathrm{u}(x) .
$$

Proof. First we notice that by means of Hörmander's Theorem [32, Theorem 1.1, p. $149]$ the exact Green's function $S_{\alpha, \kappa_{0}, D_{11}}$ is infinitely differentiable on $G \backslash\{e\}$. This follows from the fact that the Hörmander condition is satisfied as the set

$$
\left\{\tilde{A}_{1},\left[\tilde{A}_{1}, \tilde{A}_{2}\right],\left[\tilde{A}_{1},\left[\tilde{A}_{1}, \tilde{A}_{2}\right]\right]\right\}=\left\{\partial_{\theta},\left[\partial_{\theta}, \partial_{\xi}\right],\left[\partial_{\theta},\left[\partial_{\theta}, \partial_{\xi}\right]\right]\right\}=\left\{\partial_{\theta}, \partial_{\eta}, \partial_{\xi}\right\}
$$

spans each 3-dimensional tangent space. However, for the approximation we have

$$
\left\{\hat{A}_{1},\left[\hat{A}_{1}, \hat{A}_{2}\right],\left[\hat{A}_{1},\left[\hat{A}_{1}, \hat{A}_{2}\right]\right]\right\}=\left\{\partial_{\theta},\left[\partial_{\theta}, \partial_{x}+\theta \partial_{y}\right],\left[\partial_{\theta},\left[\partial_{\theta}, \partial_{x}+\theta \partial_{y}\right]\right]\right\}=\left\{\partial_{\theta}, \partial_{y}, 0\right\}
$$

and the direction $\partial_{x}$ is missing! But by the Laplace transform over time we integrate this degeneracy out and thereby $T_{\alpha, \kappa_{0}, D_{11}}$ is (in contrast to $k_{t}^{\kappa_{0}, D_{11}}$ !) infinitely differentiable on $\hat{G} \backslash\{e\}:=\mathbb{R}^{2} \times \mathbb{R} \backslash\{0,0,0\}$.

On $\mathbb{R}^{2} \times \mathbb{R} \backslash\{0,0,0\}$ we have

$$
\left(\alpha I-D_{11}\left(\hat{A}_{1}\right)^{2}+\sum_{i=1}^{2} a_{i} \hat{A}_{i}\right) T_{\alpha, \kappa_{0}, D_{11}}=0,
$$

\footnotetext{
${ }^{19}$ The approximation is very accurate for $\frac{D_{11}}{\alpha}$ is small, which is usually the case in applications.
} 
or equivalently, again on $\hat{G} \backslash\{e\}$

$$
\partial_{x} T_{\alpha, \kappa_{0}, D_{11}}=B T_{\alpha, \kappa_{0}, D_{11}}
$$

where the generator $B=\hat{A}+\partial_{x}-\alpha I$ is given by

$$
B=\left(-\alpha I+D_{11}\left(\partial_{\theta}\right)^{2}-\theta \partial_{y}-\kappa_{0} \partial_{\theta}\right) .
$$

Moreover we have by (4.40) that

$$
\left\langle(\alpha I-\hat{A}) T_{\alpha, \kappa_{0}, D_{11}}, \phi\right\rangle=\left(T_{\alpha, \kappa_{0}, D_{11}},(\alpha I-\hat{A}) \phi\right)_{\mathbb{L}_{2}(G)}=\alpha \phi(e),
$$

for all rapidly decaying test functions $\phi \in \mathcal{S}(\hat{G})$. In particularly for $\phi=\eta \otimes \tilde{\phi}, \phi(x, y, \theta)=$ $\eta(x) \tilde{\phi}(y, \theta)$, with $\tilde{\phi} \in \mathcal{S}(\mathbb{R} \times \mathbb{R})$ arbitrary and $\eta \in \mathcal{S}(\hat{G})$ with $\eta(x)=2 \int_{x}^{\infty} G_{\epsilon}(z) \mathrm{dz}$ for $x \geq 0$, recall (3.12), which gives us by taking the limit $\epsilon \downarrow 0$ that

$$
\lim _{x \downarrow 0}\left\langle T_{\alpha, \kappa_{0}, D_{11}}(x, \cdot, \cdot), \tilde{\phi}\right\rangle=\alpha \eta(0) \tilde{\phi}(0,0)=\alpha \tilde{\phi}(0,0),
$$

for all $\tilde{\phi} \in \mathcal{S}(\mathbb{R} \times \mathbb{R})$. So we conclude that $T_{\alpha, \kappa_{0}, D_{11}}$, for $x>0$, satisfies the following evolution system:

$$
\left\{\begin{array}{l}
\partial_{x} T_{\alpha, \kappa_{0}, D_{11}}=B T_{\alpha, \kappa_{0}, D_{11}}, \\
\lim _{x \downarrow 0} T_{\alpha, \kappa_{0}, D_{11}}(x, \cdot, \cdot)=\alpha \delta_{0,0} .
\end{array}\right.
$$

Moreover, $B$ lies within the nil-potent Lie algebra spanned by ${ }^{20}$

$$
\left\{\alpha I, x \partial_{\theta}, x^{2} \partial_{y}, x \theta \partial y, x \partial_{\theta}^{2}, x^{2} \partial_{\theta} \partial_{y}, x^{3} \partial_{y}^{2}\right\} .
$$

As a result of Varadarajan (cf. [48, Theorem 3.18.11, p. 243]) or of the Campbell-BakerHausdorff formula, there exists a sequence of constants $\left\{c_{i}\right\}_{i=1}^{6}$ such that

$$
e^{x B} \delta_{e}=e^{-\alpha x} e^{-c_{6} x \partial_{\theta}} e^{-c_{5} x^{2} \partial_{y}} e^{-c_{1} x \theta \partial_{y}} e^{c_{2} x \partial_{\theta}^{2}} e^{c_{3} x^{2} \partial_{\theta} \partial_{y}} e^{c_{4} x^{3} \partial_{y}^{2}} \delta_{(0,0)}
$$

and thereby we have

$$
\begin{aligned}
& T_{\alpha, \kappa_{0}, D_{11}}(x, y, \theta)=
\end{aligned}
$$

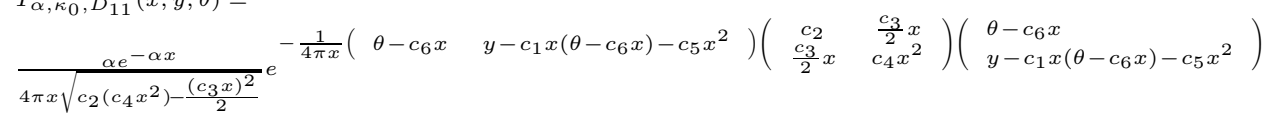

for $x>0$. Substitution of this expression in (4.47) yields

$$
c_{1}=\frac{1}{2}, c_{2}=D_{11}, \quad c_{3}=0, c_{4}=\frac{D_{11}}{12}, c_{5}=\frac{\kappa_{0}}{2}, c_{6}=\kappa_{0},
$$

which completes the proof of Theorem 4.6, where we note that (4.43) follows by (4.42) by the inverse Laplace transform.

In order to compare this approximation to the exact solution, we would like to get the Fourier transform (with respect to the spatial variables $(x, y)$ ) of the Green's function. Since this is not easily obtained by direct computation, we follow the same approach as in Subsection 4.2 for the exact solution where we unwrapped the torus.

\footnotetext{
${ }^{20}$ The basis elements all have the same physical dimension: length.
} 
Lemma 4.7. Let $\beta>0$ and let $a \in \mathbb{R}$ and let $c \in \mathbb{C}$. Then the unique (continuous) Green's function $\mathcal{G} \in C^{\infty}(\mathbb{R}, \mathbb{C})$ which satisfies

$$
\left\{\begin{array}{l}
\left(-i a \theta+\left(\partial_{\theta}\right)^{2}-c\right) \mathcal{G}=\delta_{0} \\
\mathcal{G}(\theta) \rightarrow 0 \text { as }|\theta| \rightarrow \infty
\end{array}\right.
$$

is given by $\mathcal{G}(\theta)=\frac{e^{-\sqrt{c}|\theta|}}{2 \sqrt{c}}$ for $a=0$ and for $a \neq 0$ it is given by

$$
\begin{aligned}
\mathcal{G}(\theta) & =\frac{-2 \pi}{\sqrt[3]{a i^{\operatorname{sgn}(a)-1}}}\left[\operatorname{Ai}\left(\frac{c}{(i a)^{\frac{2}{3}}} e^{\frac{i \operatorname{sgn}(a) 2 \pi}{3}}\right) \operatorname{Ai}\left(\frac{c+i a \theta}{(i a)^{\frac{2}{3}}}\right) \mathrm{u}(\theta)\right. \\
& \left.+\operatorname{Ai}\left(\frac{c}{(i a)^{\frac{2}{3}}}\right) \operatorname{Ai}\left(\frac{c+i a \theta}{(i a)^{\frac{2}{3}}} e^{\frac{i \operatorname{sgn}(a) 2 \pi}{3}}\right) \mathrm{u}(-\theta)\right], \theta \neq 0,
\end{aligned}
$$

with $\operatorname{Ai}(z)$ the Airy function of the first kind given by $\operatorname{Ai}(z)=\frac{1}{\pi} \sqrt{\frac{z}{3}} K_{1 / 3}\left(\frac{2}{3} z^{\frac{3}{2}}\right)$, where $K_{1 / 3}$ is the modified Bessel function of the second kind. Integration yields

$$
\begin{aligned}
\int_{-\infty}^{\infty} \mathcal{G}(\theta) \mathrm{d} \theta & =\frac{2 \pi}{\sqrt[3]{a^{2}(i)^{\operatorname{sgn}(a)}}}\left[\operatorname{Ai}\left(\frac{c}{(i a)^{\frac{2}{3}}}\right) e^{\frac{i \operatorname{sgn}(a) 2 \pi}{3}} I\left(\frac{c}{(i a)^{\frac{2}{3}}} e^{\frac{i \operatorname{sgn}(a) 2 \pi}{3}}\right)\right. \\
& \left.-I\left(\frac{c}{(i a)^{\frac{2}{3}}}\right) \operatorname{Ai}\left(\frac{c}{(i a)^{\frac{2}{3}}} e^{\frac{i \operatorname{sgn}(a) 2 \pi}{3}}\right)\right]
\end{aligned}
$$

where $I(z)=\int_{z}^{\infty} \operatorname{Ai}(v) \mathrm{d} v=\pi\left(\operatorname{Ai}(z) \operatorname{Gi}^{\prime}(z)-\operatorname{Ai}^{\prime}(z) \operatorname{Gi}(z)\right) ;$ cf. [1, p. 448].

Proof. We only consider the non-trivial case $a \neq 0$. It is sufficient to consider the case $c \in \mathbb{R}$, since the general case $c \in \mathbb{C}$ follows by analytic continuation. Notice with this respect that $z \mapsto \operatorname{Ai}(z)$ is an entire analytic function on $\mathbb{C}$. For $c$ real-valued we have that if $\theta \mapsto f(\theta)$ is a solution of

$$
\left(-a \theta i-\left(\partial_{\theta}\right)^{2}-c\right) f=0,
$$

then so is $\theta \mapsto \overline{f(-\theta)}$ a solution of (4.55). This is easily verified by substitution and conjugation. The general solution of (4.55) is given by

$$
f(\theta)=c_{1} \mathrm{Ai}\left(\frac{c+i a \theta}{(i a)^{\frac{2}{3}}}\right)+c_{2} \mathrm{Ai}\left(\frac{c+i a \theta}{(i a)^{\frac{2}{3}}} e^{\frac{i \operatorname{sgn}(a) 2 \pi}{3}}\right)
$$

where we notice that the Wronskian

$$
W\left[\operatorname{Ai}(z), \operatorname{Ai}\left(z e^{\frac{i \operatorname{sgn}(a) 2 \pi}{3}}\right)\right]=\frac{1}{2 \pi} e^{\operatorname{sgn}(a) \frac{\pi i}{6}} ;
$$

cf. [1, p. 446]. Furthermore, for $c$ real-valued we have

$$
\overline{\operatorname{Ai}\left(\frac{c-i a \theta}{(i a)^{\frac{2}{3}}}\right)}=\operatorname{Ai}\left(\frac{c+i a \theta}{\overline{(i a)^{\frac{2}{3}}}}\right)=\operatorname{Ai}\left(\frac{c+i a \theta}{(i a)^{\frac{2}{3}}} e^{\frac{i \operatorname{sgn}(a) 2 \pi}{3}}\right) .
$$

Since $\mathrm{Ai}\left(\frac{c+i a \theta}{(i a)^{\frac{2}{3}}}\right)$ is the only solution of (4.55) with the property $f(\theta) \rightarrow 0$ as $\theta \rightarrow \infty$, we must have

$$
\mathcal{G}(\theta)=c_{1} \operatorname{Ai}\left(\frac{c+i a \theta}{(i a)^{\frac{2}{3}}}\right) \mathrm{u}(\theta)+c_{2} \operatorname{Ai}\left(\frac{c+i a \theta}{(i a)^{\frac{2}{3}}} e^{\frac{i \operatorname{sgn}(a) 2 \pi}{3}}\right) \mathrm{u}(-\theta) .
$$


It follows by means of the Fourier transform that $\mathcal{F}[\mathcal{G}] \in \mathbb{L}_{1}(\mathbb{R})$ and as a result $\mathcal{G}$ is a continuous function vanishing at infinity, and thereby we must have $c_{1}=\lambda \operatorname{Ai}\left(\frac{c}{(i a)^{\frac{2}{3}}} e^{\frac{i \operatorname{sgn}(a) 2 \pi}{3}}\right)$ and $c_{2}=\lambda \mathrm{Ai}\left(\frac{c}{(i a)^{\frac{2}{3}}}\right)$, for some $0 \neq \lambda \in \mathbb{C}$, which we again determine by means of substitution of (4.57) in (4.55) yielding

$$
\lambda(i a)^{\frac{1}{3}}\left[\operatorname{Ai}\left(\frac{c}{(i a)^{\frac{2}{3}}} e^{\frac{i \operatorname{sgn}(a) 2 \pi}{3}}\right) \operatorname{Ai}^{\prime}\left(\frac{c}{(i a)^{\frac{2}{3}}}\right)-\operatorname{Ai}^{\prime}\left(\frac{c}{(i a)^{\frac{2}{3}}} e^{\frac{i \operatorname{sgn}(a) 2 \pi}{3}}\right) \operatorname{Ai}\left(\frac{c}{(i a)^{\frac{2}{3}}}\right)\right] \delta_{0}=\delta_{0},
$$

from which we deduce together with $(4.56)$ that $\lambda=\frac{2 \pi}{\sqrt[3]{a i(\operatorname{sgn}(a)-1)}}$. Finally we notice that (4.54) follows by direct computation where we notice that $z \mapsto \operatorname{Ai}(z)$ is entire analytic, which allows us to change the path of integration.

By setting $a=\frac{w_{y}}{D_{11}}$ and $c=\frac{i w_{x}+\alpha}{D_{11}}$ in Lemma 4.7, we obtain the following result, which is similar to Theorem 4.5 and enables us to compare the exact solution $S_{\alpha, D_{11}}^{\infty}$ with its Heisenberg approximation $T_{\alpha, D_{11}}$ via the Fourier domain.

ThEOREM 4.8. The solution $T_{\alpha, D_{11}}: \mathbb{R}^{3} \backslash\{0,0,0\}$ of the problem

$$
\left\{\begin{array}{l}
\left(\partial_{x}+\theta \partial_{y}-D_{11}\left(\partial_{\theta}\right)^{2}+\alpha\right) T_{\alpha, D_{11}}=\alpha \delta_{e}, \\
T_{\alpha, D_{11}}(\cdot, \cdot, \theta) \rightarrow 0 \text { uniformly on compacta as }|\theta| \rightarrow \infty \\
T_{\alpha, D_{11}} \in \mathbb{L}_{1}\left(\mathbb{R}^{3}\right)
\end{array}\right.
$$

is given by $T_{\alpha, D_{11}}(x, y, \theta)=\mathcal{F}^{-1}\left[\left(\omega_{x}, \omega_{y}\right) \mapsto \hat{T}_{\alpha, D_{11}}\left(\omega_{x}, \omega_{y}, \theta\right)\right](x, y)$ where $\hat{T}_{\alpha, D_{11}} \in$ $C(\mathbb{R}, \mathbb{C})$ is given by

$$
\begin{aligned}
\hat{T}_{\alpha, D_{11}}\left(\omega_{x}, \omega_{y}, \theta\right)= & \frac{\alpha}{D_{11}} \sqrt[3]{\frac{D_{11}}{\omega_{y} i^{\operatorname{sgn}\left(\omega_{y}\right)-1}}} \\
& \times\left[\operatorname{Ai}\left(\frac{1}{\sqrt[3]{D_{11}}} \frac{i w_{x}+\alpha}{\left(i \omega_{y}\right)^{\frac{2}{3}}} e^{\frac{i \operatorname{sgn}\left(\omega_{y}\right) 2 \pi}{3}}\right) \operatorname{Ai}\left(\frac{1}{\sqrt[3]{D_{11}}} \frac{i \omega_{x}+\alpha+i \omega_{y} \theta}{\left(i \omega_{y}\right)^{\frac{2}{3}}}\right) \mathrm{u}(\theta)\right. \\
+ & \left.\operatorname{Ai}\left(\frac{1}{\sqrt[3]{D_{11}}} \frac{i w_{x}+\alpha}{\left(i w_{y}\right)^{\frac{2}{3}}}\right) \operatorname{Ai}\left(\frac{1}{\sqrt[3]{D_{11}}} \frac{i w_{x}+\alpha+i \omega_{y} \theta}{\left(i w_{y}\right)^{\frac{2}{3}}} e^{\frac{i \operatorname{sgn}\left(w_{y}\right) 2 \pi}{3}}\right) \mathrm{u}(-\theta)\right]
\end{aligned}
$$

which holds for $\omega_{y} \neq 0$, and for $\omega_{y}=0$ we have

$$
\hat{T}_{\alpha, D_{11}}\left(\omega_{x}, 0, \theta\right)=\frac{1}{4 \pi} \sqrt{\frac{\alpha}{D_{11}}}\left(\alpha^{-1} i \omega_{x}+1\right)^{-1 / 2} e^{-\sqrt{\frac{i \omega_{x}+\alpha}{D_{11}}}|\theta|} .
$$

REMARK 4.9. By means of straightforward computation and an asymptotic expansion of the Airy function (see [1, p. 448, formula 10.4.59]),

$$
\operatorname{Ai}(z) \sim \frac{1}{2 \sqrt{\pi}} z^{-\frac{1}{4}} e^{-\xi} \sum_{k=0}^{\infty}(-1)^{k} c_{k} \xi^{-k} \text { with } \xi=\frac{2}{3} z^{\frac{3}{2}}, c_{k}=\frac{\Gamma\left(3 k+\frac{1}{2}\right)}{54^{k} k ! \Gamma\left(k+\frac{1}{2}\right)},|\arg (z)|<\pi,
$$

it follows that $\hat{T}_{\alpha, D_{11}}$ is everywhere continuous, since

$$
\lim _{\omega_{y} \downarrow 0} \hat{T}_{\alpha, D_{11}}\left(\omega_{x}, \omega_{y}, \theta\right)=\frac{1}{4 \pi} \sqrt{\frac{\alpha}{D_{11}}}\left(\alpha^{-1} i \omega_{x}+1\right)^{-1 / 2} e^{-\sqrt{\frac{i \omega_{x}+\alpha}{D_{11}}}|\theta|}=\hat{T}_{\alpha, D_{11}}\left(\omega_{x}, 0, \theta\right),
$$


with in particular

$$
\begin{aligned}
\hat{T}_{\alpha, D_{11}}\left(0,0, \theta_{1}\right) & =\frac{1}{2 \pi}\left\|T\left(\cdot, \cdot, \theta_{1}\right)\right\|_{\mathbb{L}_{1}\left(\mathbb{R}^{2}\right)} \\
& =\frac{1}{2 \pi} \int_{0}^{\infty} \int_{-\infty}^{\infty} T_{\alpha, D_{11}}\left(x, y, \theta_{1}\right) \mathrm{dxdy}=\frac{1}{4 \pi} \sqrt{\frac{\alpha}{D_{11}}} e^{-\sqrt{\frac{\alpha}{D_{11}}}\left|\theta_{1}\right|},
\end{aligned}
$$

which is the probability density that a random walker (with initial orientation $\theta=0$ ) of the approximative direction process stays in the plane $\theta=\theta_{1}$. Notice that the larger $D_{11}$, the smaller the probability density that the random walker stays within the plane $\theta=0$, and also the larger its expected lifetime $E(T)=(1 / \alpha)$, the smaller the probability that the oriented particle remains in the plane $\theta=0$.

See Figure 3 for an illustration of the quality of the approximation in the Fourier domain. Figure 4 shows the difference between the exact periodic Green's function $S_{\alpha, D_{11}}$ and the exact Green's function with infinite boundaries. It shows that (4.27) can be truncated at low $N$. See Figure 5 for an illustration of the exact Green's function and its marginals in the spatial domain. For comparison between the Heisenberg approximation $T_{\alpha, D_{11}}$ and the exact Green's function with infinite boundaries, see Figure 6 . Note that this figure also includes the case $\kappa_{0}>0$, which will be studied in Subsection 4.6. To get an idea of where the approximative Green's function $T_{\alpha, D_{11}, \kappa_{0}}$ approximates the exact Green's function best, see Figure 7.

These figures show that for $D_{11} / \alpha>0$ (and $\kappa_{0}$ ) sufficiently small, $T_{\alpha, D_{11}}$ is a good approximation of $S_{\alpha, D_{11}}^{\infty}$, which is (for $D_{11}>0$ reasonably small) extremely close (differences can be neglected) to a periodic function in $\theta$. Nevertheless for large $D_{11} / \alpha$, say $D_{11} / \alpha>5$, the tails of the Green's functions behave differently, which is to be expected, as in the Heisenberg-type approximation the random walker must progress in the positive $x$ direction $x>0$, whereas in the exact case random walkers are allowed to turn around in the negative $x$ direction (although this is very unlikely); see Figure 4 and Figure 6 . In the Heisenberg-type approximation the traveling time of the unit speed random walker is negatively exponentially distributed along the $x$-axis, whereas in the exact case the unit speed random walker is negatively exponentially distributed along its path. If $D_{11} / \alpha$ is small, the total length of the path is close to the length of its projection on the $x$-axis.

4.4. Computation of completion fields. The concept of a completion field is well known in image analysis; see for example [44], [51], [6], [15]. The idea is simple: Consider two left-invariant stochastic processes on the Euclidean motion group, one with forward convection, say its forward Kolmogorov equation is generated by $A$, and one with the same stochastic behavior but with backward convection, i.e. its forward Kolmogorov equation is generated by the adjoint of $A^{*}$ of $A$. Then we compute the probability density that the random walkers from both stochastic processes collide, which equals

$$
\Phi(U)=(A-\alpha I)^{-1} U\left(A^{*}-\alpha I\right)^{-1} W, \quad U, W \in \mathbb{L}_{2}(G) \cap \mathbb{L}_{1}(G),
$$

where $U, W$ are initial distributions. This collision probability is called a completion field as it serves as a model for perceptual organization in the sense that elongated local image fragments are completed in a more global coherent structure. These initial distributions can for example be obtained from an image by means of a well-posed invertible wavelet transform constructed by a reducible representation of the Euclidean motion group as 

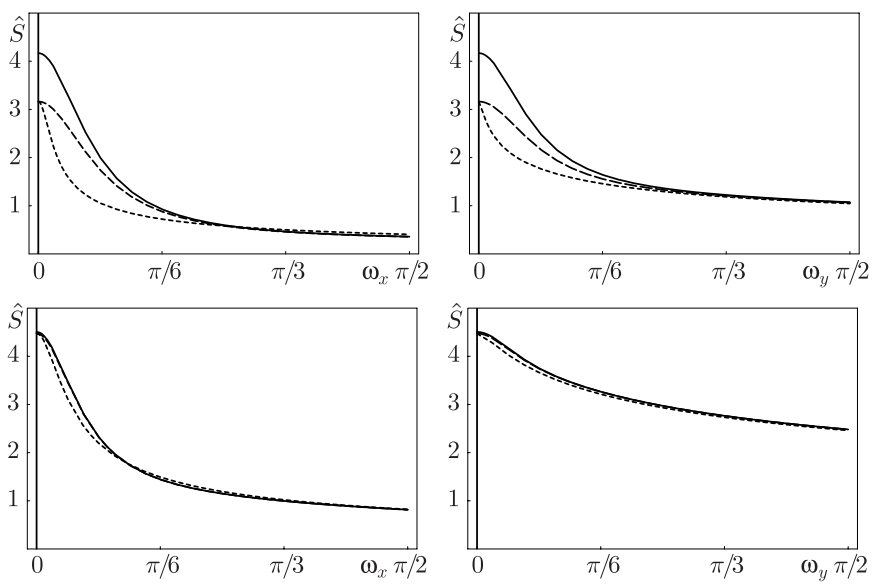

FIG. 3. Top row: The solid line denotes the real component of the analytic Green's function $\hat{S}_{\alpha, D_{11}}\left(\omega_{x}, \omega_{y}, 0\right)$ for $\alpha=1 / 20, D_{11}=1 / 2$ and $a_{2}=1$ along the $\omega_{x}$-axis on the left and along the $\omega_{y}$-axis on the right. The line with long dashes represents the real component of $\hat{S}_{\alpha, D_{11}, D_{22}, D_{33}}^{\infty, a_{1}, a_{2}, a_{3}}\left(\omega_{x}, \omega_{y}, 0\right)$. The line with short dashes stands for the approximation $\hat{T}_{\alpha, D_{11}}\left(\omega_{x}, \omega_{y}, 0\right)$, where we recall (4.59). For these extreme parameter settings the approximations are relatively poor; see also Figure 4. Bottom row: Same settings as top row, but now for $D_{11}=1 / 2$ and $\alpha=1 / 10$. Note that the smaller $D_{11} / \alpha$, the better the approximations.

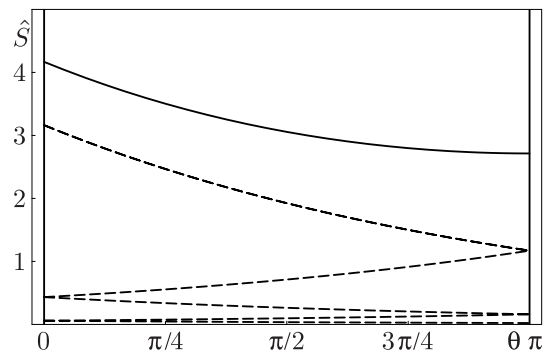

FIG. 4. The solid line denotes the real part of the Fourier transform of the true Green's function $\hat{S}_{\alpha, D_{11}}(0,0, \theta)$ for $\alpha=1 / 20, D_{11}=1 / 2$ and $a_{2}=1$ along the $\theta$-axis. The dashed line represents the real component of $\hat{S}_{\alpha, D_{11}}^{\infty}(0,0, \theta)$ and equivalently $\hat{T}_{\alpha, D_{11}}(0,0, \theta)$ with their components outside the $\theta$-interval $[-\pi, \pi]$ mapped back onto the torus domain to ensure the periodic boundary condition at $\theta= \pm \pi$. In this extreme case the series (4.27) can be truncated at $N=4$ to obtain a close approximation.

explained in [15]. Alternatives are lifting using the interesting framework of curve indicator random fields [5] or (more ad hoc) by putting a limited set of delta distributions after tresholding some end-point detector or putting them simply by hand [51]. Here we do not go into detail on how these initial distributions can be obtained, but we only 


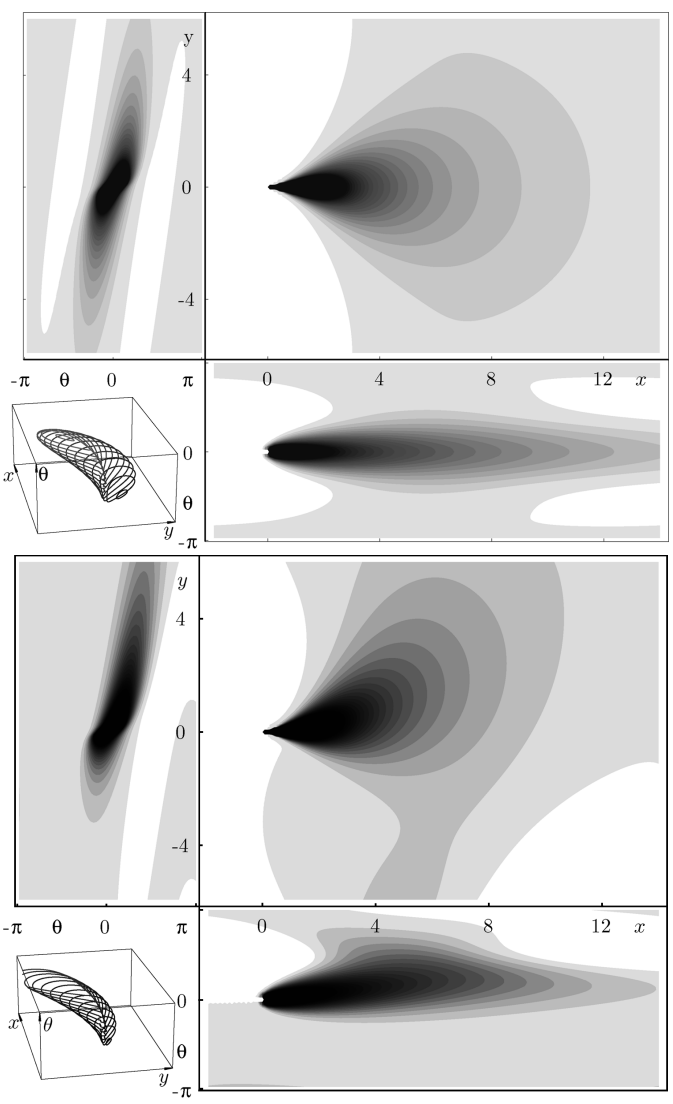

FIG. 5. Top: Contour plots of the marginals (obtained from $S_{\alpha, \kappa_{0}, D_{11}}$ by integration over $x, \theta$ and $y$ ) of the exact Green's function $S_{\alpha, \kappa_{0}, D_{11}}$ of the direction process $\left(\kappa_{0}=0, a_{2}=1, D_{11}=\right.$ $\left.\frac{1}{32}, \alpha=\frac{1}{10}\right)$. Bottom: Same settings $\left(a_{1}=1, D_{11}=\frac{1}{32}, \alpha=\frac{1}{10}\right)$ but now including a positive angular drift $a_{1}=\kappa_{0}=\frac{1}{10}$. The other parameters $a_{3}, D_{22}, D_{33}$ have been put to zero. In the lower left corners of both figures: Contour plot of $S_{\alpha=\frac{1}{10}, \kappa_{0}, D_{11}=\frac{1}{32}}$ in $\mathbb{R}^{2} \rtimes \mathbb{T}$.

consider the case $U=\delta_{\left(0,0, \theta_{0}\right)}$ and $W=\delta_{\left(x_{1}, y_{1}, \theta_{1}\right)}, x_{1}, y_{1} \in \mathbb{R}$. In this case we obtain by means of Theorem 4.6 the following approximations of the completion fields:

$$
\begin{aligned}
\hat{C}_{g_{0}, g_{1}}^{\alpha, D_{11}, \kappa_{0}, \kappa_{1}}(\mathbf{x}, \theta) & =\alpha^{2}\left((\hat{A}-\alpha I)^{-1} \delta_{\mathbf{x}_{0}, \theta_{0}}\right)(\mathbf{x}, \theta)\left(\left(\hat{A}^{*}-\alpha I\right)^{-1} \delta_{\mathbf{x}_{1},-\theta_{1}}\right)(\mathbf{x}, \theta) \\
& =T_{\alpha, \kappa_{0}, D_{11} ; \mathbf{x}_{0}, \theta_{0}}(x, y, \theta) T_{\alpha, \kappa_{1}, D_{11} ;-x_{1}, y_{1}, \theta_{1}}(-x, y,-\theta),
\end{aligned}
$$

with corresponding modes, obtained by solving for

$$
\left\{\begin{array}{l}
\partial_{y}\left\{T_{\alpha, \kappa_{0}, D_{11} ; \mathbf{0}, \theta_{0}}(x, y, \theta) T_{\alpha, \kappa_{1}, D_{11} ;-x_{1}, y_{1}, \theta_{1}}(-x, y,-\theta)\right\}=0 \\
\partial_{\theta}\left\{T_{\alpha, \kappa_{0}, D_{11} ; \mathbf{0}, \theta_{0}}(x, y, \theta) T_{\alpha, \kappa_{1}, D_{11} ;-x_{1}, y_{1}, \theta_{1}}(-x, y,-\theta)\right\}=0
\end{array}\right.
$$



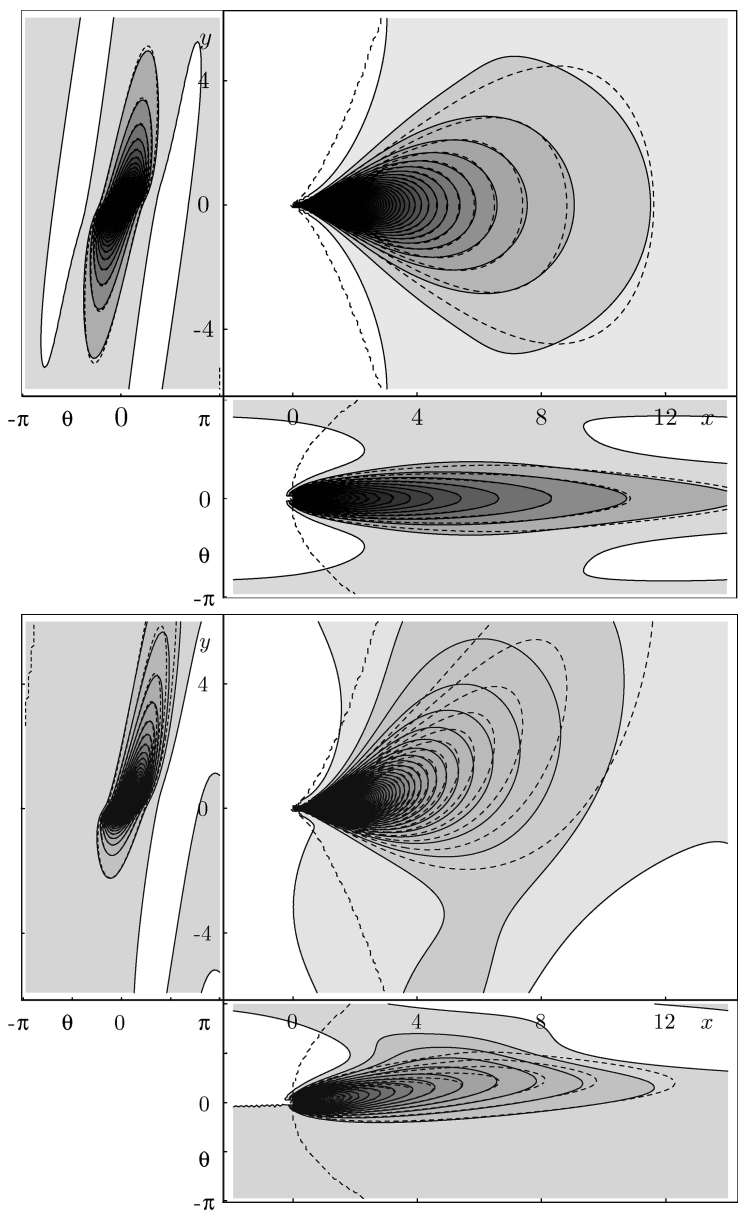

FIG. 6. A comparison of the level curves of the marginals of $T_{\alpha, \kappa_{0}, D_{11}}$ and $S_{\alpha, \kappa_{0}, D_{11}}^{\infty}$, for $\alpha=\frac{1}{10}, D_{11}=\frac{1}{32}$. Top row, $\kappa_{0}=0$. Bottom row, $\kappa_{0}=0.1$. Dashed lines denote the level sets of the Heisenberg approximation $T_{\alpha, \kappa_{0}, D_{11}}$. Note that the difference is very small. Nevertheless, the difference is best seen in the iso-contours close to zero. For example for $\kappa_{0}=0$ in the exact case there is a very small probability that the random walker turns around, whereas in the approximate case the random walker must move forward.

These modes depend only on the difference $\kappa_{0}-\kappa_{1}$ but not on $D_{11}$ nor on $\alpha$ :

$$
\begin{aligned}
y(x) & =x \theta_{0}+\frac{x^{3}}{x_{1}^{3}}\left(-2 y_{1}+x_{1}\left(\theta_{0}-\theta_{1}\right)\right)+\frac{x^{2}}{x_{1}^{2}}\left(3 y_{1}+x_{1}\left(\theta_{1}-2 \theta_{0}\right)\right) \\
& +\frac{\left(\kappa_{0}-\kappa_{1}\right)}{x_{1}^{3}}\left(x-x_{1}\right)^{2}\left(\frac{x_{1}}{2}-x\right) x^{2}, \\
\theta(x) & =\theta_{0}+2 \frac{x}{x_{1}^{2}}\left(3 y_{1}+x_{1}\left(\theta_{1}-2 \theta_{0}\right)\right)-3 \frac{x^{2}}{x_{1}^{3}}\left(2 y_{1}+x_{1}\left(\theta_{1}-\theta_{0}\right)\right) \\
& +\frac{\left(\kappa_{0}-\kappa_{1}\right)}{x_{1}^{3}} x\left(x-x_{1}\right)\left(-3 x^{2}+3 x_{1} x-x_{1}^{2}\right),
\end{aligned}
$$

where $x \in\left[0, x_{1}\right]$ and $y(0)=0, \theta(0)=\theta_{0}$ and $y\left(x_{1}\right)=y_{1}, \theta\left(x_{1}\right)=-\theta_{1}$ and $\frac{d y}{d x}(0)=$ $\theta_{0}$ and $\frac{d y}{d x}\left(x_{1}\right)=-\theta_{1}$; see Figure 8 . These modes are the unique minimizers of the 


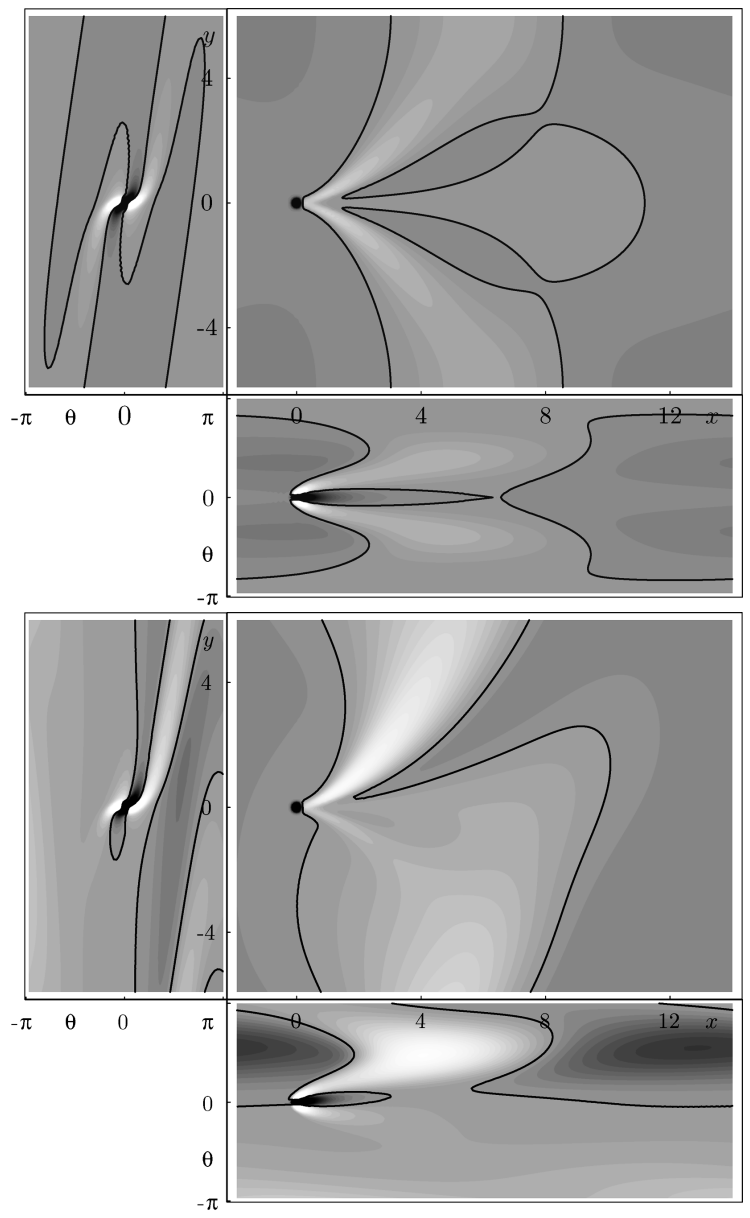

FIG. 7. Contour plots of the marginals of the difference $S_{\alpha, \kappa_{0}, D_{11}}-$ $T_{\alpha, \kappa_{0}, D_{11}}$ of the exact Green's function $S_{\alpha, \kappa_{0}, D_{11}}$ and its Heisenberg approximation $T_{\alpha, \kappa_{0}, D_{11}}$ of the direction process $\left(a_{2}=1, D_{11}=\right.$ $\frac{1}{32}, \alpha=\frac{1}{10}$ ), without angular drift $\kappa_{0}=0$ (top figure) and with angular drift $\kappa_{0}=0.2$ (bottom figure). Same parameter settings as Figure 5. The black lines show where $T_{\alpha, \kappa_{0}, D_{11}}$ and $S_{\alpha, \kappa_{0}, D_{11}}$ are equal. The whiter areas are the parts where the exact solution $S_{\alpha, \kappa_{0}, D_{11}}$ is larger than the approximation $T_{\alpha, \kappa_{0}, D_{11}}$.

following variational problem:

$$
\operatorname{argmin}\left\{\mathcal{E}(y)=\int_{0}^{x_{1}}\left(y^{\prime \prime}(x)-\frac{\left(\kappa_{1}-\kappa_{0}\right) c(x)}{x_{1}^{3}}\right)^{2} \mathrm{~d} x \mid y(0)=0, y\left(x_{1}\right)=y_{1}, y^{\prime}(0)=\theta_{0}, y^{\prime}\left(x_{1}\right)=-\theta_{1}\right\}
$$

where $c(x)=20\left(x-x_{1}\right)\left(x-\frac{x_{1}}{2}\right) x$ and $y^{\prime}(x)=\theta(x)+\frac{\left(\kappa_{1}-\kappa_{0}\right) d(x)}{x_{1}^{3}}$ with

$$
d(x)=-2 x^{2}\left(x-x_{1}\right)^{2} .
$$


The variational problem (4.62) for the case $\kappa_{0}=\kappa_{1}$ is indeed the corresponding (with arclength replaced by $x$ ) approximation of the elastica functional in [40] and indeed $\frac{\partial E}{\partial v}(y)=0$ for all $v \in \mathcal{D}\left(\left(0, x_{1}\right)\right)$ if and only if $y^{(4)}(x)=\left(\kappa_{1}-\kappa_{0}\right) c^{(2)}(x)$ under the conditions $y(0)=0, y\left(x_{1}\right)=y_{1}, y^{\prime}(0)=\theta_{0}, y^{\prime}\left(x_{1}\right)=-\theta_{1}$.

We note that because of left-invariance with respect to the 5 -dimensional Heisenbergtype of group, we have

$$
\hat{S}_{\alpha, \kappa_{0}, D_{11} ; \mathbf{x}^{\prime}, \theta^{\prime}}(x, y, \theta)=\hat{S}_{\alpha, \kappa_{0}, D_{11} ; e}\left(x-x^{\prime}, y-y^{\prime}-\theta^{\prime}\left(x-x^{\prime}\right), \theta-\theta^{\prime}\right) .
$$

As a result the approximate completion field (and thereby its mode) is not left-invariant on $\mathbb{R}^{2} \rtimes \mathbb{T}$ and thereby its marginal is not Euclidean invariant. As a result the formulas do depend ${ }^{21}$ on the choice of coordinate system $\{x, y\}$.

However, this problem does not arise for the exact completion field

$$
C^{g_{0}, g_{1}, \alpha, D_{11}, \kappa_{0}, \kappa_{1}}=\alpha^{2}\left((A-\alpha I)^{-1} \delta_{\mathbf{x}_{0}, \theta_{0}}\right)\left(\left(A^{*}-\alpha I\right)^{-1} \delta_{\mathbf{x}_{1}, \theta_{1}}\right),
$$

since by left-invariance of the generator $A$ we have

$$
(A-\alpha I)^{-1} \delta_{g_{0}}=(A-\alpha I)^{-1} \mathcal{L}_{g_{0}} \delta_{e}=\mathcal{L}_{g_{0}}(A-\alpha I)^{-1} \delta_{e}
$$

and therefore

$$
C^{h g_{0}, h g_{1}, \alpha, D_{11}, \kappa_{0}, \kappa_{1}}=\mathcal{L}_{h} C^{g_{0}, g_{1}, \alpha, D_{11}, \kappa_{0}, \kappa_{1}}
$$

for all $h \in \mathbb{R}^{2} \rtimes \mathbb{T}$. Based on the results by Mumford [40, p. 496] we would conjecture that the elastica curves which minimize $\int \frac{\kappa(s)^{2}}{4 D_{11}}+\alpha \mathrm{d} s$ and which satisfy $2 \kappa^{\prime \prime}(s)+\kappa^{3}(s)=$ $4 \alpha D_{11} \kappa(s)$, with $s$ the arc-length parameter, are the unique curves along which

$$
\left\{\begin{array}{l}
\partial_{\eta} C_{\alpha, D_{11}, \kappa_{0}=\kappa_{1}=0}^{g_{0}, g_{1}}=\left(-\sin \theta \partial_{x}+\cos \theta \partial_{y}\right) C_{\alpha, D_{11}, \kappa_{0}=\kappa_{1}=0}^{g_{0}, g_{1}}=0, \\
\partial_{\theta} C_{\alpha, D_{11}, \kappa_{0}=\kappa_{1}=0}^{g_{0}, g_{1}}=0,
\end{array}\right.
$$

despite the fact that the system $\left\{\partial_{\theta}, \partial_{\eta}\right\}$ (in contrast to $\left\{\partial_{\theta}, \partial_{y}\right\}$ ) is not an integrable system of vector fields.

4.5. Better analytic approximations of the Green's function of the direction process. In this subsection we derive an improvement of the analytic approximation derived in Subsection 4.3 (especially for the cases $\kappa_{0}>0$ ) by making use of cylindrical coordinates. We first express the left-invariant vector fields on $\mathbb{R}^{2} \rtimes \mathbb{T}$ in cylindrical coordinates:

$$
\left\{\begin{array}{l}
\tilde{A}_{1}=\partial_{\theta} \\
\tilde{A}_{2}=\cos (\theta-\phi) \partial_{r}+\frac{1}{r} \sin (\theta-\phi) \partial_{\phi} \\
\tilde{A}_{3}=-\sin (\theta-\phi) \partial_{r}+\frac{1}{r} \cos (\theta-\phi) \partial_{\phi}
\end{array}\right.
$$

which enables us to write the objective equation

$$
-\left(D_{11}\left(\tilde{A}_{1}\right)^{2}-\sum_{i=1}^{3} \alpha_{i} \tilde{A}_{i}-\alpha I\right) S_{\alpha,\left\{a_{i}\right\}, D_{11}}=\alpha \delta_{e},
$$

with $a_{1}=\kappa_{0}, a_{2}=1, a_{3}=0$, in cylindrical coordinates:

$$
\left(\cos (\theta-\phi) \partial_{r}+\frac{1}{r} \sin (\theta-\phi) \partial_{\phi}+\kappa_{0} \partial_{\theta}-D_{11}\left(\partial_{\theta}\right)^{2}+\alpha I\right) S_{\alpha, \kappa_{0}, D_{11}}=\alpha \delta_{e} .
$$

${ }^{21}$ If $\{x, y\}$ is aligned with $g_{0}$, the result is different than if it is (slightly) aligned with $g_{1}$. 

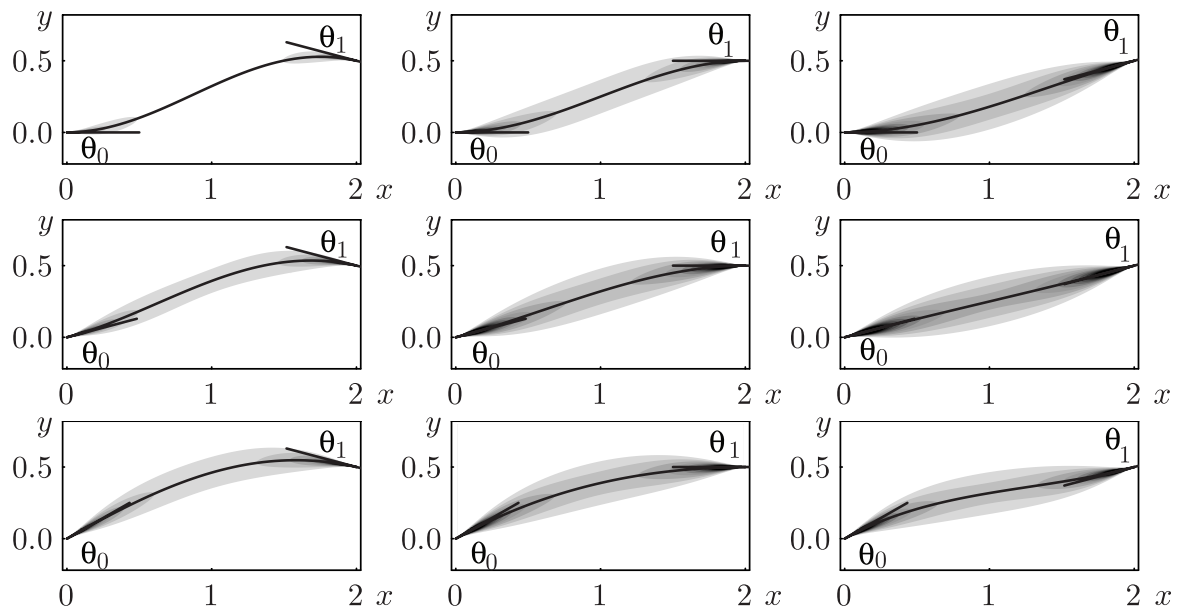

FIG. 8. The shading in these plots denotes the marginal of the analytic completion field approximation (4.60) obtained via integration over $\theta$ for $x \in(0,2), y \in(-0.2,0.8)$, i.e.

$$
\int_{\mathbb{R}} T_{D_{11}=0.5, \theta_{0}=2, \kappa_{0}=0}^{x_{0}=0, y_{0}=0, \theta_{0}}(x, y, \theta) T_{D_{11}=0.5, \theta_{1}=2, \kappa_{1}=0}^{x_{1}=2, y_{1}=0.5, \theta_{1}}(-x, y,-\theta) \mathrm{d} \theta
$$

for $\theta_{0}=0^{\circ}, 15^{\circ}, 30^{\circ}$ from top to bottom and for $\theta_{1}=-15^{\circ}, 0^{\circ}, 15^{\circ}$ from left to right. The lines drawn on top of these completion fields are the modes (4.61), the optimal connecting lines.

By approximating $\cos (\theta-\phi) \approx 1$ and $\sin (\theta-\phi) \approx(\theta-\phi)$, we get the following approximations of the left-invariant generators (4.63):

$$
\breve{A}_{1}=\partial_{\theta}, \quad \breve{A}_{2}=\partial_{r}+(\theta-\phi) \frac{1}{r} \partial_{\phi}, \quad \breve{A}_{3}=-(\theta-\phi) \partial_{r}+\frac{1}{r} \partial_{\phi},
$$

and we obtain the following equation for the approximation $\breve{T}_{\alpha, \kappa_{0}, D_{11}}$ of the Green's function $S_{\alpha, D_{11}}$ (or rather $S_{\alpha, D_{11}}^{\infty}$ ) of the resolvent of the forward Kolmogorov equation:

$$
\left(1 \partial_{r}+\frac{1}{r}(\theta-\phi) \partial_{\phi}+\kappa_{0} \partial_{\theta}-D_{11}\left(\partial_{\theta}\right)^{2}+\alpha I\right) \breve{T}_{\alpha, \kappa_{0}, D_{11}}=\alpha \delta_{e}
$$

Notice that the approximation $\breve{T}_{\alpha, \kappa_{0}, D_{11}} \approx S_{\alpha, \kappa_{0}, D_{11}}^{\infty}$ is better than the approximation $T_{\alpha, \kappa_{0}, D_{11}} \approx S_{\alpha, \kappa_{0}, D_{11}}^{\infty}$. For high angular drifts in particular, see Figure 9. The characteristics corresponding to $\breve{T}_{\alpha, \kappa_{0}, D_{11}}$ are given by

$$
\left\{\begin{array}{l}
\dot{\theta}=\kappa_{0}, \\
\dot{\phi}=\frac{1}{r}(\theta-\phi), \Rightarrow\left\{\begin{array}{l}
x(s)=s \cos \left(\frac{\kappa_{0} s}{2}\right) \\
\dot{r}=1
\end{array},\right. \\
\theta(s)=s \sin \left(\frac{\kappa_{0} s}{2}\right)
\end{array}\right.
$$

The substitution

$$
\left\{\begin{array} { l } 
{ v ( r ) = r , } \\
{ w ( r ) = r \phi , }
\end{array} \Rightarrow \left\{\begin{array}{l}
\frac{d}{d r} \breve{T}_{\alpha, \kappa_{0}, D_{11}}=\left.\left(1 \frac{d}{d v}+\phi \frac{d}{d w}\right) \breve{\mathcal{T}}_{\alpha, \kappa_{0}, D_{11}}(w, v, \theta)\right|_{w=r \phi, v=r} \\
\frac{d}{d \phi} \breve{\mathcal{T}}_{\alpha, \kappa_{0}, D_{11}}=\left.r \frac{d}{d w} \breve{\mathcal{T}}_{\alpha, \kappa_{0}, D_{11}}(w, v, \theta)\right|_{w=r \phi, v=r}
\end{array}\right.\right.
$$



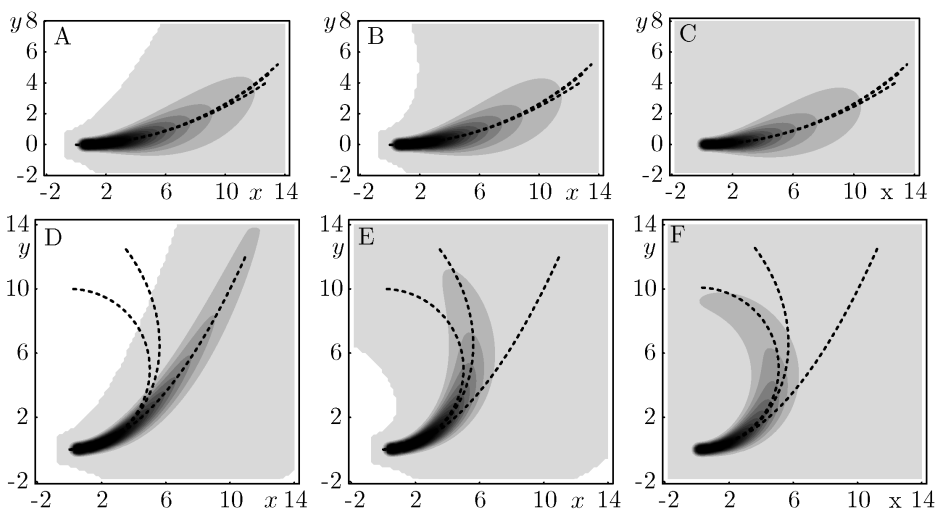

FIG. 9. A comparison between the $x y$-marginals of the exact solution $S_{\alpha, \kappa_{0}, D_{11}}$ of the direction process with angular drift (bottom row, $a_{1}=\kappa_{0}=0.2$; top row, $\left.\kappa_{0}=0.05\right)$, with $D_{11}=\frac{\sigma^{2}}{2}, \sigma=0.1$, $\left(\left(a_{2}, a_{3}\right)=(1,0), D_{33}=D_{22}=0\right)$ (see C, F) and the $x y$-marginal of the Cartesian $T_{\alpha, \kappa_{0}, D_{11}}$ (see A, D) and polar $\breve{T}_{\alpha, \kappa_{0}, D_{11}}$ (see B, E) approximations. Note that

$$
\int_{\mathbb{R}} \breve{T}_{\alpha, \kappa_{0}, D_{11}}(r, \phi, \theta) \mathrm{d} \theta=\frac{\sqrt{3 \pi} \alpha}{2 \sqrt{D_{11}} \pi r^{\frac{3}{2}}} e^{-\alpha r-\frac{3\left(r \kappa_{0}-2 \phi\right)^{2}}{16 D_{11}}}
$$

and

$\int_{\mathbb{R}} T_{\alpha, \kappa_{0}, D_{11}}(x, y, \theta) \mathrm{d} \theta=\frac{1}{2} \alpha D_{11} \sqrt{\frac{3}{\pi}}\left(\frac{1}{D_{11} x}\right)^{3 / 2} e^{-x \alpha-\frac{3\left(-2 y+\kappa_{0} x^{2}\right)^{2}}{16 D_{11} x^{3}}}$.

For comparison the corresponding exponential curves

$t \mapsto\left(\kappa_{0}^{-1} \sin \kappa_{0} t, \kappa_{0}^{-1}\left(1-\cos \kappa_{0} t\right), \kappa_{0} t\right), t \mapsto\left(t, \frac{\kappa_{0}}{2} t^{2}, \kappa_{0} t\right), t \mapsto\left(t \cos \left(\frac{\kappa_{0} t}{2}\right)\right.$,

$\left.t \sin \left(\frac{\kappa_{0} t}{2}\right), \kappa_{0} t\right)$ are plotted on top. So if $\kappa_{0}>0$, the Heisenberg approximation in polar coordinates yields a better approximation.

with $\breve{\mathcal{T}}_{\alpha, \kappa_{0}, D_{11}}(r, r \phi, \theta)=\breve{T}_{\alpha, \kappa_{0}, D_{11}}(r \cos \phi, r \sin \phi, \theta)$ gives

$$
\left(\partial_{v}+\theta \partial_{w}+\kappa_{0} \partial_{\theta}-D_{11} \partial_{\theta}^{2}+\alpha\right) \breve{T}_{\alpha, \kappa_{0}, D_{11}}=\alpha \delta_{e}
$$

and thereby by means of Theorem 4.6 we obtain the solution:

$$
\breve{T}_{\alpha, \kappa_{0}, D_{11}}(r, \phi, \theta)=T_{\alpha, \kappa_{0}, D_{11}}(r, r \phi, \theta)=\alpha \frac{\sqrt{3}}{2 \pi D_{11} r^{2}} e^{-\alpha r} e^{-\frac{3(\theta-2 \phi)^{2}+\left(\theta-\kappa_{0} r\right)^{2}}{4 r D 11}} .
$$

4.6. The generalized direction process: The cases $a_{1}=\kappa_{0} \geq 0, a_{2} \neq 0, a_{3}=0$, $D_{11}>0, D_{22}=D_{33}>0$. Consider the case $a_{1}=\kappa_{0} \geq 0, a_{2} \neq 0, a_{3}=0, D_{11}>0$, $D_{22}=D_{33}>0$. This means that we add extra isotropic diffusion and an angular drift $\kappa_{0} \geq 0$ into the direction process. Again we consider $\theta \in \mathbb{R}$ with the boundary condition that solutions must uniformly vanish as $r=\sqrt{x^{2}+y^{2}} \rightarrow \infty$.

TheOREM 4.10. The solution $S_{\alpha, D_{11}}^{\infty}: \mathbb{R}^{3} \backslash\{0,0,0\}$ of the problem

$$
\left\{\begin{array}{l}
\left(\partial_{\xi}+\kappa_{0} \partial_{\theta}-D_{11}\left(\partial_{\theta}\right)^{2}-D_{22}\left(\partial_{\eta}\right)^{2}-D_{22}\left(\partial_{\xi}\right)^{2}+\alpha I\right) S_{\alpha, D_{11}}^{\infty}=\alpha \delta_{e} \\
S_{\alpha, D_{11}}^{\infty}(\cdot, \cdot, \theta) \rightarrow 0 \text { uniformly on compacta as }|\theta| \rightarrow \infty \\
S_{\alpha, D_{11}}^{\infty} \in \mathbb{L}_{1}\left(\mathbb{R}^{3}\right)
\end{array}\right.
$$


is given by $S_{\alpha, D_{11}, D_{22}, \kappa_{0}}^{\infty}(x, y, \theta)=\mathcal{F}^{-1}\left[\left(\omega_{x}, \omega_{y}\right) \mapsto \hat{S}_{\alpha, D_{11}, D_{22}, \kappa_{0}}^{\infty}\left(\omega_{x}, \omega_{y}, \theta\right)\right](x, y)$ where

$$
\begin{aligned}
& \hat{S}_{\alpha, D_{11}, D_{22}, \kappa_{0}}^{\infty}\left(\omega_{x}, \omega_{y}, \theta\right)=\frac{-\alpha}{2 \pi D_{11} i W_{\frac{-4\left(\alpha+D_{22} \rho^{2}\right)}{D_{11}}-\frac{\kappa_{0}^{2}}{D_{11}^{2}, i} \frac{2 \rho}{D_{11}}}} \\
& \times\left[e^{\frac{\kappa_{0} \theta}{2 D_{11}}} \mathrm{me}_{\nu}\left(\frac{\varphi}{2}, i \frac{2 \rho}{D_{11}}\right) \mathrm{me}_{-\nu}\left(\frac{\varphi-\theta}{2}, i \frac{2 \rho}{D_{11}}\right) \mathrm{u}(\theta)\right. \\
& \left.+e^{\frac{\kappa_{0} \theta}{2 D_{11}}} \mathrm{me}_{-\nu}\left(\frac{\varphi}{2}, i \frac{2 \rho}{D_{11}}\right) \mathrm{me}_{\nu}\left(\frac{\varphi-\theta}{2}, i \frac{2 \rho}{D_{11}}\right) \mathrm{u}(-\theta)\right]
\end{aligned}
$$

with Floquet exponent $\nu=\nu\left(-4 \frac{\left(\alpha+D_{22} \rho^{2}\right)}{D_{11}}-\frac{\kappa_{0}^{2}}{D_{11}^{2}}, i \frac{2 \rho}{D_{11}}\right)$.

Proof. As we generalize the results in Lemma 4.4 and Theorem 4.5, we follow the same construction. First we notice that the linear space of the solution of

$$
\left(\left(\partial_{\theta}\right)^{2}+k \partial_{\theta}-i R \cos (\varphi-\theta)-\beta\right) G(\theta)=0, \quad R \in \mathbb{R}, k \in \mathbb{R}, \beta>0,
$$

is spanned by the two Floquet solutions:

$$
\left\{e^{\frac{k \theta}{2}} \operatorname{me}_{\nu}\left(\frac{\varphi-\theta}{2}, 2 i R\right), e^{\frac{k \theta}{2}} \mathrm{me}_{-\nu}\left(\frac{\varphi-\theta}{2}, 2 i R\right)\right\}
$$

where $\nu=\nu\left(-k^{2}-4 \beta, 2 i R\right)$ equals the Floquet exponent. Now again we search for the unique direction within that span that vanishes at $\theta \rightarrow \infty$. Since $\nu\left(-k^{2}-4 \beta, 2 i R\right)$ is positively imaginary, the only candidate is $\theta \mapsto e^{\frac{k \theta}{2}} \mathrm{me}_{-\nu}\left(\frac{\varphi-\theta}{2}, 2 i R\right)$. The question remains whether $\operatorname{me}_{\nu}\left(\frac{\varphi-\theta}{2}, 2 i R\right)$ dominates the exploding factor $e^{\frac{k \theta}{2}}$ as $\theta \rightarrow+\infty$. This only holds if

$$
\frac{k}{2}+i \frac{\nu\left(-k^{2}-4 \beta, 2 i R\right)}{2}<0
$$

which indeed turns out to be the case

$$
\frac{k}{2}+i \frac{\nu\left(-k^{2}-4 \beta, 2 i R\right)}{2}<\frac{k}{2}+i \frac{\nu\left(-k^{2}-4 \beta, 0\right)}{2}=\frac{k}{2}+i \frac{\sqrt{-k^{2}-4 \beta}}{2}=\frac{k}{2}-\frac{\sqrt{k^{2}+4 \beta}}{2}<0 .
$$

Similarly, all of the solutions of (4.69) that converge for $\theta \rightarrow-\infty$ are spanned by $e^{\frac{k \theta}{2}} \mathrm{me}_{-\nu}\left(\frac{\varphi-\theta}{2}, 2 i R\right)$. Again we find the Green's function by continuous connection of two solutions for $\theta<0$ and $\theta>0$, where we put $k=\frac{\kappa_{0}}{D_{11}}, \beta=\frac{\alpha+D_{22} \rho^{2}}{D_{11}}$ and $R=\frac{\rho}{D_{11}}$. Then we calculate the scaling factor $\lambda$; recall the proof of Lemma 4.4. Analogously to (4.33) we get

$$
\begin{aligned}
& \quad \frac{\alpha}{2 \pi D_{11}} \delta_{0}=\lambda\left[-\frac{\kappa_{0}}{2} \mathrm{me}_{-\nu}\left(\frac{\varphi}{2}, i \frac{2 \rho}{D_{11}}\right) \mathrm{me}_{\nu}\left(\frac{\varphi}{2}, i \frac{2 \rho}{D_{11}}\right)+\frac{\kappa_{0}}{2} \mathrm{me}_{-\nu}\left(\frac{\varphi}{2}, i \frac{2 \rho}{D_{11}}\right) \mathrm{me}_{\nu}\left(\frac{\varphi}{2}, i \frac{2 \rho}{D_{11}}\right)\right. \\
& \left.\quad-\frac{1}{2}\left(\mathrm{me}_{\nu}\left(\frac{\varphi}{2}, 2 i R\right) \mathrm{me}_{-\nu}^{\prime}\left(\frac{\varphi}{2}, 2 i R\right)-\mathrm{me}_{-\nu}\left(\frac{\varphi}{2}, 2 i R\right) \mathrm{me}_{\nu}^{\prime}\left(\frac{\varphi}{2}, 2 i R\right)\right)\right] \delta_{0} \\
& \text { so }-i \lambda W_{-4 \beta^{\prime}, 2 i R} \delta_{0}=\frac{\alpha D_{11}}{2 \pi} \delta_{0}, \text { so } \lambda=\frac{-\alpha D_{11}}{2 \pi i W_{-4 \beta^{\prime}, 2 i R}}, \text { with } \beta^{\prime}=\frac{-4\left(\alpha+D_{22} \rho^{2}\right)}{D_{11}}-\frac{\kappa_{0}^{2}}{D_{11}^{2}} .
\end{aligned}
$$

See the lower part of Figures 5, 6 and 7 for plots of the Green's function for the case $\kappa_{0}=0.1,0.2$ and $D_{22}=D_{33}=0$.

5. Numerical scheme for the general case $a_{i}>0, D_{i i}>0$ and its relation to the exact analytic solutions. The following numerical scheme is a generalization of the numerical scheme proposed by Jonas August for the direction process ([5]). As explained in [13], this scheme is preferable over finite element methods. The reason for this is the non-commutativity of the Euclidean motion group in combination with the fact 
that the generator contains both a convection and diffusion part. ${ }^{22}$ Another advantage of this scheme over others, such as the algorithm by Zweck et al. [51], is that it is directly related to the exact analytic solutions presented in this paper, as we will show explicitly for the direction process case $a_{2}=1, a_{1}=a_{3}=0, D_{22}=D_{33}=0$.

The goal is to obtain a numerical approximation of the exact solution of

$$
\alpha(\alpha I-A)^{-1} U=W, U \in \mathbb{L}_{2}(G),
$$

where the generator $A$ is given in the general form (3.6) without further assumptions on the parameters $a_{i}>0, D_{i i}>0$. As explained earlier, the solution is given by a $G$-convolution with the corresponding Green's function. After explaining this scheme, we focus on the direction process case to show the connection with the exact solution (4.1). We give the explicit inverse of the matrix to be inverted within that scheme and we provide the full system of eigenfunctions of this matrix, which directly correspond to the exact solution (4.1). Although not considered here, we notice that exactly the same can be done for the other cases where we provide exact solutions. First we write

$$
\begin{aligned}
& \mathcal{F}\left[W\left(\cdot, e^{i \theta}\right)\right](\boldsymbol{\omega})=\hat{W}\left(\boldsymbol{\omega}, e^{i \theta}\right)=\sum_{l=-\infty}^{\infty} \hat{W}^{l}(\boldsymbol{\omega}) e^{i l \theta}, \\
& \mathcal{F}\left[U\left(\cdot, e^{i \theta}\right)\right](\boldsymbol{\omega})=\hat{U}\left(\boldsymbol{\omega}, e^{i \theta}\right)=\sum_{l=-\infty}^{\infty} \hat{U}^{l}(\boldsymbol{\omega}) e^{i l \theta} .
\end{aligned}
$$

Then by substituting (5.2) into (5.1) we obtain the following 4-fold recursion:

$$
\begin{aligned}
& \left(\alpha+l^{2} D_{11}+i a_{1} l+\frac{\rho^{2}}{2}\left(D_{22}+D_{33}\right)\right) \hat{W}^{l}(\boldsymbol{\omega})+\frac{a_{2}\left(i \omega_{x}+\omega_{y}\right)+a_{3}\left(i \omega_{y}-\omega_{x}\right)}{2} \hat{W}^{l-1}(\boldsymbol{\omega}) \\
& +\frac{a_{2}\left(i \omega_{x}-\omega_{y}\right)+a_{3}\left(i \omega_{y}+\omega_{x}\right)}{2} \hat{W}^{l+1}(\boldsymbol{\omega})-\frac{D_{22}\left(i \omega_{x}+\omega_{y}\right)^{2}+D_{33}\left(i \omega_{y}-\omega_{x}\right)^{2}}{4} \hat{W}^{l-2}(\boldsymbol{\omega}) \\
& -\frac{D_{22}\left(i \omega_{x}-\omega_{y}\right)^{2}+D_{33}\left(i \omega_{y}+\omega_{x}\right)^{2}}{4} \hat{W}^{l+2}(\boldsymbol{\omega})=\alpha \hat{U}^{l}(\boldsymbol{\omega})
\end{aligned}
$$

which can be rewritten in polar coordinates

$$
\begin{aligned}
& \left(\alpha+i l a_{1}+D_{11} l^{2}+\frac{\rho^{2}}{2}\left(D_{22}+D_{33}\right)\right) \tilde{W}^{l}(\rho)+\frac{\rho}{2}\left(i a_{2}-a_{3}\right) \tilde{W}^{l-1}(\rho) \\
& +\frac{\rho}{2}\left(i a_{2}+a_{3}\right) \tilde{W}^{l+1}(\rho)+\frac{\rho^{2}}{4}\left(D_{22}-D_{33}\right)\left(\tilde{W}^{l+2}(\rho)+\tilde{W}^{l-2}(\rho)\right)=\alpha \tilde{U}^{l}(\rho)
\end{aligned}
$$

for all $l=0,1,2, \ldots$ with $\tilde{W}^{l}(\rho)=e^{i l \varphi} \hat{W}^{l}(\boldsymbol{\omega})$ and $\tilde{U}^{l}(\rho)=e^{i l \varphi} \hat{U}^{l}(\boldsymbol{\omega})$, with $\boldsymbol{\omega}=$ $(\rho \cos \varphi, \rho \sin \varphi)$. Equation (5.4) can easily be written in matrix form, where a 5-band matrix must be inverted. For explicit representation of this 5-band matrix where the spatial Fourier transform in (5.2) is replaced by the discrete Fourier transform, we refer to $[13$, p. 230]. Here we stick to a Fourier series on $\mathbb{T}$, the continuous Fourier transform on $\mathbb{R}^{2}$, and the truncation of the series at $N \in \mathbb{N}$ yields the $(2 N+1) \times(2 N+1)$ matrix

\footnotetext{
${ }^{22}$ If one insists on using a finite element method, a sensible approach is to alternate the noncommuting diffusion and convection parts with very small step sizes such that the CBH formula can be numerically truncated, $e^{s(\text { Diff }+ \text { Conv })} \approx e^{s \text { Diff }} e^{s \text { Conv }}$, which is the idea behind Zweck's algorithm [51].
} 
equation:

$$
\left(\begin{array}{ccccccc}
p-N & q+t & r & 0 & 0 & 0 & 0 \\
q-t & p_{-N+1} & q+t & r & 0 & 0 & 0 \\
r & \ddots & \ddots & \ddots & r & 0 & 0 \\
0 & \ddots & q-t & p_{0} & q+t & r & 0 \\
0 & 0 & r & \ddots & \ddots & \ddots & r \\
0 & 0 & 0 & r & q-t & p_{N-1} & q+t \\
0 & 0 & 0 & 0 & r & q-t & p_{N}
\end{array}\right)\left(\begin{array}{c}
\tilde{W}^{-N}(\rho) \\
\tilde{W}^{-N+1}(\rho) \\
\vdots \\
\tilde{W}^{0}(\rho) \\
\vdots \\
\tilde{W}^{N-1}(\rho) \\
\tilde{W}^{N}(\rho)
\end{array}\right)=\frac{4 \alpha}{D_{11}}\left(\begin{array}{c}
\tilde{U}^{-N}(\rho) \\
\tilde{U}^{-N+1}(\rho) \\
\vdots \\
\tilde{U}^{0}(\rho) \\
\vdots \\
\tilde{U}^{N-1}(\rho) \\
\tilde{U}^{N}(\rho)
\end{array}\right)
$$

where $p_{l}=(2 l)^{2}+\frac{4 \alpha+2 \rho^{2}\left(D_{22}+D_{33}\right)+4 i a_{1} l}{D_{11}}, r=\frac{\rho^{2}\left(D_{22}-D_{33}\right)}{D_{11}}, q=\frac{2 \rho a_{2} i}{D_{11}}$ and $t=\frac{2 a_{3} \rho}{D_{11}}$

For the sake of simplicity and illustration we will only consider the direction process case with $a_{2}=1, a_{1}=a_{3}=0, D_{22}=D_{33}=0$ (although we stress that the other cases can be treated similarly). In this case we have $p_{l}=(2 l)^{2}+\frac{4 \alpha}{D_{11}}, r=0, q=\frac{2 \rho i}{D_{11}}$ and $t=0$ and thereby the recursion (5.3) is 2 -fold and the equation requires the inversion of a 3-band matrix, the complete eigensystem (for $N \rightarrow \infty$ ) of which is given by

$$
\left\{\begin{array}{l}
\mathbf{v}_{l}=\left\{c_{2 l}^{2 n}(q)\right\}_{n=-N}^{N}, \quad N \rightarrow \infty, \\
\lambda_{l}=a_{2 l}(q)+\frac{4 \alpha}{D_{11}}, \quad l \in \mathbb{Z},
\end{array}\right.
$$

where $a_{2 l}(q)$ and $c_{2 l}^{2 n}(q)$ are, respectively, the Mathieu characteristic and Mathieu coefficients; recall (4.13) which can considered as an eigenvalue problem of a 3-band matrix. The eigenvectors form a bi-orthogonal basis in $\ell_{2}(\mathbb{Z})$ and the basis transforms between the orthogonal standard basis $\epsilon=\left\{\mathbf{e}_{l}\right\}_{l \in \mathbb{Z}}$ in $\ell_{2}(\mathbb{Z})$ (which corresponds to $\left.\left\{\theta \mapsto e^{i l \theta}\right\}_{l \in \mathbb{Z}} \in \mathbb{L}_{2}([0,2 \pi))\right)$ and the bi-orthogonal basis of eigenvectors $\beta=\left\{\mathbf{v}_{l}\right\}_{l \in \mathbb{Z}}$ (which corresponds to $\left.\left\{\theta \mapsto \operatorname{me}_{2 n}\left(\frac{\varphi-\theta}{2}, q\right)\right\}_{l \in \mathbb{Z}}\right)$ is

$$
S_{\alpha}^{\epsilon}=\frac{1}{\sqrt{2 \pi}}\left(\begin{array}{lllllll}
\mathbf{v}_{1} & \mid & \mathbf{v}_{2} & \mid & \mathbf{v}_{3} & \mid & \ldots
\end{array}\right) \quad S_{\epsilon}^{\alpha}=\frac{1}{\sqrt{2 \pi}}\left(S_{\alpha}^{\epsilon}\right)^{T},
$$

where we stress that the transpose does not include a conjugation, so $S_{\epsilon}^{\alpha}=\left(S_{\alpha}^{\epsilon}\right)^{-1}=$ $\overline{\left(S_{\alpha}^{\epsilon}\right)^{\dagger}}$. To this end we notice that $\sum_{l=-\infty}^{\infty} c_{2 l}^{2 r}(q) c_{2 l}^{2 s}(q)=\delta^{r s}$, which directly follows from the bi-orthogonality of the corresponding Mathieu functions in $\mathbb{L}_{2}([0, \pi])$. Now

$$
\hat{\mathbf{w}}=\frac{4 \alpha}{D_{11}}\left(S_{\alpha}^{\epsilon} \Lambda S_{\epsilon}^{\alpha}\right)^{-1} \mathbf{u}=\frac{4 \alpha}{D_{11}}\left(S_{\alpha}^{\epsilon} \Lambda^{-1} S_{\epsilon}^{\alpha}\right) \hat{\mathbf{u}}
$$

where $\Lambda=\operatorname{diag}\left(\left\{\lambda_{l}\right\}\right)$ and where $\hat{\mathbf{w}}=\left\{\hat{W}^{l}\right\}_{l \in \mathbb{Z}}$ and $\hat{\mathbf{u}}=\left\{\hat{U}^{l}\right\}_{l \in \mathbb{Z}}$, so the general solution of $(5.5)$ is given by

$$
\tilde{W}^{l}(\rho)=\frac{1}{2 \pi} \frac{4 \alpha}{D_{11}}\left(S_{\epsilon}^{\alpha}\right)_{m}^{l} \delta_{n}^{m} \frac{1}{a_{2 n}(q)+\frac{4 \alpha}{D_{11}}}\left(S_{\alpha}^{\epsilon}\right)_{p}^{n} \tilde{U}^{p}(\rho)=\frac{\alpha}{2 \pi} \sum_{n \in \mathbb{Z}} \sum_{p \in \mathbb{Z}} \frac{c_{2 l}^{2 n}(q) c_{2 p}^{2 n}(q)}{\lambda_{n}(\rho)} \tilde{U}^{p}(\rho),
$$

with $\lambda_{n}(\rho)=\alpha+\frac{a_{2 n}\left(\frac{2 \rho i}{D_{11}}\right) D_{11}}{4}$ where we used the summation convention for double indices. As a result we have

$$
\begin{aligned}
& \hat{W}(\boldsymbol{\omega}, \theta)=\sum_{l \in \mathbb{Z}} \hat{W}^{l}(\boldsymbol{\omega}) e^{i l \theta}=\sum_{l \in \mathbb{Z}} e^{i l(\theta-\varphi)} \tilde{W}^{l}(\rho) \\
& =\frac{\alpha}{2 \pi} \lim _{N \rightarrow \infty} \sum_{n=-N}^{N}\left(\sum_{l=-N}^{N} \frac{c_{2 l}^{2 n}(q) e^{i l(\theta-\varphi)}}{\lambda_{n}(\rho)}\right)\left(\sum_{p=-N}^{N} c_{2 p}^{2 n}(q) e^{i p \varphi} \hat{U}^{p}(\rho)\right) \\
& =\frac{\alpha}{2 \pi} \sum_{n \in \mathbb{Z}} \frac{\operatorname{me}_{2 n}\left(\frac{\theta-\varphi}{2}, q\right)}{\lambda_{n}(\rho)} \sum_{p \in \mathbb{Z}} c_{2 p}^{2 n}(q) e^{i p \varphi} \hat{U}^{p}(\rho),
\end{aligned}
$$



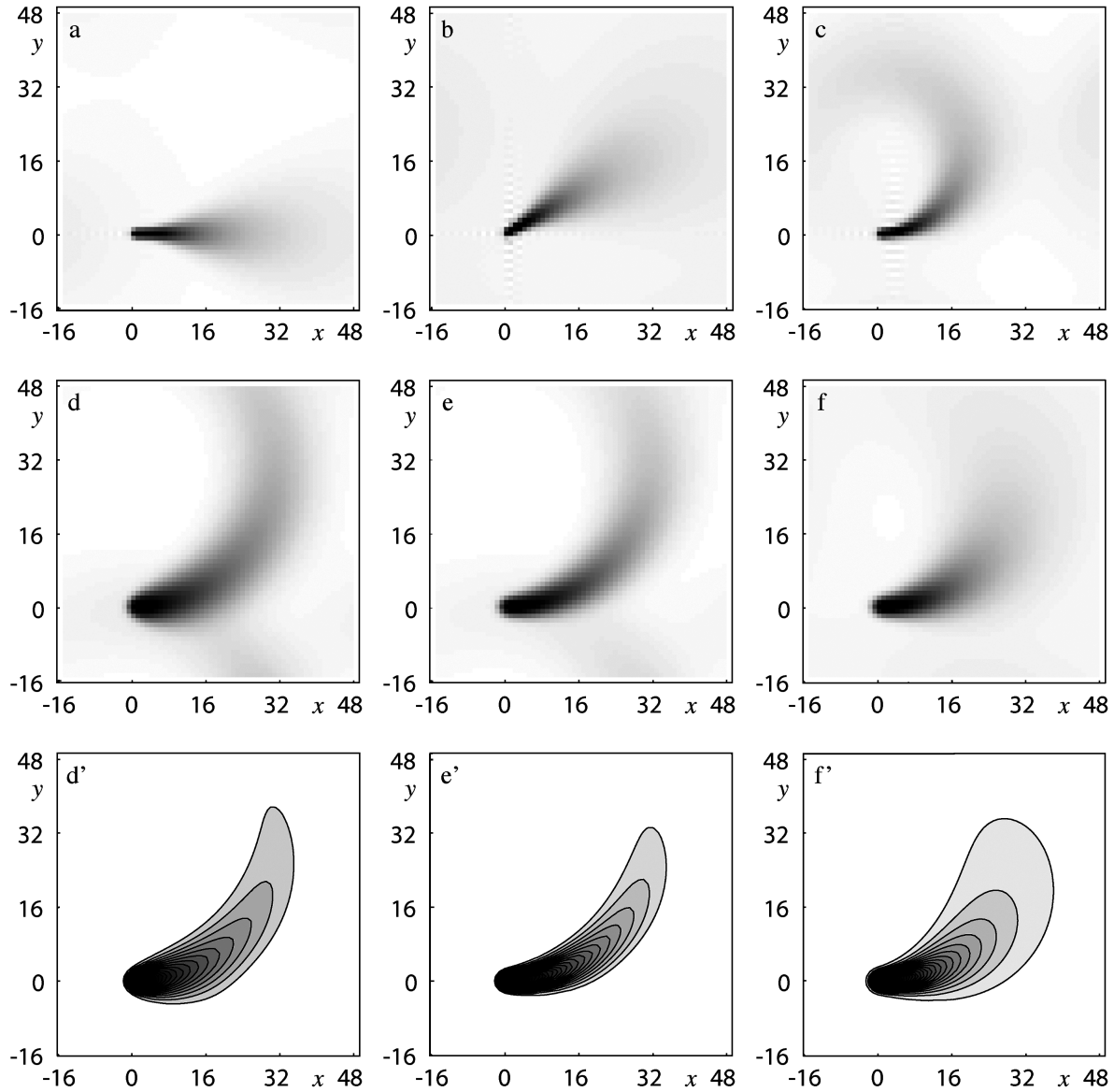

FIG. 10. Left-invariant evolutions on the Euclidean motion group yields graphical sketching for image analysis. Computation of the $x y$-marginals (integration over $\theta$ from 0 to $2 \pi$ ) of the Green's function $S_{\alpha, D_{11}, D_{22}, D_{33}}^{a_{1}, a_{2}, a_{3}, x_{0}, y_{0}, \theta_{0}}=e^{s \Delta} \alpha(-A+\alpha I)^{-1} \delta_{\left(x_{0}, y_{0}, \theta_{0}\right)}$, where $A$ is the generator in its general form (3.6) for different parameter settings. We used the fast Fourier transform on a $64 \times 64 \times 64$ grid in the algorithm of Section 5 (we put $s=\frac{\sigma^{2}}{2}>0$ (4.35) with $\sigma>0$ in the order of magnitude of 1 pixel). Respective (from (a) to (f)) parameter settings are $\left(\alpha ; a_{1}, a_{2}, a_{3} ; D_{11}, D_{22}, D_{33}\right)=$ $\left(\frac{1}{64} ; 0,1,0 ;\left(\frac{2 \pi}{128}\right)^{2}, 0,0\right), \quad\left(\frac{1}{64} ; 0,1,0 ;\left(\frac{2 \pi}{128}\right)^{2}, 0,0\right), \quad\left(\frac{1}{64} ; 0.1,1,0 ;\left(\frac{2 \pi}{128}\right)^{2}, 0,0\right)$, $\left(\frac{1}{40} ; \frac{1}{32}, 1,0 ; 0,0.1,0.4\right),\left(\frac{1}{40} ; \frac{1}{32}, 1,0 ; 0,0.4,0.1\right),\left(\frac{1}{40} ; \frac{1}{32}, 1,0 ;\left(\frac{2 \pi}{128}\right)^{2}, 0.4,0.1\right)$. In all cases the initial condition is $U=\delta_{e}$, except for case (b) where $U=\delta_{0}^{x} \otimes \delta_{0}^{y} \otimes \delta_{\theta_{0}}^{\theta}$ with $\theta_{0}=\pi / 6$. The top row illustrates the left-invariance of the evolution equations; the bottom two rows (the last row is a contour plot of the same Green's functions (d,e,f)) show spatial and angular diffusion. Figures (e) and (f) reveal the non-commutativity of angular and anisotropic spatial diffusion. 
with $q=\frac{2 \rho i}{D_{11}}$ and $\boldsymbol{\omega}=(\rho \cos \varphi, \rho \sin \varphi)$. Now if we put $\hat{U}^{p}=\frac{1}{2 \pi}$ for all $p \in \mathbb{Z}$ (i.e. $\left.U=\delta_{e}\right)$, we get the impulse response, i.e. the Green's function

$$
\hat{S}_{\alpha, D_{11}}(\boldsymbol{\omega}, \theta)=\frac{\alpha}{(2 \pi)^{2}} \sum_{n \in \mathbb{Z}} \frac{\mathrm{me}_{2 n}\left(\frac{\varphi}{2}, \frac{2 \rho i}{D_{11}}\right) \mathrm{me}_{2 n}\left(\frac{\theta-\varphi}{2}, \frac{2 \rho i}{D_{11}}\right)}{\lambda_{n}(\rho)},
$$

which is indeed the exact solution (4.24) in Theorem 4.1, where we recall that $W=$ $S_{\alpha, D_{11}} *_{G} U$. The advantage of (5.6) is that it is efficient and does not require orientation interpolations. See Figure 10.

Acknowledgements. This work has been greatly inspired by the work of Prof. D. Mumford (Department of Mathematics, Brown University) on elastics in computer vision ([40]). The theory and solutions we provide in this article were both motivated by an open problem posed by Prof. D. Mumford within [40] and by several explicit image analysis applications we studied in the biomedical image analysis group ${ }^{23}$ of Prof. B. ter Haar-Romeny and Dr. Ir. L. M. J. Florack at the biomedical engineering department of Eindhoven University of Technology.

The authors wish to thank Dr. A. F. M. ter Elst (Department of Mathematics, University of Auckland) for pointing us to the Euclidean motion group structure within the direction process and Prof. J. de Graaf (Department of Mathematics, Eindhoven University of Technology) for reminders on general techniques for deriving Green's functions and thanks to Prof. D. Mumford for his suggestions, after carefully reading this article.

Appendix A. Simple expressions for the exact solutions in terms of the Fourier transform on the Euclidean motion group. In this section we will use the Fourier transform on the Euclidean motion group, rather than the Fourier transform on $\mathbb{R}^{2}$ as was done in Theorem 4.1 and Theorem 4.10, to get explicit expressions for the Green's functions on the Euclidean motion group. Although these expressions are similar to the ones we previously derived, this approach provides further insight into the underlying group structure and moreover it provides a shortcut to Mathieu's equation. For the sake of illustration we restrict ourselves to the generalized direction process as discussed in Subsection 4.6. The same can be achieved for the general case $\left\{a_{i}\right\}_{i=1}^{3} \in \mathbb{R}^{3}$, $\left\{D_{i i}\right\}_{i=1}^{3} \in\left(\mathbb{R}^{+}\right)^{3}$.

According to [43] all unitary irreducible representations of the 2D Euclidean motion group $G=\mathbb{R}^{2} \rtimes \mathbb{T}$ are defined on $\mathbb{L}_{2}\left(S_{1}\right)$ and they are given by

$$
\mathcal{V}_{g}^{p} f(\mathbf{y})=e^{-i p(\mathbf{x}, \mathbf{y})} f\left(A^{-1} \mathbf{y}\right), \quad f \in \mathbb{L}_{2}\left(S_{1}\right), g=\left(\mathbf{x}, e^{i \theta}\right) \in G, p>0,
$$

for almost every $\mathbf{y}=(\cos \phi, \sin \phi) \in S_{1}$. Notice that each such unitary representation can be identified with its matrix elements

$$
\left(\eta_{n}, \mathcal{V}_{g}^{p} \eta_{m}\right)=V_{m n}^{p}(g)=i^{n-m} e^{-i(n \theta+(m-n) \phi)} J_{n-m}(\rho a)
$$

with respect to the orthonormal basis $\left\{\eta_{n}\right\}_{n \in \mathbb{Z}}:=\left\{\theta \mapsto e^{i n \theta}\right\}_{n \in \mathbb{Z}}$. Consequently, the Fourier transform on the Euclidean motion group $\mathcal{F}_{G}: \mathbb{L}_{2}(G) \rightarrow \mathbb{L}_{2}\left(T_{2}\left(\mathbb{L}_{2}\left(S_{1}\right)\right)\right.$, pd $\left.p\right)$,

\footnotetext{
${ }^{23}$ All Green's functions within this article are included in the Mathematica package MathVisionTools, available (for academic purposes only) on the web at http://www.mathvisiontools.net
} 
where $T_{2}=\left\{A \in B\left(\mathbb{L}_{2}\left(S_{1}\right)\right) \mid\|A\|_{2}^{2}=\operatorname{trace}\left(\mathcal{A}^{*} \mathcal{A}\right)<\infty\right\}$, is given by

$$
\left[\mathcal{F}_{G} f\right](p)=\int_{G} f(g) V_{g^{-1}}^{p} \mathrm{~d} \mu_{G}(g),
$$

and its inverse is almost everywhere given by $\left[\mathcal{F}_{G}^{-1} \hat{f}\right](g)=\int_{0}^{\infty} \operatorname{trace}\left\{\hat{f}(p) V_{g}^{p}\right\} p \mathrm{~d} p$. This Fourier transform is unitary as by Parceval's identity we have

$$
\|f\|_{\mathbb{L}_{2}(G)}^{2}=\int_{0}^{\infty}\left\|\mathcal{F}_{G} f(p)\right\|_{2}^{2} p \mathrm{~d} p=\left\|\mathcal{F}_{G} f\right\|_{\mathbb{L}_{2}\left(T_{2}\left(\mathbb{L}_{2}\left(S_{1}\right)\right), p \mathrm{~d} p\right)}^{2} .
$$

Now it is straightforward (use left-invariance of the Haar measure and switch the order of integration) to show that $\mathcal{F}_{G}\left(f_{1} *_{G} f_{2}\right)=\mathcal{F}_{G} f_{1} \mathcal{F}_{G} f_{2}$. As a result the solution of the generalized direction process $W=(A-\alpha I)^{-1} U$ is given by

$$
W=\mathcal{F}_{G}^{-1}\left[\mathcal{F}_{G} S_{a_{1}, a_{2}, D_{11}, D_{22}, \alpha} \mathcal{F}_{G} U\right]
$$

where $S_{a_{1}, a_{2}, D_{11}, D_{22}, \alpha}$ equals the Green's function. So (A.2) together with (A.1) provide a simple alternative (but, as we will see, similar) algorithm to the algorithm discussed in Section 5 if we are able to compute the matrix coefficients of the Fourier transform of the Green's function. We shall need the following lemma.

Lemma A.1. For all $p>0$ and all $h \in G$ and all $f \in \mathbb{L}_{2}(G)$, we have

$$
\left[\mathcal{F}_{G} \mathcal{R}_{h} f\right](p)=V_{h}^{p}\left[\mathcal{F}_{G} f\right](p) .
$$

Consequently we have $\mathcal{F}_{G}[\mathrm{~d} \mathcal{R}(A) f](p)=\mathrm{d} V^{p}(A)\left[\mathcal{F}_{G} f\right](p)$ for all $A \in T_{e}(G), f \in \mathbb{L}_{2}(G)$ and $p>0$. So in particular

$$
\mathrm{d} V^{p}\left(A_{1}\right)=-i p \cos \phi, \quad \mathrm{d} V^{p}\left(A_{2}\right)=-i p \sin \phi, \quad \mathrm{d} V^{p}\left(A_{3}\right)=\partial_{\phi} .
$$

Proof. With respect to the first equality we notice that

$$
\left[\mathcal{F}_{G} \mathcal{R}_{h} f\right](p)=\int_{G} f(g h) V_{g^{-1}}^{p} \mathrm{~d} \mu_{G}(g)=\int_{G} f\left(g^{\prime}\right) V_{h}^{p} V_{\left(g^{\prime}\right)^{-1}}^{p} \mathrm{~d} \mu_{G}\left(g^{\prime}\right)=V_{h}^{p}\left[\mathcal{F}_{G} f\right](p) .
$$

Now the second equality follows by the first as we have

$$
\mathcal{F}_{G}\left[\lim _{t \rightarrow 0} \frac{\mathcal{R}_{e^{t A}-I}}{t} f\right](p)=\lim _{t \rightarrow 0}\left(\frac{V_{e^{t A}}^{p}-I}{t}\right)\left[\mathcal{F}_{G} f\right](p)=\mathrm{d} V^{p}(A)\left[\mathcal{F}_{G} f\right](p) .
$$

Now the special cases (A.3) follow by direct computation.

Consequently, by applying the Fourier transform on both sides of the resolvent equation directly leads to Mathieu's equation:

Theorem A.2. The Fourier transform of the Green's function $\mathcal{F}_{G} S_{a_{1}, a_{2}, D_{11}, D_{22}, \alpha}$ satisfies

$$
\left(a_{1} \partial_{\phi}-i a_{2} p \cos \phi+D_{22} p^{2}+D_{11}\left(\partial_{\phi}\right)^{2}+\alpha\right)\left[\mathcal{F}_{G} S_{a_{1}, a_{2}, D_{11}, D_{22}, \alpha}\right](p)=I,
$$

as with (4.9), and thereby the matrix representation of the operator $\mathcal{F}_{G} S_{a_{1}, a_{2}, D_{11}, D_{22}, \alpha}$ yields a similar matrix as in equation (5.5), where the role of $(p, \phi)$ and $(\rho, \theta-\varphi)$ are switched.

However, the results in this appendix allow the use of well-known fast implementations of the Fourier transform on $\mathbb{R}^{2} \rtimes \mathbb{T}$ for computation of the Green's functions and the corresponding $G$-convolution (4.4) on orientation scores. 


\section{RefEREnCES}

[1] M. Abramowitz and I. A. Stegun, editors. Handbook of Mathematical Functions with Formulas, Graphs, and Mathematical Tables. Dover Publications, Inc., New York, 1965. Originally published by the National Bureau of Standards in 1964.

[2] M. A. van Almsick. Context Models of Lines and Contours PhD thesis, Eindhoven University of Technology, Department of Biomedical Engineering, Eindhoven, The Netherlands, 2007.

[3] J.P. Antoine. Directional wavelets revisited: Cauchy wavelets and symmetry detection in patterns. Applied and Computational Harmonic Analysis, 6:314-345, 1999. MR1685408 (2000b:42025)

[4] N. Aronszajn. Theory of reproducing kernels, Trans. A.M.S., vol. 68, pp. 337-404, 1950. MR0051437 $(14: 479 \mathrm{c})$

[5] J. August. The Curve Indicator Random Field. PhD thesis, Yale University, 2001.

[6] J. August and S.W. Zucker. The curve indicator random field and markov processes. IEEE-PAMI, Pattern Recognition and Machine Intelligence, 25, 2003. Number 4.

[7] G. Blanch and D. S. Clemm. The double points of Mathieu's equation. Math.Comp., 23:97-108, 1969. MR0239727 (39:1084)

[8] W.H. Bosking, Y. Zhang, B. Schofield, and D. Fitzpatrick. Orientation selectivity and the arrangement of horizontal connections in tree shrew striate cortex. The Journal of Neuroscience, 17(6):2112-2127, March 1997.

[9] G. Citti and A. Sarti. A cortical based model of perceptional completion in the roto-translation space. Journal of Mathematical Imaging and Vision, 24:307-326, 2006.

[10] M. Duits. A functional Hilbert space approach to frame transforms and wavelet transforms. September 2004. Master thesis in Applied Analysis. Dep. Mathematics and Computer Science, Eindhoven University of Technology

[11] M. Duits and R. Duits. Unitary wavelet transforms based on reducible representations of the affine group. In preparation.

[12] R. Duits, E.M. Franken and M. van Almsick. Contour Enhancement via linear and non-linear evolution equations on the Euclidean Motion Group. In preparation.

[13] R. Duits. Perceptual Organization in Image Analysis. PhD thesis, Eindhoven University of Technology, Department of Biomedical Engineering, The Netherlands, 2005. A digital version is available at URL: http:// www.bmi2.bmt.tue.nl/Image-Analysis/People/RDuits/THESISRDUITS.pdf.

[14] R. Duits and M. van Almsick, The explicit solutions of the left-invariant evolution equations on the Euclidean motion group. Technical Report CASA 05-43, Department of Mathematics and Computer science, Eindhoven University of Technolgy, The Netherlands, December 2005. A digital version is available on the web at URL: http://yp.bmt.tue.nl/pdfs/6321.pdf.

[15] R. Duits, M. Felsberg, G. Granlund, and B.M. ter Haar Romeny. Image analysis and reconstruction using a wavelet transform constructed from a reducible representation of the euclidean motion group. International Journal of Computer Vision. Accepted for publication. To appear in Volume 72, issue 1, April 2007.

[16] R. Duits, M. Duits, M. van Almsick and B.M. ter Haar Romeny. Invertible Orientation Scores as an Application of Generalized Wavelet Theory. Image Processing, Analysis, Recognition, and Understanding. Volume 17, Nr. 1: pp. 42-75, 2007.

[17] R. Duits, L.M.J. Florack, J. de Graaf, and B. ter Haar Romeny. On the axioms of scale space theory. Journal of Mathematical Imaging and Vision, 20:267-298, May 2004. MR2060148 (2005k:94005)

[18] R. Duits, M. van Almsick, M. Duits, E. Franken, and L.M.J. Florack. Image processing via shifttwist invariant operations on orientation bundle functions. In Niemann Zhuralev et al. Geppener, Gurevich, editor, 7th International Conference on Pattern Recognition and Image Analysis: New Information Technologies, pages 193-196, St. Petersburg, October 2004.

[19] R. Duits, B. Burgeth. Scale Spaces on Lie groups. In the proceedings of SSVM 2007, 1st international conference on scale space and variational methods in computer vision, Lecture Notes on Computer Science, Springer-Verlag, 2007, p. 300-312, Ischia, Italy, June 2007.

[20] N. Dungey, A. F. M. ter Elst, and D. W. Robinson. Analysis on Lie groups with polynomial growth, volume 214. Birkhauser-Progress in Mathematics, Boston, 2003. MR2000440 (2004i:22010)

[21] M. Felsberg, P.-E. Forssén, and H. Scharr. Efficient robust smoothing of low-level signal features. Technical Report LiTH-ISY-R-2619, SE-581, 83 Linkoping, Sweden, August 2004. 
[22] M. Felsberg, P.-E. Forssén, and H. Scharr. Channel smoothing: Efficient robust smoothing of lowlevel signal features. IEEE Transactions on Pattern Analysis and Machine Intelligence, 2005. Accepted.

[23] G. Floquet. Sur les équations différentielles linéaires à coefficients périodiques. Ann. École Norm. Sup., 12(47), 1883.

[24] P.-E. Forssén and G. H. Granlund. Sparse feature maps in a scale hierarchy. In G. Sommer and Y.Y. Zeevi, editors, Proc. Int. Workshop on Algebraic Frames for the Perception-Action Cycle, volume 1888 of Lecture Notes in Computer Science, Kiel, Germany, September 2000. Springer, Heidelberg.

[25] P.E. Forssen. Low and Medium Level Vision using Channel Representations. PhD thesis, Linkoping University, Dept. EE, Linkoping, Sweden, March 2004.

[26] E. Franken, M. van Almsick, P. Rongen, L.M.J. Florack and B.M. ter Haar Romeny. An Efficient Method for Tensor Voting using Steerable Filters. Proceedings European Congress on Computer Vision 2006, 288-240, 2006.

[27] E. Franken, R. Duits and B.M. ter Haar Romeny. Nonlinear Diffusion on the Euclidean Motion Group. In the proc. of the 1 st international conference on scale space and variational methods in computer vision, Lecture Notes on Computer Science, Springer-Verlag, 2007, p. 461-472, Ischia, Italy, June 2007.

[28] H. Führ. Abstract Harmonic Analysis of Continuous Wavelet Transforms. Springer, HeidelbergNew York, 2005. MR2130226 (2006m:43003)

[29] A. Grossmann, J. Morlet, and T. Paul. Integral transforms associated to square integrable representations. J. Math. Phys., 26:2473-2479, 1985. MR803788 (86k:22013)

[30] W. Hebisch. Estimates on the semigroups generated by left-invariant operators on Lie groups. Journal fuer die Reine und Angewandte Mathematik, 423:1-45, 1992. MR1142482 (93d:22008)

[31] G. W. Hill. On the part of motion of the lunar perigee, which is a function of the mean motions of the sun and the moon. Acta Mathematica, 1, 1886.

[32] L. Hormander. Hypoellptic second order differential equations. Acta Mathematica, 119:147-171, 1968. MR0222474 (36:5526)

[33] C. Hunter and B. Guerrieri. The eigenvalues of Mathieu's equation and their branch points. Studies in Applied Mathematics, 64:113-141, 1981. MR608595 (82c:34030)

[34] P. E. T. Jorgensen. Representations of differential operators on a Lie group. Journal of Functional Analysis, 20:105-135, 1975. MR0383469 (52:4350)

[35] S. N. Kalitzin, B. M. ter Haar Romeny, and M. A. Viergever. Invertible apertured orientation filters in image analysis. Int. Journal of Computer Vision, 31(2/3):145-158, April 1999.

[36] T. S. Lee. Image representation using 2D gabor wavelets. IEEE-Transactions on Pattern Analysis and Machine Inteligence, 18(10):959-971, 1996.

[37] W. Magnus and S. Winkler. Hill's equation. Dover, New York, 1979. MR559928 (80k:34001)

[38] Gérard Medioni, Mi-Suen Lee, and Chi-Keung Tang. A Computational Framework for Segmentation and Grouping. Elsevier, Amsterdam.

[39] J. Meixner and F. W. Schaefke. Mathieusche Funktionen und Sphaeroidfunktionen. Springer-Verlag, Berlin-Gotingen-Heidelberg, 1954.

[40] D. Mumford. Elastica and computer vision. Algebraic Geometry and Its Applications. SpringerVerlag, pages 491-506, 1994. MR1272050 (95a:92026)

[41] B. Øksendahl. Stochastic differential equations: an introduction with applications. Springer, Berlin, 1998. MR1619188 (99c:60119)

[42] N. Petkov and M. Kruizinga. Computational models of visual measures specialized in the detection of periodic and aperiodic orientation visual stimuli and grating cells. Biological Cybernetics, 76:8396, 1997.

[43] Suigiura, M. Unitary representations and harmonic analysis. North-Holland, 2nd Edition, Mathematical Library, 44, Amsterdam, Kodansha, Tokyo, 1990. MR1049151 (91c:22028)

[44] K.K. Thornber and L.R. Williams. Analytic solution of stochastic completion fields. Biological Cybernetics, 75:141-151, 1996.

[45] K.K. Thornber and L.R. Williams. Characterizing the Distribution of Completion Shapes with Corners Using a Mixture of Random Processes. Pattern Recognition, 33:543-553, 2000.

[46] D. Y. Ts'0, R. D. Frostig, E. E. Lieke, and A. Grinvald. Functional organization of primate visual cortex revealed by high resolution optical imaging. Science, 249:417-20, 1990. 
[47] M. A. van Almsick, R. Duits, E. Franken, and B.M. ter Haar Romeny. From stochastic completion fields to tensor voting. In Proceedings DSSCC-workshop on Deep Structure Singularities and Computer Vision, pages 124-134, Maastricht, The Netherlands, June 9-10, 2005. Springer-Verlag.

[48] V.S. Varadarajan. Lie Groups, Lie-Algebras and Their Representations. Springer-Verlag, New York, Berlin, Heidelberg, Tokyo, 1984. MR746308 (85e:22001)

[49] H. Volkmer. On the growth of convergence radii for the eigenvalues of the Mathieu equation. Math. Nachr., 192:239-253, 1998. MR1626348 (2000a:34053)

[50] L. R. Williams and J.W. Zweck. A rotation and translation invariant saliency network. Biological Cybernetics, 88:2-10, 2003.

[51] J. Zweck and L. R. Williams. Euclidean group invariant computation of stochastic completion fields using shiftable-twistable functions. Journal of Mathematical Imaging and Vision, 21(2):135-154, 2004. MR2090129 (2005d:68131) 